\title{
Filamentary structure and Keplerian rotation in the high-mass star-forming region G35.03+0.35 imaged with ALMA ${ }^{\star}$
}

\author{
M. T. Beltrán ${ }^{1}$, Á. Sánchez-Monge ${ }^{1,2}$, R. Cesaroni ${ }^{1}$, M. S. N. Kumar ${ }^{3}$, D. Galli ${ }^{1}$, C. M. Walmsley ${ }^{1,4}$, S. Etoka ${ }^{5,6}$, \\ R. S. Furuya ${ }^{7}$, L. Moscadelli ${ }^{1}$, T. Stanke ${ }^{8}$, F. F. S. van der Tak ${ }^{9,10}$, S. Vig ${ }^{11}$, K.-S. Wang ${ }^{12,13}$, \\ H. Zinnecker ${ }^{14}$, D. Elia ${ }^{15}$, and E. Schisano ${ }^{16}$
}

1 INAF-Osservatorio Astrofisico di Arcetri, Largo E. Fermi 5, 50125 Firenze, Italy

2 I. Physikalisches Institut, Universität zu Köln, Zülpicher Str. 77, 50937 Köln, Germany

3 Centro de Astrofísica da Universidade do Porto, Rua das Estrelas, 4150-762 Porto, Portugal

${ }^{4}$ Dublin Institute for Advanced Studies (DIAS), 31 Fitzwilliam Place, 2 Dublin, Ireland

5 Jodrell Bank Centre for Astrophysics, School of Physics and Astronomy, University of Manchester, Manchester M13 9PL, UK

${ }^{6}$ Hamburger Sternwarte, Gojenbergsweg 112, 21029 Hamburg, Germany

7 The University of Tokushima, Minami Jousanjima-machi 1-1, Tokushima, 770-8502 Tokushima, Japan

8 ESO, Karl-Schwarzschild-Strasse 2, 85748 Garching bei München, Germany

9 SRON Netherlands Institute for Space Research, PO Box 800, 9700 AV Groningen, The Netherlands

10 Kapteyn Astronomical Institute, University of Groningen, 9700 AV Groningen, The Netherlands

11 Dept. of Earth and Space Science, Indian Institute of Space Science and Technology, Thiruvananthapuram, 695547 Kerala, India

12 Leiden Observatory, Leiden University, PO Box 9513, 2300 RA Leiden, The Netherlands

13 Academia Sinica, Institute of Astronomy and Astrophysics, PO Box 23-141, Taipei 10617, Taiwan, PR China

14 SOFIA Science Center, NASA Ames Research Center, Mailstop 232-12, Moffett Field, CA 94035, USA

15 Istituto di Astrofisica e Planetologia Spaziali - INAF, via Fosso del Cavaliere 100, 00133 Roma, Italy

16 Infrared Processing and Analysis Center, California Institute of Technology, Pasadena, CA 91125, USA

Received 18 April 2014 / Accepted 24 July 2014

\begin{abstract}
Context. Theoretical scenarios propose that high-mass stars are formed by disk-mediated accretion.

Aims. To test the theoretical predictions on the formation of massive stars, we wish to make a thorough study at high-angular resolution of the structure and kinematics of the dust and gas emission toward the high-mass star-forming region G35.03+0.35, which harbors a disk candidate around a B-type (proto)star.

Methods. We carried out ALMA Cycle 0 observations at $870 \mu \mathrm{m}$ of dust of typical high-density, molecular outflow, and cloud tracers with resolutions of $<0$ ' 5 . Complementary Subaru COMICS $25 \mu \mathrm{m}$ observations were carried out to trace the mid-infrared emission toward this star-forming region.

Results. The submillimeter continuum emission has revealed a filamentary structure fragmented into six cores, called A-F. The filament could be in quasi-equilibrium taking into account that the mass per unit length of the filament, 200-375 $M_{\odot} / \mathrm{pc}$, is similar to the critical mass of a thermally and turbulently supported infinite cylinder, $\sim 335 M_{\odot} / \mathrm{pc}$. The cores, which are on average separated by $\sim 0.02 \mathrm{pc}$, have deconvolved sizes of 1300-3400 AU, temperatures of 35-240 K, $\mathrm{H}_{2}$ densities $>10^{7} \mathrm{~cm}^{-3}$, and masses in the range 1-5 $M_{\odot}$, and they are subcritical. Core A, which is associated with a hypercompact HII region and could be the driving source of the molecular outflow observed in the region, is the most chemically rich source in G35.03+0.35 with strong emission of typical hot core tracers such as $\mathrm{CH}_{3} \mathrm{CN}$. Tracers of high density and excitation show a clear velocity gradient along the major axis of the core, which is consistent with a disk rotating about the axis of the associated outflow. The PV plots along the SE-NW direction of the velocity gradient show clear signatures of Keplerian rotation, although infall could also be present, and they are consistent with the pattern of an edge-on Keplerian disk rotating about a star with a mass in the range 5-13 $M_{\odot}$. The high $t_{\mathrm{ff}} / t_{\mathrm{rot}}$ ratio for core A suggests that the structure rotates fast and that the accreting material has time to settle into a centrifugally supported disk.

Conclusions. G35.03+0.35 is one of the most convincing examples of Keplerian disks rotating about high-mass (proto)stars. This supports theoretical scenarios according to which high-mass stars, at least B-type stars, would form through disk-mediated accretion.
\end{abstract}

Key words. ISM: individual objects: G35.03+0.35 - ISM: molecules - stars: formation - stars: kinematics and dynamics HII regions

\section{Introduction}

Understanding the formation of high-mass stars is one of the hot topics of current astrophysical research that have more

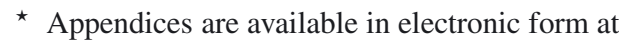
http://www. aanda.org quickly evolved in recent years. After a period where very different theoretical scenarios were proposed to explain the formation of OB-type stars, from non-spherical accretion (Nakano et al. 1995; Jijina \& Adams 1996) to stellar mergers (Bonnell et al. 1998), all theories appear to converge to a disk-mediated accretion scenario. In fact, either the monolithic collapse in turbulent core models (McKee \& Tan 2002; Krumholz et al. 2009), 


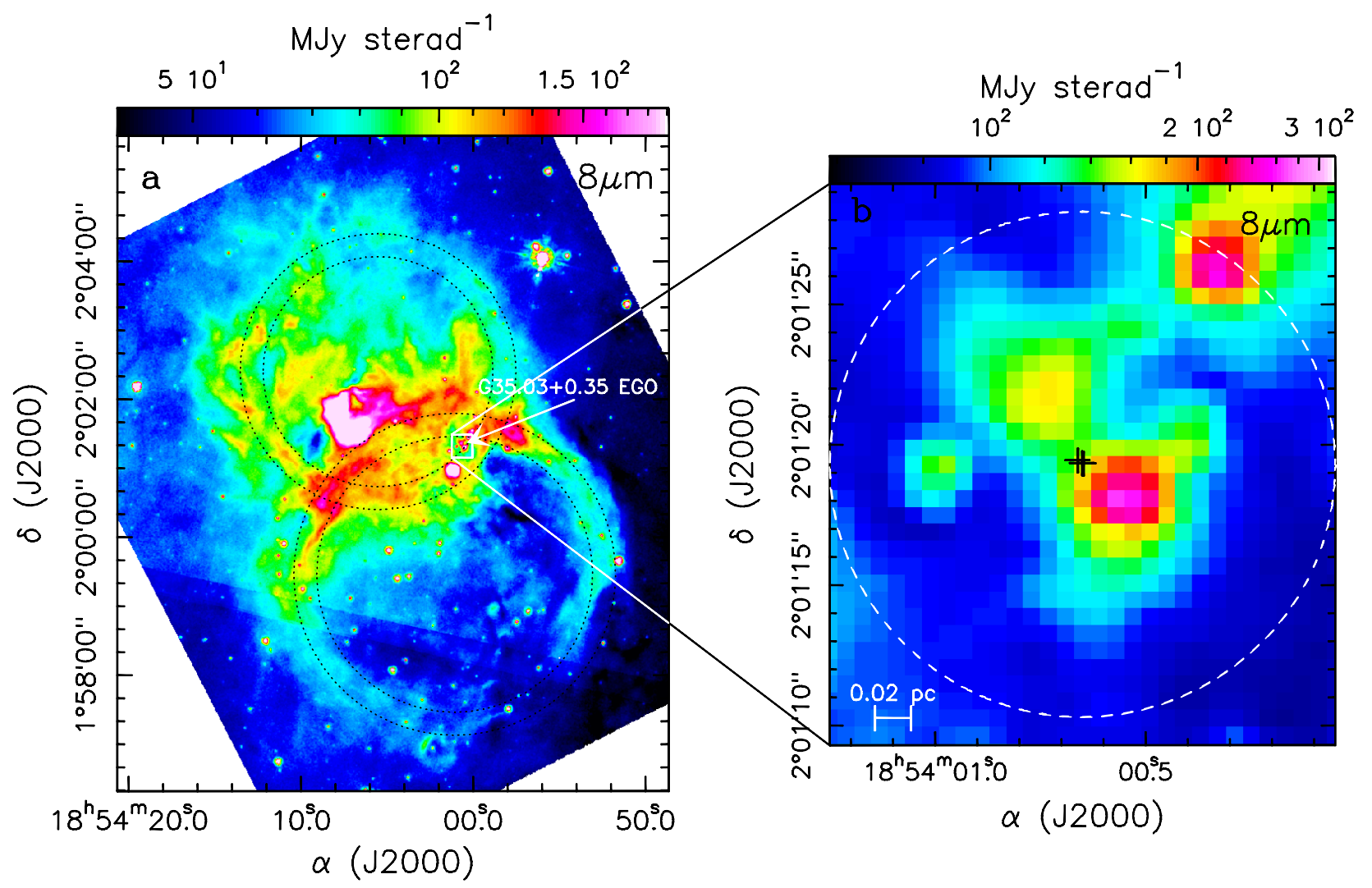

Fig. 1. a) Spitzer $8.0 \mu \mathrm{m}$ image on logarithmic scale toward N65 (Churchwell et al. 2006). The dotted circles outline the borders of the two bubbles. The white arrow indicates the G35.05+0.35 bipolar EGO identified by Cyganowski et al. (2008). b) Close-up toward the position of the G35.05+0.35 EGO showing the bipolar nebulosity at $8 \mu \mathrm{m}$. Black crosses indicate the positions of $\mathrm{H}_{2} \mathrm{O}$ masers (Forster \& Caswell 1999). The white circle denotes the primary beam of the ALMA observations.

the competitive accretion ones (Bonnell \& Bate 2006), or those proposing Bondi-Hoyle accretion (Keto 2007) predict the existence of a circumstellar accretion disk, in a sort of scaled-up version of low-mass star formation. However, while the existence of Keplerian rotating disks about low-mass young stellar objects (YSOs) and their importance have been assessed well (e.g., Burrows et al. 1996; Simon et al. 2000), the situation is less clear for higher mass protostars. In several cases, evidence of disks around high-mass YSOs have been reported in the literature (see Beltrán 2011 and references therein). However, only for very few B-type young stars has the disk kinematics been studied in detail and Keplerian rotation been proposed (e.g., Cesaroni et al. 2007; Wang et al. 2012). In fact, so far, no clear evidence of true accretion disks has yet been found around early O-type stars with masses $\gtrsim 20 M_{\odot}$ (Beltrán 2011), and only huge, massive, and transient rotating structures, called toroids, have been detected (see Beltrán et al. 2011a and references therein).

In a progressive effort to test the theoretical scenario of the formation of massive stars through disk-mediated accretion and to set the stage for a more challenging follow-up quest for disks around O-type stars, we carried out ALMA Cycle 0 observations of two IR disk candidates around B-type (proto)stars: G35.20-0.74N and G35.03+0.35. The first results for G35.20-0.74N have revealed velocity gradients consistent with an almost edge-on Keplerian disk (Sánchez-Monge et al. 2013).

In this work, we present the ALMA results for G35.03+0.35 (hereafter G35.03). This high-mass star-forming region is associated with the infrared (IR) source IRAS $18515+0157$ and has a local standard of rest (LSR) velocity, $V_{\mathrm{LSR}}$, of $\sim 49.5 \mathrm{~km} \mathrm{~s}^{-1}$, which corresponds to a kinematic distance of $\sim 3.2 \mathrm{kpc}$ using the rotation curve of Reid et al. (2009). This is the distance adopted in the rest of the paper. The source is associated with a bipolar nebulosity clearly visible at IR wavelengths (see Fig. 1b). Largescale IR observations have shown that G35.03 is located close to the border of the N65 bubble (Churchwell et al. 2006; see Fig. 1). Inspection of the Spitzer Galactic Legacy Infrared MidPlane Survey Extraordinaire (GLIMPSE; Benjamin et al. 2003; Churchwell et al. 2009) images reveals that the N65 bubble presents an eight-shaped morphology, which is more consistent with that of a bipolar nebula than a single bubble (see Fig. 1a). In this scenario, G35.03 would be located close to the waist of the double structure. Cyganowski et al. (2008) classified G35.03 as an extended green object (EGO) based on its $4.5 \mu \mathrm{m}$ emission (Fig. 2a). Interestingly, this EGO has a bipolar structure oriented northeast-southwest (NE-SW) that mirrors the eight-shaped morphology seen on the large scale (Fig. 1). The Spitzer MIPSGAL (Carey et al. 2009) $24 \mu \mathrm{m}$ image also reveals IR emission elongated in the direction of the bipolar nebula.

The G35.03 region has been studied in several molecular high-density tracers, such as $\mathrm{NH}_{3}$ (Harju et al. 1993; Codella et al. 2010; Brogan et al. 2011), CS (Anglada et al. 1996; Bronfman et al. 1996; Larionov et al. 1999), $\mathrm{H}^{13} \mathrm{CO}^{+}$, and $\mathrm{CH}_{3} \mathrm{OH}$ (Cyganowski et al. 2009). Interferometric centimeter continuum observations have revealed the presence of at least five compact sources (Cyganowski et al. 2011; see Fig. 2). One of them, labeled CM2 by Cyganowski et al. (2011), is located between the two $4.5 \mu \mathrm{m}$ lobes (Fig. 2a). This suggests that CM2 could be the driving source of the putative outflow traced by the extended, bipolar $4.5 \mu \mathrm{m}$ emission. The existence of a bipolar outflow associated with this EGO has been recently supported 


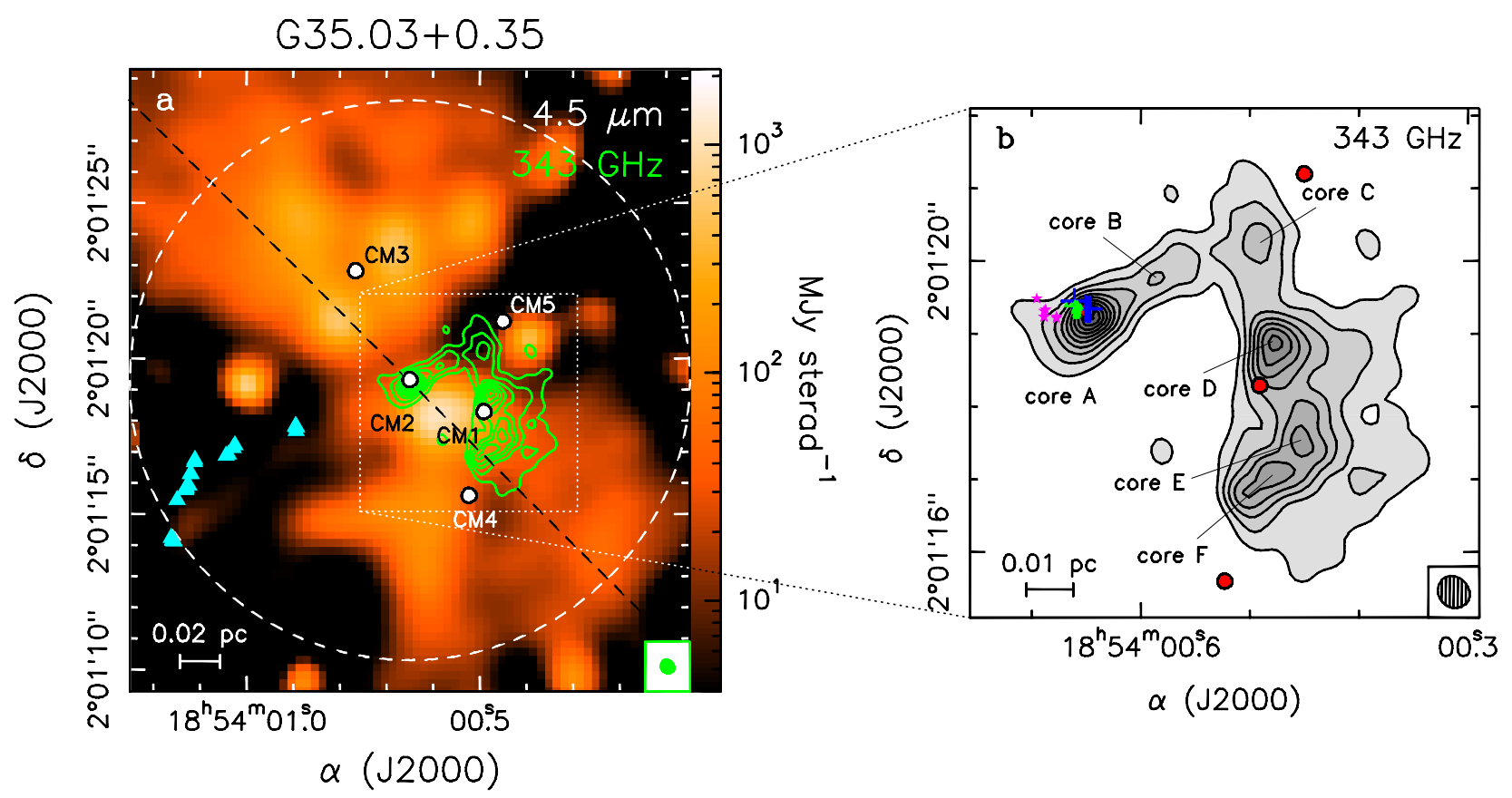

Fig. 2. a) Enhanced-resolution Spitzer $4.5 \mu \mathrm{m}$ image on logarithmic scale toward the star-forming region G35.03 overlaid with a contour map of the $343 \mathrm{GHz}$ continuum emission obtained with ALMA. The IR image has been obtained by applying the high resolution (HiRes) deconvolution algorithm to the Spitzer/IRAC data (Velusamy et al. 2008). The submillimeter map has been corrected for primary beam attenuation. The green contours range from 8 to $94.4 \mathrm{mJy}_{\text {beam }}{ }^{-1}$ in steps of $9.6 \mathrm{mJy}_{\text {beam }}{ }^{-1}$. The cyan triangles indicate the positions of $\mathrm{Class}^{4} 44 \mathrm{GHz} \mathrm{CH}_{3} \mathrm{OH} \mathrm{masers}$ (Cyganowski et al. 2009). The white circles indicate the positions of the 5 compact centimeter sources detected by Cyganowski et al. (2011). The dashed black line indicates the direction of the $4.5 \mu \mathrm{m}$ bipolar nebula. The dashed white circle denotes the primary beam of the ALMA $12 \mathrm{~m}$ antennas. The ALMA synthesized beam is shown in the lower right corner. b) Close-up of the central region that shows the $343 \mathrm{GHz}$ continuum emission map obtained with ALMA. Blue crosses and magenta stars indicate the positions of $\mathrm{H}_{2} \mathrm{O}$ and $\mathrm{OH}$ masers, respectively (Forster \& Caswell 1999), and green triangles those of Class II $6.7 \mathrm{GHz} \mathrm{CH}_{3} \mathrm{OH}$ masers (Cyganowski et al. (2009).

by the CO (3-2) observations of Paron et al. (2012; see their Fig. 10). Cyganowski et al. (2011) classify the centimeter source $\mathrm{CM} 2$ as a hypercompact (HC) HII region, with its $1.3 \mathrm{~cm}$ flux density being consistent with a single ionizing star of spectral type B1 based on the assumption of optically thin emission (Codella et al. 2010). This makes G35.03 one of the few EGOs associated with an HC HII region (Cyganowski et al. 2008). This centimeter source is associated with hot $\mathrm{NH}_{3}(6,6)$ emission (Brogan et al. 2011), $24 \mu \mathrm{m}$ IR emission, $\mathrm{H}_{2} \mathrm{O}$ and $\mathrm{OH}$ masers (Forster \& Caswell 1989, 1999; Baudry et al. 1997; Argon et al. 2000), and Class II 6.7 GHz CH${ }_{3} \mathrm{OH}$ masers (Menten 1991; Cyganowski et al. 2009; see Fig. 2b). Additionally, Cyganowski et al. (2009) also detected Class I $44 \mathrm{GHz} \mathrm{CH}_{3} \mathrm{OH}$ masers to the southeast of CM2 (Fig. 2a).

In Sect. 2 we describe the ALMA and Subaru observations; in Sect. 3 we present the results on the continuum, molecular line, and hot core emission toward G35.03, as well as the spectral energy distribution (SED) of this star-forming region; in Sect. 4 we analyze the temperature and mass estimates for the different cores in G35.03 and discuss the stability of the dust continuum structures, as well as the kinematics of the hot molecular core and of the large-scale line emission. Finally, in Sect. 5 we give our main conclusions.

\section{Observations}

\subsection{ALMA}

Interferometric observations of G35.03 were carried out with ALMA in Cycle 0 in May and June 2012 as part of the project 2011.0.00275.S (P.I.: R. Cesaroni). The source was observed at
Table 1. ALMA correlator setup.

\begin{tabular}{cc}
\hline \hline $\begin{array}{c}\text { Spectral } \\
\text { window }\end{array}$ & $\begin{array}{c}\text { Frequency range } \\
(\mathrm{MHz})\end{array}$ \\
\hline SPW0 & $336866.4-338741.4$ \\
SPW1 & $334982.5-336857.5$ \\
SPW2 & $348861.2-350736.2$ \\
SPW3 & $346908.6-348783.6$ \\
\hline
\end{tabular}

$870 \mu \mathrm{m}$ in Band 7 with the extended configuration with baselines in the range $36-400 \mathrm{~m}$, providing sensitivity to structures $\leq 2^{\prime \prime}$. The digital correlator was configured in four spectral windows (with dual polarization) of $1875 \mathrm{MHz}$ and 3840 channels each, which provide a spectral resolution of $\sim 0.4 \mathrm{~km} \mathrm{~s}^{-1}$. The spectral resolution of the line emission maps has been degraded to $0.6 \mathrm{~km} \mathrm{~s}^{-1}$. The frequency coverage of the four spectral windows of the correlator is given in Table 1 .

The phase reference center of the observations was set to the position $\alpha(\mathrm{J} 2000)=18^{\mathrm{h}} 54^{\mathrm{m}} 00^{\mathrm{s}} .65, \delta(\mathrm{J} 2000)=02^{\circ} 01^{\prime} 19^{\prime \prime} .30$. Flux, gain, and bandpass calibrations were achieved through observations of Neptune and J1751+096. The data were calibrated and imaged using the $\mathrm{CASA}^{1}$ software package. Further imaging and analysis was done with the GILDAS ${ }^{2}$ software package. The continuum map was created from the line-free channels. We subtracted the continuum from the line emission directly in the $(u, v)$-domain. Continuum and channel maps were created

\footnotetext{
1 The CASA package is available at http://casa.nrao.edu/ 2 The GILDAS package is available at http://www.iram.fr/ IRAMFR/GILDAS
} 
Table 2. Parameters of the maps ${ }^{\mathrm{a}}$.

\begin{tabular}{|c|c|c|c|c|}
\hline \multirow[b]{2}{*}{ Observation } & \multirow[b]{2}{*}{$\begin{array}{c}\text { Frequency } \\
\quad(\mathrm{GHz})\end{array}$} & \multirow[b]{2}{*}{$\begin{array}{c}E_{\text {upper }} \\
(\mathrm{K})\end{array}$} & \multicolumn{2}{|c|}{ Synthesized beam } \\
\hline & & & $\begin{array}{l}H P B W \\
(\operatorname{arcsec})\end{array}$ & $\begin{array}{c}\text { PA } \\
(\mathrm{deg})\end{array}$ \\
\hline continuum & 343.000 & & $0.47 \times 0.41$ & 45 \\
\hline $\mathrm{CH}_{3} \mathrm{OH}\left(2_{2,1}-3_{1,2}\right)$ & 335.133 & 45 & $0.49 \times 0.44$ & 38 \\
\hline $\mathrm{SO}_{2}\left(23_{3,21}-23_{2,22}\right)$ & 336.089 & 276 & $0.50 \times 0.43$ & 37 \\
\hline $\mathrm{CH}_{3} \mathrm{OH}\left(14_{7,7}-15_{6,9}\right)$ & 336.438 & 488 & $0.50 \times 0.43$ & 38 \\
\hline $\mathrm{HC}_{3} \mathrm{~N}(37-36)$ & 336.520 & 307 & $0.50 \times 0.43$ & 38 \\
\hline $\operatorname{SO}\left(10_{11}-10_{10}\right)$ & 336.553 & 143 & $0.50 \times 0.43$ & 38 \\
\hline $\mathrm{C}^{17} \mathrm{O}(3-2)$ & 337.061 & 32 & $0.49 \times 0.43$ & 42 \\
\hline $\mathrm{CH}_{3} \mathrm{OH}\left(7_{1,7}-6_{1,6}\right) v_{t}=1$ & 337.297 & 390 & $0.49 \times 0.43$ & 40 \\
\hline$C^{34} S(7-6)$ & 337.396 & 50 & $0.49 \times 0.43$ & 40 \\
\hline $\mathrm{CH}_{3} \mathrm{OH}\left(7_{2,6}-6_{2,5}\right)$ & 338.513 & 103 & $0.49 \times 0.42$ & 39 \\
\hline $\mathrm{H}^{13} \mathrm{CO}^{+}(4-3)$ & 346.998 & 42 & $0.48 \times 0.42$ & 37 \\
\hline $\mathrm{SiO}(8-7)$ & 347.330 & 75 & $0.48 \times 0.42$ & 36 \\
\hline $\mathrm{H}_{2} \mathrm{CS}\left(10_{1,9}-9_{1,8}\right)$ & 348.531 & 105 & $0.48 \times 0.41$ & 35 \\
\hline $\mathrm{CH}_{3}^{13} \mathrm{CN}\left(19_{K}-18_{K}\right)$ & $349.280^{\mathrm{b}}$ & $168-282$ & $0.47 \times 0.41$ & 36 \\
\hline $\mathrm{C}_{2} \mathrm{H}\left(4_{9 / 2,5}-3_{7 / 2,4}\right)$ & 349.337 & 42 & $0.48 \times 0.41$ & 35 \\
\hline $\mathrm{CH}_{3} \mathrm{CN}\left(19_{K}-18_{K}\right)$ & $349.453^{b}$ & $168-1209$ & $0.47 \times 0.41$ & 36 \\
\hline $\operatorname{HNCO}\left(16_{1,16}-15_{1,15}\right)$ & 350.333 & 186 & $0.48 \times 0.41$ & 36 \\
\hline $\mathrm{CH}_{3} \mathrm{CN}(K, l) v_{8}=1$ & $350.552^{\mathrm{c}}$ & $648-1709$ & $0.48 \times 0.41$ & 36 \\
\hline
\end{tabular}

Notes. ${ }^{(a)}$ Only for those molecular lines analyzed in this paper. ${ }^{(b)}$ The frequency is that of the $K=0$ component. ${ }^{(c)}$ The frequency is that of the $K, l=2,+1$ component.

with the ROBUST parameter of Briggs (1995) set equal to 0.5. Details of the rest frequency and synthesized CLEANed beam of the maps are given in Table 2. The rms noise of the maps is $3 \mathrm{mJy} \mathrm{beam}^{-1}$ for the continuum and $\sim 15 \mathrm{mJy} \mathrm{beam}^{-1}$ per channel of $0.6 \mathrm{~km} \mathrm{~s}^{-1}$ for the line maps.

\subsection{Subaru}

We carried out mid-infrared imaging observations at $25 \mu \mathrm{m}$ toward G35.03 on 2011 August 25 in Hawaii Standard Time using the COMICS camera (Kataza et al. 2000) at the Cassegrain focus of the $8.2 \mathrm{~m}$ Subaru Telescope. We configured the Q24.5 filter and employed chopping-and-nodding mode for subtracting the sky-background emission and their time variations. The camera provides a field of view of $\sim 42^{\prime \prime} \times 32^{\prime \prime}$, with a pixel size of 0 '.13. Flux calibration for the night was performed toward two standard sources of HD146051 and HD161096 listed in Cohen et al. (1999). We estimated the overall uncertainty in the flux calibration to be less than $10 \%$.

The astrometric calibration of the image was obtained by the alignment to the corresponding GLIMPSE/IRAC $8 \mu \mathrm{m}$ image, after smoothing the former to the angular resolution of the latter $\left(\sim 2^{\prime \prime}\right)$. The match between the two images was obtained by visual inspection, which results in a relative astrometric accuracy of $\sim 0$.'4. Taking into account that the positional uncertainty of the GLIMPSE survey is $\$ 0$ '. 2 , we infer an absolute astrometric error of the Subaru image of $\sim 0$.' $^{\prime}$.

\section{Results}

\subsection{Continuum emission}

Figure 3 shows the $870 \mu \mathrm{m}$ continuum emission map from ALMA overlaid on the $250 \mu \mathrm{m}$ Herschel Infrared Galactic Plane

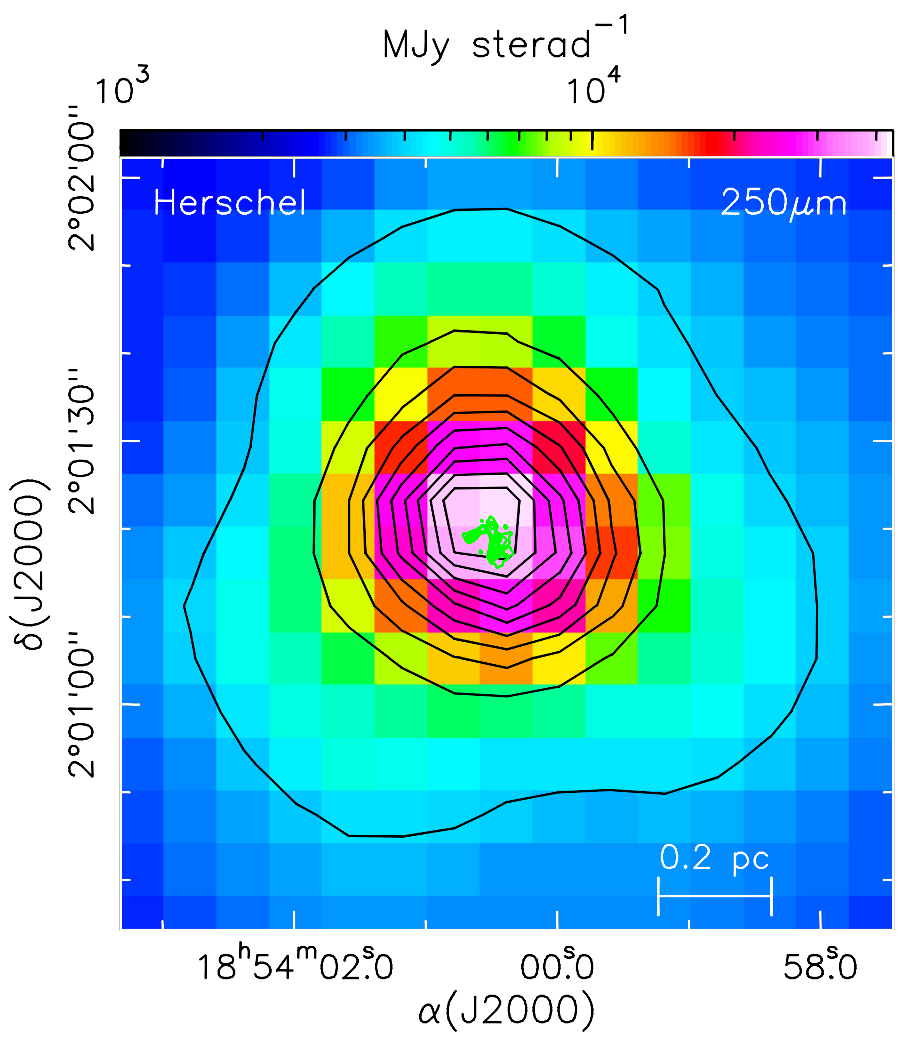

Fig. 3. Overlay of the ALMA $343 \mathrm{GHz}$ continuum emission (green contours) map on the Herschel $250 \mu \mathrm{m}$ map from the Hi-GAL survey (image and black contours). Green contours are the same as in Fig. 2. Black contours are 4000 to $40000 \mathrm{MJy} s t e r a d^{-1}$ in steps of $4000 \mathrm{MJy} \mathrm{sterad}^{-1}$.

Survey (Hi-GAL; Molinari et al. 2010a,b) map of the G35.03 core. The Hi-GAL map, with an angular resolution of $\sim 18^{\prime \prime}$, reveals a single roundish clump with a size (at a $3 \sigma$ level) of $\sim 67^{\prime \prime}$ $(\sim 1 \mathrm{pc})$. On the other hand, the high angular resolution $\left(\sim 00^{\prime} \cdot 44\right)$ ALMA observations reveal a complete different structure of the core on small scales, as clearly seen in Fig. 2. The spherically symmetric morphology observed at parsec scales disappears and the small scale emission unveils a filamentary U-shaped structure with one branch of the "U" lying across the waist of the NE-SW bipolar nebula traced by the $4.5 \mu$ m emission (Fig. $2 \mathrm{a}$ ). This structure appears to wrap around the brightest $4.5 \mu \mathrm{m}$ peak, located in the southern lobe of the bipolar nebula. The nature of this IR peak will be discussed in Sect. 4.4.

This filamentary dust structure fragments into six cores that we have identified by visual inspection and called A-F (Fig. 2b). The cores are on average separated by $1^{\prime \prime}$, i.e., $\sim 0.02$ pc. Cores A and D appear to be associated with the compact centimeter sources CM2 and CM1, respectively, which, according to Cyganowski et al. (2011), would be HC or ultracompact (UC) HII regions. Core A, which is the strongest at submillimeter wavelengths (Table 3), lies at the center of the bipolar nebula. Since extended $4.5 \mu \mathrm{m}$ emission is thought to trace shocked molecular gas in protostellar outflows (Cyganowski et al. 2008), core A could be the driving source of such an outflow because it is located at the center of symmetry of the bipolar nebula. Paron et al. (2012) have detected outflowing activity toward the bipolar nebula, but the angular resolution of their CO (3-2) observations $\left(\sim 22^{\prime \prime}\right)$ is insufficient for discerning the driving source. The position, fluxes, and deconvolved diameters of the cores are given in Table 3 . The deconvolved sizes are the average diameters of the $50 \%$ contour level calculated assuming that the cores 
Table 3. Positions, flux densities, and diameters of the $\operatorname{cores}^{a}$.

\begin{tabular}{|c|c|c|c|c|c|c|}
\hline \multirow[b]{2}{*}{ Core } & \multicolumn{2}{|c|}{ Position peak } & \multirow[b]{2}{*}{$\begin{array}{c}I_{v}^{\text {peak }, b} \\
(\mathrm{mJy} / \text { beam })\end{array}$} & \multirow[b]{2}{*}{$\begin{array}{c}S_{v}^{b} \\
(\mathrm{mJy})\end{array}$} & \multicolumn{2}{|c|}{ Source diameter ${ }^{c}$} \\
\hline & $\begin{array}{c}\alpha(\mathrm{J} 2000) \\
\mathrm{h} \mathrm{m} \mathrm{s}\end{array}$ & $\begin{array}{c}\delta(\mathrm{J} 2000) \\
\circ, \prime \prime\end{array}$ & & & $\begin{array}{c}\theta_{\mathrm{s}} \\
(\operatorname{arcsec})\end{array}$ & $\begin{array}{c}D_{\mathrm{s}} \\
(\mathrm{AU})\end{array}$ \\
\hline $\mathrm{A}^{d}$ & 18540.645 & 020119.23 & $96 \pm 3$ & $225 \pm 9$ & $0.41 \pm 0.05$ & $1310 \pm 160$ \\
\hline B & 18540.586 & 020119.78 & $29 \pm 3$ & $70 \pm 2$ & $0.70 \pm 0.13$ & $2240 \pm 420$ \\
\hline $\mathrm{C}$ & 18540.490 & 020120.26 & $32 \pm 3$ & $147 \pm 3$ & $1.05 \pm 0.20$ & $3360 \pm 640$ \\
\hline $\mathrm{D}^{e}$ & 18540.479 & 020118.90 & $69 \pm 3$ & $340 \pm 9$ & $0.81 \pm 0.09$ & $2600 \pm 290$ \\
\hline $\mathrm{E}$ & 18540.453 & 020117.54 & $51 \pm 3$ & $254 \pm 7$ & $0.85 \pm 0.09$ & $2720 \pm 290$ \\
\hline $\mathrm{F}$ & 18540.479 & 020117.06 & $54 \pm 3$ & $226 \pm 7$ & $0.75 \pm 0.07$ & $2400 \pm 220$ \\
\hline
\end{tabular}

Notes. ${ }^{(a)}$ Derived from the continuum dust emission. ${ }^{(b)}$ Peak intensity and integrated flux density corrected for primary beam response. (c) Deconvolved average diameter, calculated assuming that the cores are Gaussians from the expression $\theta_{\mathrm{s}}=\sqrt{F W H M^{2}-H P B W^{2}}$, where $F W H M=2 \sqrt{A / \pi}$, with $A$ the area inside the polygon corresponding to the contour at half maximum, and HPBW is the synthesized beam. (d) Associated with the centimeter continuum source CM2, $\alpha(\mathrm{J} 2000)=18^{\mathrm{h}} 54^{\mathrm{m}} 00{ }^{\mathrm{s}} 6498, \delta(\mathrm{J} 2000)=02^{\circ} 01^{\prime} 199^{\prime} 32$ (Cyganowski et al. 2011). ${ }^{(e)}$ Associated with the centimeter continuum source CM1, $\alpha(\mathrm{J} 2000)=18^{\mathrm{h}} 54^{\mathrm{m}} 00^{\lessgtr} 49098, \delta(\mathrm{J} 2000)=02^{\circ} 01^{\prime} 18^{\prime \prime} 292$ (Cyganowski et al. 2011).

are Gaussians. The cores have sizes ranging from $\sim 1300 \mathrm{AU}$ for core A to $\sim 3400$ AU for core $\mathrm{C}$.

The dusty filament has an integrated flux density of $1.3 \mathrm{Jy}$. The peak flux of the $870 \mu \mathrm{m}$ APEX Telescope Large Survey of the Galaxy (ATLASGAL; Schuller et al. 2009) map of G35.03, which has an angular resolution of $19 ! 2$, is $5.8 \mathrm{Jy} \mathrm{beam}^{-1}$. Therefore, by comparing the ALMA and ATLASGAL values, we estimated that the continuum ALMA Cycle 0 observations are only recovering $\sim 20 \%$ of the total flux of the compact core.

\subsection{Molecular line emission}

Figure 4 shows the spectra of the lower and upper ALMA sidebands around 337 and $349 \mathrm{GHz}$, respectively, toward the six cores detected in the continuum. The spectra were obtained toward the continuum peak position of each core. Clearly, core A is the most chemically rich, since the corresponding spectrum displays a forest of molecular lines, which include several transitions of $\mathrm{CH}_{3} \mathrm{OH}, \mathrm{H}^{13} \mathrm{CO}^{+}, \mathrm{C}_{2} \mathrm{H}, \mathrm{C}^{17} \mathrm{O}, \mathrm{H}_{2} \mathrm{CS}, \mathrm{HC}_{3} \mathrm{~N}, \mathrm{SiO}$, $\mathrm{C}^{34} \mathrm{~S}, \mathrm{SO}, \mathrm{SO}_{2}$, and typical hot core tracers, such as $\mathrm{CH}_{3} \mathrm{CN}$ and HNCO. A detailed analysis of the molecular line emission toward this core will be the subject of another study (Allen et al., in prep.). In the present work, we focus only on a few of these hot core tracers, those with the strongest line emission and that are blended the least with other species, namely $\mathrm{CH}_{3} \mathrm{CN}$, $\mathrm{CH}_{3}^{13} \mathrm{CN}$, vibrationally excited $\mathrm{CH}_{3} \mathrm{CN}, \mathrm{CH}_{3} \mathrm{OH}$, torsionally excited $\mathrm{CH}_{3} \mathrm{OH}, \mathrm{HC}_{3} \mathrm{~N}, \mathrm{HNCO}, \mathrm{SO}$, and $\mathrm{SO}_{2}$. These species allow us to cover a broad range of excitation energies from $\sim 30 \mathrm{~K}$ to $\sim 1700 \mathrm{~K}$ (see Table 2).

The only other core that shows $\mathrm{CH}_{3} \mathrm{CN}$ emission, although weak, is core $\mathrm{D}$, which is associated with the UC HII region $\mathrm{CM} 1$. The rest of the cores present emission of more abundant species, such as $\mathrm{CH}_{3} \mathrm{OH}, \mathrm{H}^{13} \mathrm{CO}^{+}, \mathrm{C}_{2} \mathrm{H}, \mathrm{C}^{17} \mathrm{O}, \mathrm{H}_{2} \mathrm{CS}, \mathrm{HC}_{3} \mathrm{~N}$, and $\mathrm{C}^{34} \mathrm{~S}$. As will be discussed in the next section, the emission of all these molecules appears to be extended. That cores B, C, $\mathrm{E}$, and $\mathrm{F}$ do not show emission of hot core tracers suggests that, unlike cores A and D, they either contain lower mass YSOs or are still in a very early evolutionary phase prior to the development of a young massive star.

\subsubsection{Large-scale molecular line emission}

Figure 5 shows the $343 \mathrm{GHz}$ continuum emission overlaid as contours over the integrated intensity (moment 0 ), line velocity (moment 1), and velocity dispersion (moment 2) maps of $\mathrm{H}^{13} \mathrm{CO}^{+}, \mathrm{C}_{2} \mathrm{H}, \mathrm{C}^{17} \mathrm{O}, \mathrm{H}_{2} \mathrm{CS}$, and $\mathrm{C}^{34} \mathrm{~S}$. The emission of these species is extended and well correlated with the dust continuum emission, at least toward the western and southern regions. In fact, the line emission toward W-SW nicely trace the arc-like filament seen in the continuum. The gas emission seems to surround the $4.5 \mu \mathrm{m}$ emission peak (Fig. 2), outlining a cavity. This cavity is perfectly encircled by the gas emission, whereas the dust emission is not detected to the E-SE. The line velocity maps in Fig. 5 show a clear velocity gradient, with the velocity increasing from NE to SW. On the other hand, the velocity dispersion maps show an almost constant line width of $\sim 2 \mathrm{~km} \mathrm{~s}^{-1}$ throughout the region, except for a remarkable maximum of $\sim 10 \mathrm{~km} \mathrm{~s}^{-1}$ southward of core A, exactly where the dust emission disappears. This enhancement of the velocity dispersion is best seen in $\mathrm{C}^{34} \mathrm{~S}$. In Sect. 4.4, we discuss a possible origin of these emission features.

The other species that shows extended emission is $\mathrm{SiO}$. Figure 6 shows the $343 \mathrm{GHz}$ continuum emission overlaid as contours over the integrated intensity (moment 0 ) $\mathrm{SiO}$ map. This figure also shows the $\mathrm{SiO}$ spectra toward selected positions in the G35.03 core. The $\mathrm{SiO}$ emission is clearly associated with core A, but it is also detected in other parts of the G35.03 core, although unlike the other extended species, it is less correlated with the dust continuum emission. In fact, it is only clearly visible toward the N and NW of core A and, more marginally, toward the position of cores D and E. As for all the species that show extended emission and that have been observed in these Cycle 0 observations, the $\mathrm{SiO}$ maps are strongly affected by sidelobes and missing flux. However, in the case of $\mathrm{SiO}$, the filtering of emission is so important that it prevents us from properly interpreting the extended emission. In any case, we think that the extended $\mathrm{SiO}$ emission is associated with that of $\mathrm{H}^{13} \mathrm{CO}^{+}, \mathrm{C}_{2} \mathrm{H}$, $\mathrm{C}^{17} \mathrm{O}, \mathrm{H}_{2} \mathrm{CS}$, and $\mathrm{C}^{34} \mathrm{~S}$, and this will be discussed with that of these other species in Sect. 4.4.

\subsubsection{Hot core emission toward core $A$}

Figure 7 shows a zoom of the spectrum toward core A where the different $K$-components of the typical hot core tracer methyl cyanide and its isotopologue are visible. As seen in the top panel, all components of $\mathrm{CH}_{3} \mathrm{CN}$ up to $K=9$ (with an upper level energy of $745 \mathrm{~K}$ ) have been detected. Regarding $\mathrm{CH}_{3}^{13} \mathrm{CN}$, all the lines up to the $K=4$ component have been clearly 

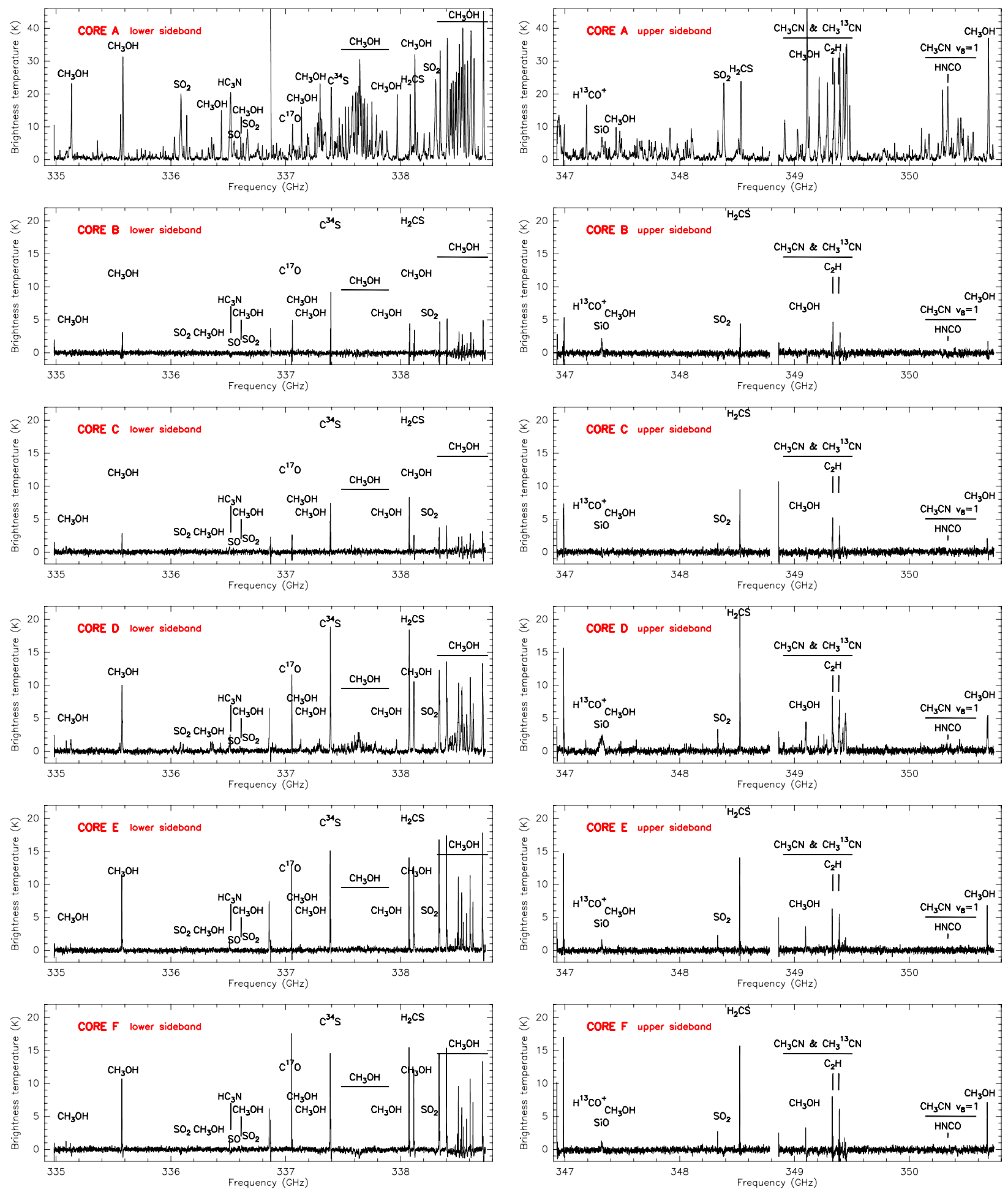

Fig. 4. Spectra toward the continuum peak position of the six cores identified in the continuum image at $343 \mathrm{GHz}$ (see Fig. 2). The spectra shown cover the whole frequency range of the ALMA observations: $334.982-338.741 \mathrm{GHz}$ in the lower sideband, and 346.908-350.736 GHz in the upper sideband. The brightness temperature scale of core A (the richest and brightest core) is different from that of the rest of the cores. The absorption visible in some spectra is probably produced by the interferometer filtering out part of the extended emission.

detected. Because of the blending with a $\mathrm{CH}_{3} \mathrm{OH}$ transition, it is not possible to confirm the detection of the $K=5$ component. Furthermore, a number of $\mathrm{CH}_{3} \mathrm{CN} v_{8}=1$ transitions, with upper level energies ranging from $\sim 690$ to $\sim 1040 \mathrm{~K}$, have been detected.
Figures 8,9, and 10 show the integrated intensity (moment 0 ) maps toward core $\mathrm{A}$ of the $\mathrm{CH}_{3} \mathrm{CN} K=2$ and $8, \mathrm{CH}_{3}^{13} \mathrm{CN}$ $K=2$, and $\mathrm{CH}_{3} \mathrm{CN} v_{8}=1(K, l)=(3,1)$ lines, various transitions of $\mathrm{CH}_{3} \mathrm{OH}$ and $\mathrm{CH}_{3} \mathrm{OH} v_{\mathrm{t}}=1$, with upper level energies from 45 to $390 \mathrm{~K}$, and $\mathrm{SO}, \mathrm{HNCO}, \mathrm{SO}_{2}$, and $\mathrm{HC}_{3} \mathrm{~N}$ with upper 


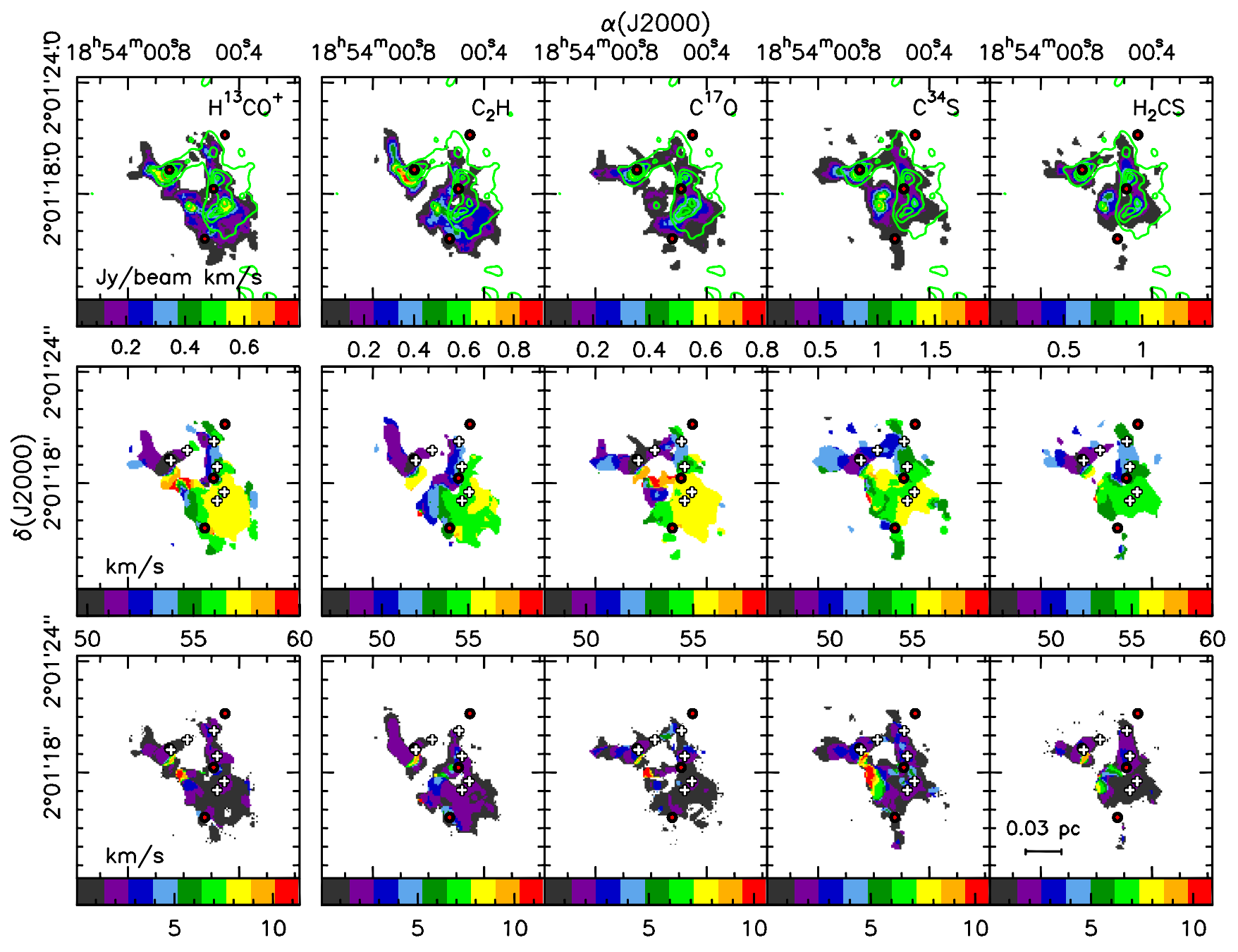

Fig. 5. Top panels: overlay of the $343 \mathrm{GHz}$ continuum emission (green contours) on the integrated intensity (moment 0$)$ map $\left(\right.$ colors) of $\mathrm{H}^{13} \mathrm{CO}^{+}$, $\mathrm{C}_{2} \mathrm{H}, \mathrm{C}^{17} \mathrm{O}, \mathrm{C}^{34} \mathrm{~S}$, and $\mathrm{H}_{2} \mathrm{CS}$. The green contours range from 8 to $84.8 \mathrm{mJy}_{\text {beam }}^{-1}$ in steps of $19.2 \mathrm{mJy}^{-1}$ beam ${ }^{-1}$ Middle panels: line velocity (moment 1) maps for the same molecular species. Bottom panels: velocity dispersion (moment 2) maps for the same species. The red circles indicate the positions of the 5 compact centimeter sources detected by Cyganowski et al. (2011), and the white crosses the positions of the submillimeter continuum cores (Table 3 ).

level energies from 143 to $307 \mathrm{~K}$. In all these maps, the emission appears compact and concentrated toward the position of the dust emission peak. In fact, gas and dust roughly peak at the same position, which is also roughly coincident with that of the centimeter source CM2 detected by Cyganowski et al. (2011). The emission is resolved in all the species and elongated mostly in the SE-NW direction. The PA of the major axis of the hot molecular core (HMC) was estimated, after Gaussian deconvolution, with 2D-Gaussian fits to the integrated intensity maps of the most resolved species and ranges from $\sim-25^{\circ}$ to $\sim-35^{\circ}$. Therefore, the PA is roughly perpendicular to the axis of the $4.5 \mu \mathrm{m}$ bipolar nebula. The integrated emission of the $\mathrm{CH}_{3}^{13} \mathrm{CN}$ $K=2$ transition (Fig. 8c) shows a faint southern tail likely not associated with $\mathrm{CH}_{3}^{13} \mathrm{CN} K=2$ but with the emission of other species blended with it. Possible blended candidates could be vinyl cyanide, trans-ethanol, and/or ethyl cyanide.

\subsection{Spectral energy distribution}

The SED of the G35.03 star-forming region (see Fig. 11) has been obtained by integrating over a region of $\sim 2^{\prime}$ the images of Hi-GAL (Molinari et al. 2010a). These were complemented by ancillary data from GLIMPSE (Benjamin et al. 2003), COMICS at the Subaru Telescope, the Midcourse Space Experiment (MSX; Price et al. 1999), Spitzer/MIPSGAL (Carey et al. 2009), Wide-field Infrared Survey Explorer (WISE; Wright et al. 2010), ATLASGAL (Schuller et al. 2009), and the CSO Bolocam Galactic Plane Survey (BGPS; Drosback et al. 2008). Figures A.1 and A.2 show the IR and submillimeter images of G35.03 at representative wavelengths. The bolometric luminosity, $L_{\text {bol }}$, was computed by integrating the SED over the whole range for which measurements are available, and it turns out to be $\sim 2 \times 10^{4} L_{\odot}$. This value is greater than the stellar luminosity of $6.3 \times 10^{3} L_{\odot}$ estimated for the centimeter continuum source CM2 that is associated with core A. This stellar luminosity estimate has been obtained from the $1.3 \mathrm{~cm}$ emission, assuming that it is optically thin (Codella et al. 2010) and following the tables of Davies et al. (2011) and Mottram et al. (2011), which tabulate the ZAMS stellar parameters (temperature, luminosity, and radius) and the spectral type as a function of mass and Lyman photon flux. These authors used the calculations of Martins et al. (2005) and Lanz \& Hubeny (2007) for the Lyman photon fluxes as a function of temperature for $\mathrm{O}$ and $\mathrm{B}$ stars. 

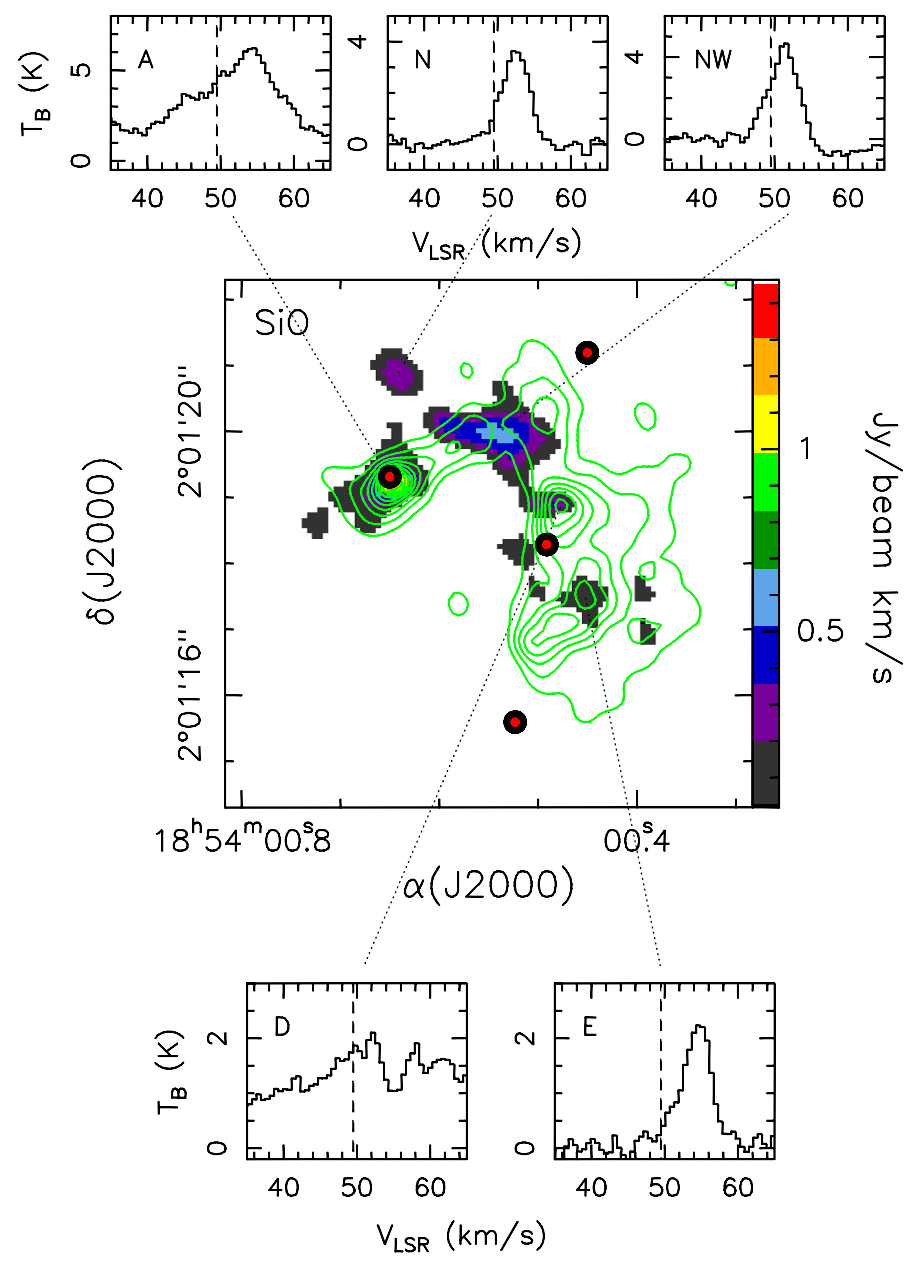

Fig. 6. Middle panel: overlay of the $343 \mathrm{GHz}$ continuum emission (green contours) on the integrated intensity (moment 0 ) map (colors) of $\mathrm{SiO}(8-7)$. The contour levels are the same as in Fig. 2. The red circles indicate the positions of the 5 compact centimeter sources detected by Cyganowski et al. (2011). Lower and upper panels: $\mathrm{SiO}(8-7)$ spectra toward selected positions in the G35.03 core. The vertical dashed line indicates the $V_{\mathrm{LSR}}$.

However, the SED was computed by integrating the emission over a region of $\sim 2^{\prime}$, which also includes the emission of the UC HII region CM1. According to Codella et al. (2010), the $1.3 \mathrm{~cm}$ flux density of CM1 would be consistent with a single ionizing star of spectral type B0.5, corresponding to a stellar luminosity of $1.1 \times 10^{4} L_{\odot}$ (Mottram et al. 2011). Therefore, the stellar luminosity of the whole star-forming region would be $\sim 1.7 \times 10^{4} L_{\odot}$, which is consistent with the value obtained by integrating the SED.

\section{Analysis and discussion}

\subsection{Temperature and mass estimates}

Methyl cyanide has only been clearly detected toward core A and marginally toward core D. Table 4 shows the $\mathrm{CH}_{3} \mathrm{CN}$, $\mathrm{CH}_{3}{ }^{13} \mathrm{CN}$, and $\mathrm{CH}_{3} \mathrm{CN} v_{8}=1(19-18)$ line parameters derived by fitting with Gaussian profiles the spectra toward core A obtained by averaging the emission inside an area corresponding to the $3 \sigma$ contour level of $\mathrm{CH}_{3} \mathrm{CN} K=2$. On the other hand, a large number of methanol transitions have been detected toward all the cores in G35.03. Because of this, we used $\mathrm{CH}_{3} \mathrm{OH}$ to estimate the excitation temperature $T_{\text {ex }}$. Taking
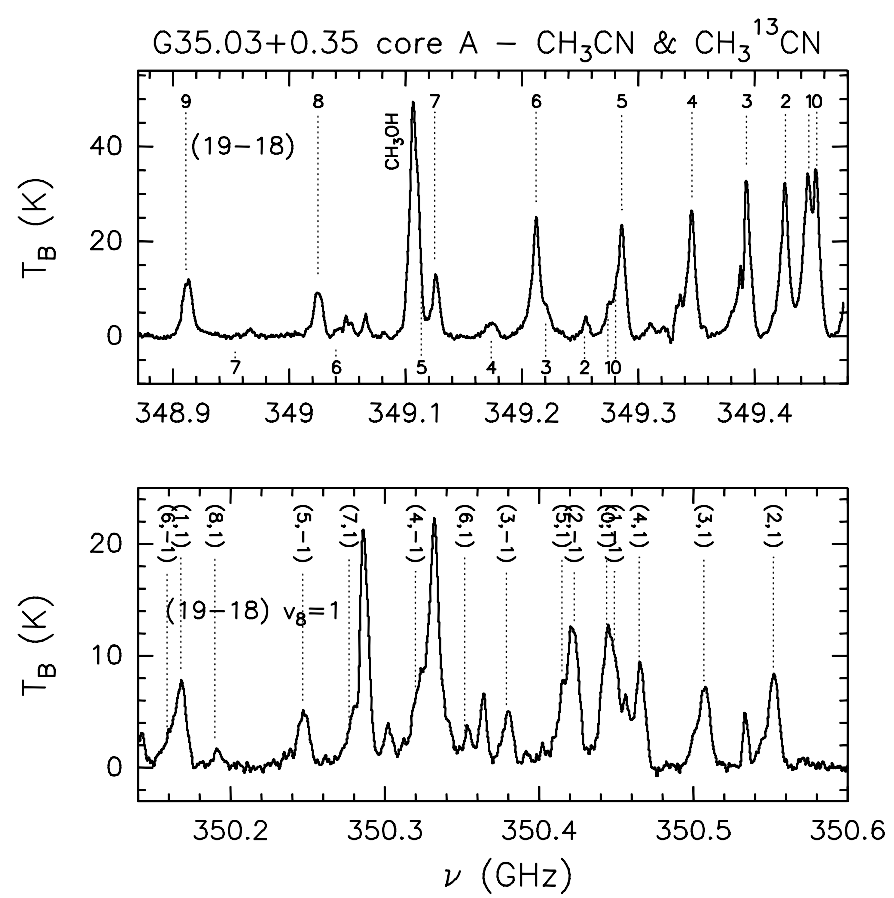

Fig. 7. Top panel: $\mathrm{CH}_{3} \mathrm{CN}$ and $\mathrm{CH}_{3}^{13} \mathrm{CN}$ (19-18) spectra obtained toward the continuum peak position of core $\mathrm{A}$. The different $K$-components of $\mathrm{CH}_{3} \mathrm{CN}$ and $\mathrm{CH}_{3}^{13} \mathrm{CN}$ (given in the upper and lower part of the panel, respectively) are marked with dashed lines. Bottom panel: $\mathrm{CH}_{3} \mathrm{CN}(19-18)$ vibrationally excited $\left(v_{8}=1\right)$ spectra for which the different $K, l$-components are also marked with dashed lines.

the high gas densities $\left(>10^{7} \mathrm{~cm}^{-3}\right)$ estimated toward the cores (Table 4) into account, the methanol $T_{\mathrm{ex}}$ should be similar to the kinetic temperature. The methanol ground-state and torsionally excited transitions were fitted using the CASA interface of the XCLASS software called myXCLASS described in Schilke et al. (2001), Comito et al. (2005), and Zernickel et al. (2012). The myXCLASS software makes use of the CDMS (Cologne Database for Molecular Spectroscopy; Müller et al. 2001) and JPL (Jet Propulsion Laboratory; Pickett et al. 1998) spectroscopic databases to simulate a synthetic spectrum of a particular species, depending on the five input parameters provided. These parameters are the abundance of the molecule, excitation temperature, size of the source, velocity, and line width. The program assumes that these quantities are the same for all transitions. Furthermore, myXCLASS takes line opacity into account. The best fit of the observed spectrum is obtained by minimizing the $\chi^{2}$ of the five input parameters with the program MAGIX (Modeling and Analysis Generic Interface for eXternal numerical codes; Bernst et al. 2011; Möller et al. 2013). Therefore, the combination of myXCLASS and MAGIX provides the best fits, hence the column density and $T_{\text {ex }}$ of the observed species, which in our case is $\mathrm{CH}_{3} \mathrm{OH}$. Figures B.1 to B.6 show the observed and fitted $\mathrm{CH}_{3} \mathrm{OH}$ lines toward all the cores in G35.03. The fit includes ground-state $\mathrm{CH}_{3} \mathrm{OH}$ and ${ }^{13} \mathrm{CH}_{3} \mathrm{OH}$ and torsionally excited $\mathrm{CH}_{3} \mathrm{OH}$ lines. We assumed a relative abundance $\left[{ }^{12} \mathrm{C} /{ }^{13} \mathrm{C}\right]$ of 54, estimated following Wilson \& Rood (1994) for a galactocentric distance of $6.2 \mathrm{kpc}$.

Table 5 shows $T_{\text {ex }}$ and $N_{\mathrm{CH}_{3} \mathrm{OH}}$ for each core. As seen in this table, cores $\mathrm{A}$ and $\mathrm{D}$, which are those associated with $\mathrm{HC}$ and/or UC HII regions, are the hottest with $T_{\mathrm{ex}}>100 \mathrm{~K}$, and those with the highest methanol column densities as well, $N_{\mathrm{CH}_{3} \mathrm{OH}} \sim 5 \times$ $10^{17}-3.2 \times 10^{19} \mathrm{~cm}^{-2}$. The rest of the cores have temperatures 
M. T. Beltrán et al.: The high-mass star-forming region G35.03+0.35 imaged with ALMA

Table 4. $\mathrm{CH}_{3} \mathrm{CN}, \mathrm{CH}_{3}{ }^{13} \mathrm{CN}$, and $\mathrm{CH}_{3} \mathrm{CN}$ (19-18) $v_{8}=1$ line parameters toward core $\mathrm{A}$.

\begin{tabular}{|c|c|c|c|c|}
\hline $\begin{array}{l}\text { Line } \\
K \\
\end{array}$ & $\begin{array}{c}V_{\mathrm{LSR}} \\
\left(\mathrm{km} \mathrm{s}^{-1}\right) \\
\end{array}$ & $\begin{array}{c}F W H M \\
\left(\mathrm{~km} \mathrm{~s}^{-1}\right)\end{array}$ & $\begin{array}{l}T_{\mathrm{B}}^{\mathrm{a}} \\
(\mathrm{K}) \\
\end{array}$ & $\underset{\left(\mathrm{K} \mathrm{km} \mathrm{s}^{-1}\right)}{T_{\mathrm{B}} \mathrm{d} V}$ \\
\hline \multicolumn{5}{|c|}{$\mathrm{CH}_{3} \mathrm{CN}$} \\
\hline 0 & $47.8 \pm 0.2$ & $9.4 \pm 0.2$ & $9.1 \pm 1.5$ & $91 \pm 9$ \\
\hline 1 & " & " & $11.6 \pm 1.5$ & $116 \pm 8$ \\
\hline 2 & $"$ & $"$ & $10.9 \pm 1.5$ & $109 \pm 6$ \\
\hline 3 & $"$ & $"$ & $10.0 \pm 1.5$ & $100 \pm 6$ \\
\hline 4 & $"$ & $"$ & $8.7 \pm 1.5$ & $87 \pm 6$ \\
\hline 5 & $"$ & $9.4 \pm 0.2^{b}$ & $6.5 \pm 1.3$ & $67 \pm 9$ \\
\hline 6 & $"$ & " & $7.0 \pm 1.3$ & $70 \pm 9$ \\
\hline 7 & $"$ & $"$ & $3.0 \pm 1.3$ & $30 \pm 9$ \\
\hline 8 & $"$ & " & $2.2 \pm 1.3$ & $22 \pm 9$ \\
\hline 9 & $"$ & $"$ & $3.0 \pm 1.3$ & $28 \pm 9$ \\
\hline \multicolumn{5}{|c|}{$\mathrm{CH}_{3}{ }^{13} \mathrm{CN}^{c}$} \\
\hline 2 & $47.7 \pm 0.4$ & $9.4 \pm 0.2^{b}$ & $0.7 \pm 0.9$ & $7.3 \pm 8.7$ \\
\hline 3 & " & " & $1.5 \pm 0.9$ & $15.4 \pm 11$ \\
\hline 4 & $"$ & $"$ & $0.8 \pm 0.9$ & $7.5 \pm 8.7$ \\
\hline \multicolumn{5}{|c|}{$\mathrm{CH}_{3} \mathrm{CN} v_{8}=1$} \\
\hline$(2,1)$ & $46.8 \pm 0.5$ & $8.0 \pm 0.2^{b}$ & $1.9 \pm 0.4$ & $16.5 \pm 3.4$ \\
\hline$(3,1)$ & " & " & $1.8 \pm 0.4$ & $15.6 \pm 3.4$ \\
\hline$(4,1)$ & $"$ & $"$ & $2.2 \pm 0.4$ & $18.9 \pm 3.4$ \\
\hline$(1,-1)$ & $"$ & " & $1.8 \pm 0.4$ & $15.8 \pm 5.5$ \\
\hline$(0,1)$ & " & " & $2.3 \pm 0.4$ & $20.0 \pm 5.5$ \\
\hline$(2,-1)$ & " & " & $3.1 \pm 0.3$ & $26.3 \pm 4.5$ \\
\hline$(5,1)$ & " & $"$ & $1.5 \pm 0.3$ & $13.0 \pm 5.1$ \\
\hline$(3,-1)$ & " & " & $1.1 \pm 0.3$ & $9.6 \pm 3.5$ \\
\hline$(6,1)$ & " & " & $0.8 \pm 0.3$ & $7.1 \pm 3.5$ \\
\hline$(4,-1)$ & " & " & $2.1 \pm 0.8$ & $17.8 \pm 4.0$ \\
\hline$(7,1)$ & " & " & $1.5 \pm 0.7$ & $13.0 \pm 4.1$ \\
\hline$(5,-1)$ & " & " & $1.2 \pm 0.6$ & $10.0 \pm 3.4$ \\
\hline$(8,1)$ & $"$ & " & $0.2 \pm 0.4$ & $1.7 \pm 3.2$ \\
\hline$(1,1)$ & " & " & $1.8 \pm 0.2$ & $15.4 \pm 4.0$ \\
\hline$(6,-1)$ & " & " & $0.4 \pm 0.2$ & $3.5 \pm 4.1$ \\
\hline
\end{tabular}

Notes. ${ }^{(a)}$ Brightness temperature integrated over a $3 \sigma$ emission level area. ${ }^{(b)}$ This parameter was held fixed in the fit. ${ }^{\left({ }^{c}\right)} K=0$ and 1 are strongly blended with $\mathrm{CH}_{3} \mathrm{CN} K=5$, and it has not been possible to estimate their parameters.

of $\sim 50 \mathrm{~K}$ and methanol column densities ranging from $1 \times 10^{15}$ to $2.0 \times 10^{16} \mathrm{~cm}^{-2}$.

The typical deconvolved average angular diameter of the $50 \%$ contour level of the line emission of the hot core toward core A is $\sim 0$.' 4 , which corresponds to a linear diameter of $\sim 1300 \mathrm{AU}$, consistent with the size estimated from the dust emission (Table 3). Figure 12 shows a plot of the deconvolved size of the $50 \%$ contour level of the integrated emission of different $\mathrm{CH}_{3} \mathrm{CN}, \mathrm{CH}_{3}{ }^{13} \mathrm{CN}$, and $\mathrm{CH}_{3} \mathrm{CN} v_{8}=1$ transitions, and of the different species shown in Figs. 9 and 10. The higher the excitation energy of the transition, the more compact is the emission, in particular for $\mathrm{CH}_{3} \mathrm{CN}$. Taking only $\mathrm{CH}_{3} \mathrm{CN}$ and $\mathrm{CH}_{3} \mathrm{CN} v_{8}=1$ transitions into account, one finds that the deconvolved size of the $\mathrm{CH}_{3} \mathrm{CN} K=2$ component $\left(E_{\mathrm{u}}=196 \mathrm{~K}\right)$, which is 0.38 $(\sim 1200 \mathrm{AU})$, is more than twice the size of the $K, l=(6,-1)$ transition $\left(E_{\mathrm{u}}=1030 \mathrm{~K}\right)$, which is 0.15 (480 AU). This suggests a high opacity in the core and/or the existence of a temperature gradient, with the temperature increasing toward the center of the core, which would be consistent with the existence of an embedded YSO.

The masses of the individual cores, their $\mathrm{H}_{2}$ column densities, $N_{\mathrm{H}_{2}}$, and their $\mathrm{H}_{2}$ volume densities, $n_{\mathrm{H}_{2}}$, which are given

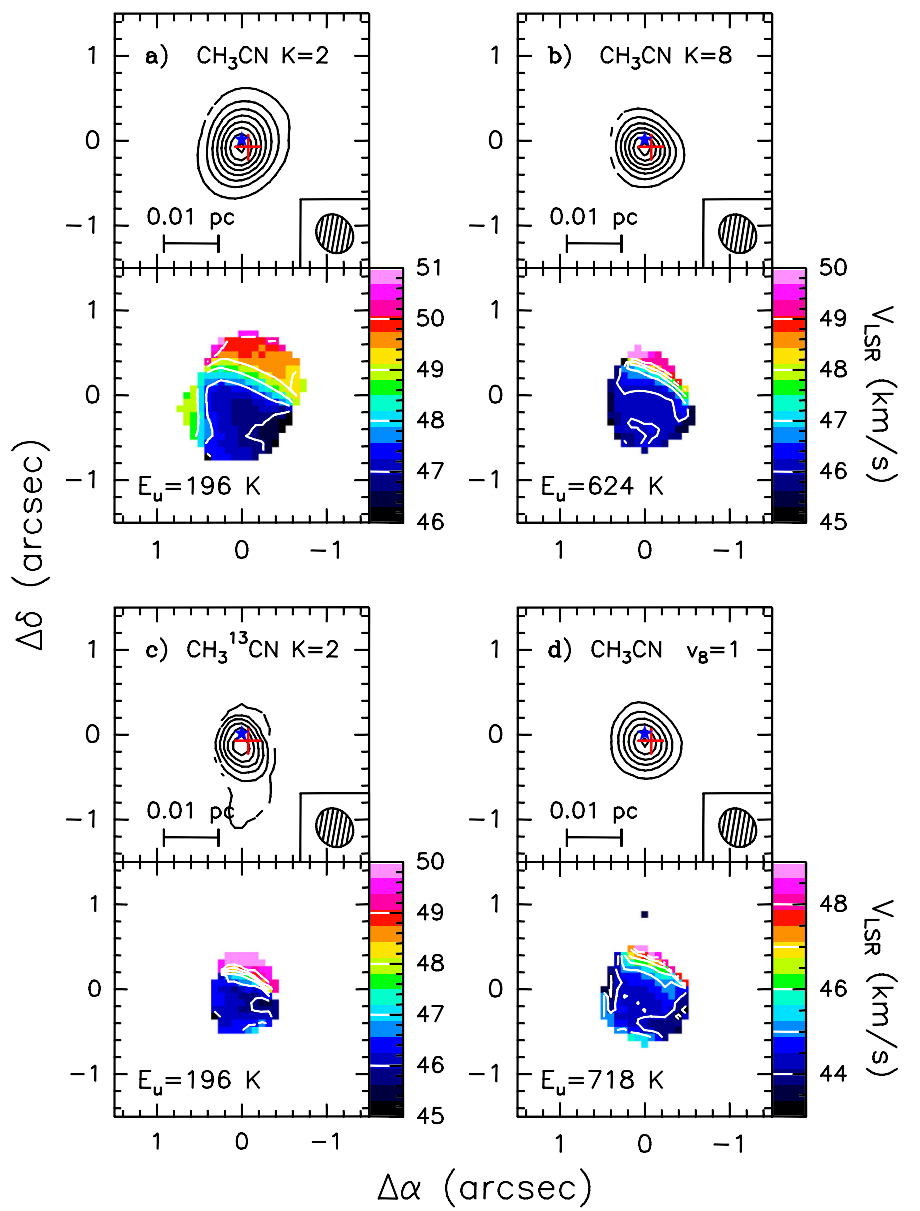

Fig. 8. Maps of the integrated intensity (moment 0) (upper panels) and the line velocity (moment 1) (lower panels) toward core A of a) $\mathrm{CH}_{3} \mathrm{CN} K=2$, b) $\mathrm{CH}_{3} \mathrm{CN} K=8$, c) $\mathrm{CH}_{3}^{13} \mathrm{CN} K=2$, and d) $\mathrm{CH}_{3} \mathrm{CN}$ $K, l=(3,1) v_{8}=1$ toward the HMC A in G35.03. Contour levels, which start at $3 \sigma$, are a) 0.3 to 4.5 by $0.6 \mathrm{Jy} / \mathrm{beam} \mathrm{km} \mathrm{s}^{-1}$, b) 0.1 to 1.3 by $0.2 \mathrm{Jy} /$ beam $\mathrm{km} \mathrm{s}^{-1}$, c) 0.03 to 0.33 by $0.06 \mathrm{Jy} / \mathrm{beam} \mathrm{km} \mathrm{s}{ }^{-1}$, and d) 0.1 to 1.1 by $0.2 \mathrm{Jy} / \mathrm{beam} \mathrm{km} \mathrm{s}^{-1}$. Offsets are measured with respect to the phase center. The red cross indicates the position of the dust emission peak of core A. The blue star indicates the position of the compact centimeter source CM2 detected by Cyganowski et al. (2011). The ALMA synthesized beam is shown in the lower right corner (upper panels). The energy of the upper level of each transition is indicated in the lower left corner (lower panels).

in Table 5, were estimated from the $870 \mu \mathrm{m}$ dust continuum emission assuming a dust opacity of $1.75 \mathrm{~cm}^{2} \mathrm{~g}^{-1}$ at $870 \mu \mathrm{m}$ (Ossenkopf \& Henning 1994) and a gas-to-dust ratio of 100. The dust temperature, $T_{\text {dust }}$, of the cores was assumed to be equal to the $T_{\text {ex }}$ estimated for $\mathrm{CH}_{3} \mathrm{OH}$. The masses of the cores are $\sim 1-$ $5 M_{\odot}$ (Table 5). These masses are low enough to suggest that such cores might be forming low-mass stars. While this could certainly be the case for most of the cores, it is less likely for cores A and D, because these two are associated with the (putative) HII regions CM1 and CM2 and present emission of hot-core species such as $\mathrm{CH}_{3} \mathrm{CN}$. These are typical signposts of highmass star formation.

A possible explanation for such low core masses could be that the interferometer is filtering out part of the continuum emission and that the dust emission observed would only trace the most compact component of the core. In fact, as seen in Sect. 3.1, the continuum ALMA Cycle 0 observations only recover $20 \%$ of the total flux of the compact core. On the other hand, most of the cores material might have already been destroyed by ionizing 
Table 5. Parameters of the cores.

\begin{tabular}{|c|c|c|c|c|c|c|c|c|c|}
\hline Core & $\begin{array}{l}T_{\text {ex }}{ }^{a} \\
(\mathrm{~K})\end{array}$ & $\log \left(N_{\mathrm{CH}_{3} \mathrm{OH}} / \mathrm{cm}^{-2}\right)$ & $\begin{array}{c}M_{\mathrm{gas}} b \\
\left(M_{\odot}\right) \\
\end{array}$ & $\begin{array}{c}N_{\mathrm{H}_{2}}{ }^{c} \\
\left(\times 10^{24} \mathrm{~cm}^{-2}\right) \\
\end{array}$ & $\begin{array}{c}n_{\mathrm{H}_{2}}{ }^{c} \\
\left(\times 10^{7} \mathrm{~cm}^{-3}\right) \\
\end{array}$ & $\begin{array}{c}\sum^{d} \\
\left(\mathrm{~g} \mathrm{~cm}^{-2}\right)\end{array}$ & $\begin{array}{c}\Delta V^{e} \\
\left(\mathrm{~km} \mathrm{~s}^{-1}\right)\end{array}$ & $\begin{array}{c}M_{\text {virial }} \\
\left(M_{\odot}\right) \\
\end{array}$ & $M_{\text {virial }} / M_{\mathrm{gas}}$ \\
\hline A & $240 \pm 30$ & $19.5 \pm 1.1$ & $0.75 \pm 0.13$ & $1.3 \pm 0.5$ & $9.9 \pm 5.3$ & $4.9 \pm 2.1$ & $6.0 \pm 0.8$ & $11 \pm 6^{f}$ & $14 \pm 10$ \\
\hline B & & & & & & & & & $>1.5$ \\
\hline $\mathrm{C}$ & $35 \pm 4$ & & $4.2 \pm 0.7$ & & $3.3 \pm$ & $4.2 \pm$ & $3.0 \pm$ & $15 \pm 9$ & $3.6 \pm 2.8$ \\
\hline $\mathrm{D}$ & $110 \pm 5$ & $17.7 \pm 0.5$ & $2.6 \pm 0.2$ & $1.1 \pm 0.3$ & $4.4 \pm 1.8$ & $4.4 \pm 1.3$ & $7.0 \pm 0.8$ & $65 \pm 22^{g}$ & $25 \pm 10^{g}$ \\
\hline $\mathrm{E}$ & $50 \pm 5$ & $16.0 \pm 0.3$ & $4.7 \pm 0.7$ & $1.9 \pm 0.7$ & $6.9 \pm 3.2$ & $7.2 \pm 2.6$ & $2.2 \pm 0.5$ & $7 \pm 4$ & $1.4 \pm 0.6$ \\
\hline $\mathrm{F}$ & $40 \pm 4$ & $15.0 \pm 0.3$ & $5.4 \pm 0.8$ & $2.8 \pm 0.9$ & $12 \pm 5$ & $11 \pm 4$ & $>1.4$ & $>2$ & $>0.37$ \\
\hline
\end{tabular}

Notes. ${ }^{(a)}$ Methanol excitation temperature, $T_{\text {ex }}$, estimated from the source averaged $\mathrm{CH}_{3} \mathrm{OH}$ emission with the myXCLASS software (see Sect. 4.1). ${ }^{(b)}$ Mass estimated using a dust opacity of $1.75 \mathrm{~cm}^{2} \mathrm{~g}^{-1}$ at $870 \mu \mathrm{m}$ (see Sect. 4.1). ${ }^{(c)}$ Estimated from the continuum. The dust temperature, $T_{\text {dust }}$, corresponds to the excitation temperature, $T_{\text {ex }} \cdot{ }^{(d)}$ Core mass surface density derived as $\Sigma=M_{\text {gas }} / \pi R^{2}$, where the radius of the source $R$ was obtained from its size $\theta$ (Table 3 ), and following the expression $R=\theta / 2 d$, where $d$ is the distance to G35.03. ${ }^{(e)}$ Line width estimated from $\mathrm{CH}_{3} \mathrm{OH} .{ }^{(f)}$ The contribution of the internal rotation motions of core A has been subtracted from the $\mathrm{CH}_{3} \mathrm{OH}$ line width in the calculation of $M_{\text {virial }}$ (see Sect. 4.2). ${ }^{(g)} M_{\text {virial }}$ would be $5.3 M_{\odot}$ if using a line width of $2 \mathrm{~km} \mathrm{~s}^{-1}$, which is the velocity dispersion of the core-free filament emission (see Sect. 3.2.1 and Fig. 5), and $M_{\text {virial }} / M_{\text {gas }}$ would be 2.

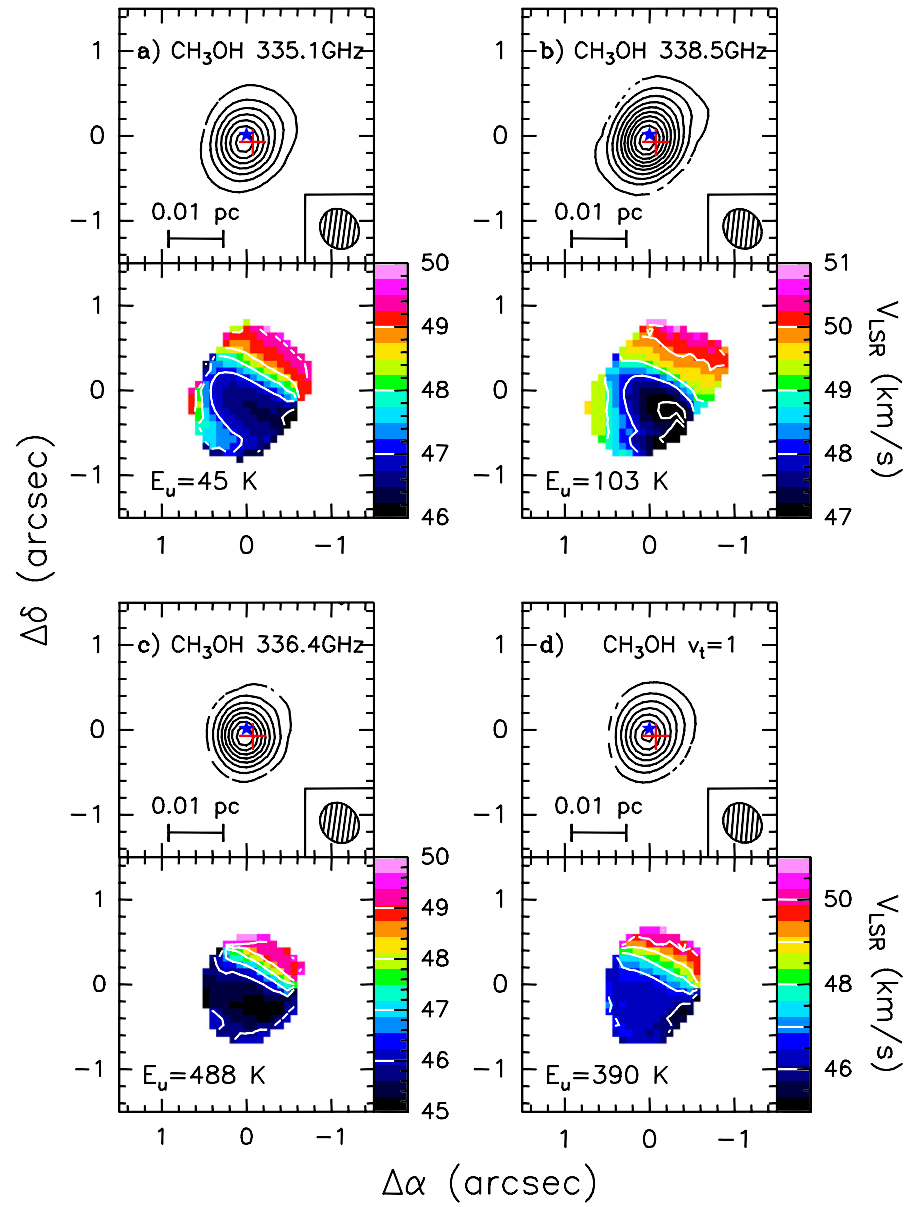

Fig. 9. Maps of the integrated intensity (moment 0) (upper panels) and the line velocity (moment 1) (lower panels) toward core A of a) $\mathrm{CH}_{3} \mathrm{OH}\left(2_{2,1}-3_{1,2}\right)$, b) $\mathrm{CH}_{3} \mathrm{OH}\left(7_{2,6}-6_{2,5}\right)$, c) $\mathrm{CH}_{3} \mathrm{OH}\left(14_{7,7}-15_{6,9}\right)$, and d) $\mathrm{CH}_{3} \mathrm{OH} v_{\mathrm{t}}=1\left(7_{1,7}-6_{1,6}\right)$ toward the HMC A in G35.03. Contour levels, which start at $3 \sigma$, are a) 0.2 to 2.6 by $0.4 \mathrm{Jy} /$ beam $\mathrm{km} \mathrm{s}^{-1}$, b) 0.3 to 5.7 by $0.6 \mathrm{Jy} /$ beam $\mathrm{km} \mathrm{s}^{-1}$, c) 0.1 to 1.5 by $0.2 \mathrm{Jy} / \mathrm{beam} \mathrm{km} \mathrm{s}{ }^{-1}$, and d) 0.2 to 2.6 by $0.4 \mathrm{Jy} / \mathrm{beam} \mathrm{km} \mathrm{s}^{-1}$. Offsets are measured with respect to the phase center. The symbols are the same as in Fig. 8.

photons and swept away by the bipolar flow powered by the embedded massive star(s). As a matter of fact, core masses of a few $M_{\odot}$ have been measured in other high-mass star-forming regions, for example IRAS 20126+4104, Cepheus A HW2,

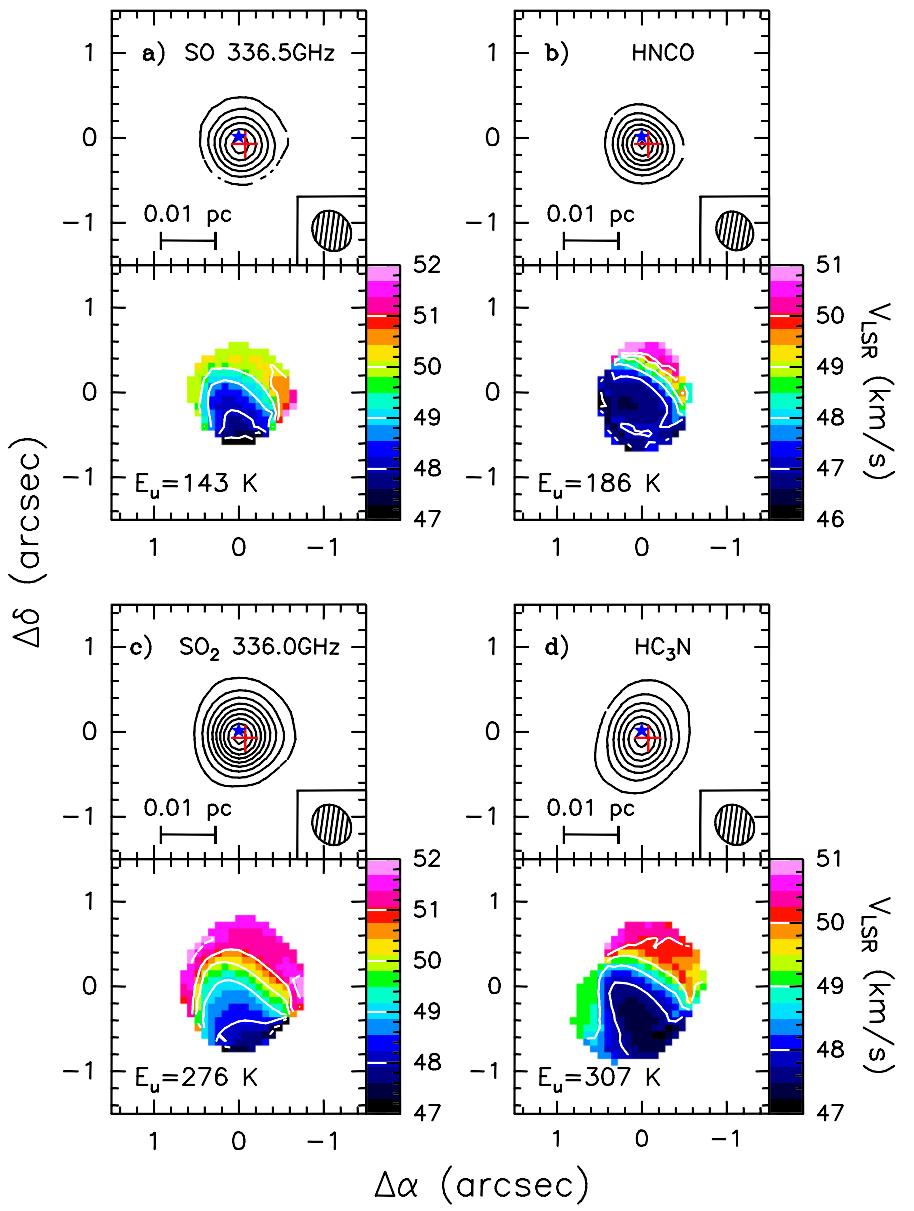

Fig. 10. Maps of the integrated intensity (moment 0 ) (upper panels) and the line velocity (moment 1) (lower panels) toward core A of a) SO $\left(10_{11}-10_{10}\right)$, b) HNCO $\left(16_{1,16}-15_{1,15}\right)$, c) $\mathrm{SO}_{2}\left(23_{3,21}-23_{2,22}\right)$, and d) $\mathrm{HC}_{3} \mathrm{~N}$ (37-36) toward the HMC A in G35.03. Contour levels, which start at $3 \sigma$, are 0.08 to 0.88 by $0.16 \mathrm{Jy} /$ beam $\mathrm{km} \mathrm{s}^{-1}$ b) 0.3 to 3.9 by $0.6 \mathrm{Jy} /$ beam $\mathrm{km} \mathrm{s}^{-1}$, c) 0.2 to 3.4 by $0.4 \mathrm{Jy} /$ beam km s${ }^{-1}$, and d) 0.3 to 3.9 by $0.6 \mathrm{Jy} /$ beam $\mathrm{km} \mathrm{s}^{-1}$. Offsets are measured with respect to the phase center. The symbols are the same as in Fig. 8.

AFGL 490, AFGL 2591, G35.20-0.74N (Cesaroni et al. 2007 and references therein), and more recently, G35.20-0.74N (Sánchez-Monge et al. 2013), and W3 IRS5 (Wang et al. 2013). 


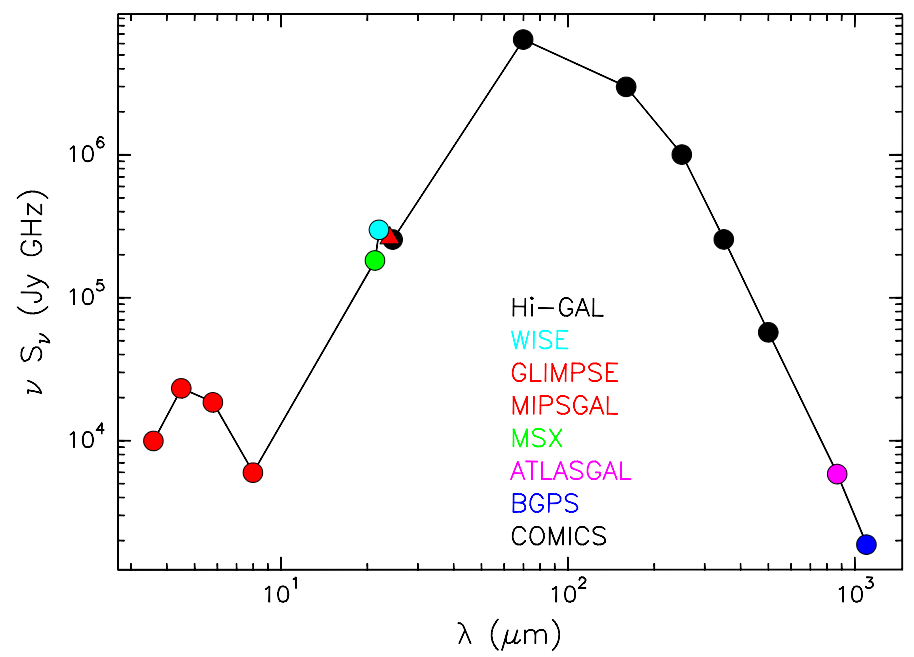

Fig. 11. Spectral energy distribution of the G35.03 star-forming region. Triangles denote lower limits. Different colors correspond to different instruments, as indicated in the figure.

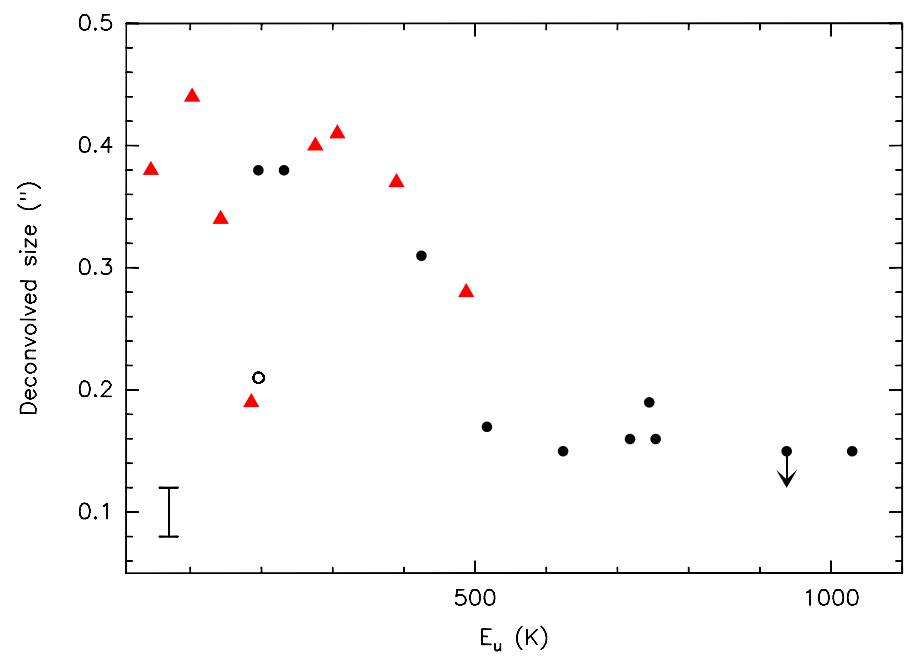

Fig. 12. Deconvolved size of the $50 \%$ contour level of the integrated emission of the different transitions shown in Figs. 8-10 versus upper level excitation energy. Black circles correspond to $\mathrm{CH}_{3} \mathrm{CN}$ and $\mathrm{CH}_{3} \mathrm{CN}$ $v_{8}=1$ transitions. The black open circle corresponds to the $K=2$ transition of the isotopologue of $\mathrm{CH}_{3} \mathrm{CN}$, that is, $\mathrm{CH}_{3}{ }^{13} \mathrm{CN}$. The black arrow indicates an upper limit for the deconvolved size because the emission in unresolved. The red triangles correspond to the other species shown in Figs. 9 and 10. The typical error bar of the size measurements is shown in the bottom left.

For IRAS $20126+4104$ and G35.20-0.74N, the cores turn out to be Keplerian disks rotating about B-type (proto)stars (Cesaroni et al. 2005; Sánchez-Monge et al. 2013). As discussed next, this could also be the case for core A in G35.03.

The $\mathrm{H}_{2}$ column densities, $N_{\mathrm{H}_{2}}$, estimated from the continuum are in the range $\sim 0.8 \times 10^{24}-3 \times 10^{24} \mathrm{~cm}^{-2}$. Comparing these values with $\mathrm{N}_{\mathrm{CH}_{3} \mathrm{OH}}$ (Table 5), the $\mathrm{CH}_{3} \mathrm{OH}$ abundances would be $2 \times 10^{-9}-2 \times 10^{-5}$. These abundances are consistent with the values found toward hot molecular cores and infrared dark clouds (Bisschop et al. 2007; Leurini et al. 2007).

\subsection{Stability of the filament and the cores}

Before studying the stability of the cores, we investigate the stability of the dusty U-shaped filament (Sect. 3.1). This filament has an integrated flux density of $1.3 \mathrm{Jy}$, which corresponds to a mass of the gas, $M_{\text {gas }}$, of 24-45 $M_{\odot}$ for a $T_{\text {dust }}$ range of $30-50 \mathrm{~K}$. For the sake of comparison, the mass of the gas calculated from the ATLASGAL $870 \mu \mathrm{m}$ peak flux of $5.8 \mathrm{Jy} \mathrm{beam}^{-1}$ is 106 $200 M_{\odot}$ for a $T_{\text {dust }}$ range of $30-50 \mathrm{~K}$. We modeled the filament as an infinite cylinder supported against its self-gravity by thermal and turbulent pressure, assumed to be spatially uniform. In this case, the filament has a critical mass per unit length beyond which no equilibrium can occur given by $\mathcal{M}_{\text {crit }}=2 \sigma^{2} / \mathrm{G}$, where $\sigma$ is the one-dimensional total (thermal plus turbulent) velocity dispersion and $G$ the gravitational constant (Ostriker 1964). The

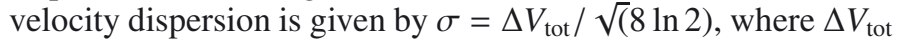
is the total (thermal plus turbulent) line width, which for the high-mass regime is close to the observed line width. If the filament is magnetized, the maximum mass per unit length can be higher than the value above. The simplest case is that of a magnetic field (axial or toroidal) with a uniform ratio of thermal-tomagnetic energy $\beta$. For a magnetic field in approximate equipartition with thermal energy, $\beta \sim 1$, the maximum mass per unit length is higher by a factor of a few.

Figure 5 shows that the velocity dispersion of the species tracing the extended continuum emission (Sect. 3.2.1) in the G35.03 filament is $\sim 2 \mathrm{~km} \mathrm{~s}^{-1}$, in the region not disturbed by the presence of submillimeter cores or centimeter sources. Therefore, we estimate $\mathcal{M}_{\text {crit }} \sim 335 M_{\odot} /$ pc. On the other hand, the total length of the G35.03 filament is $\sim 0.12 \mathrm{pc}$, and therefore the mass per unit length is $\mathcal{M}_{\text {line }}=200-375 M_{\odot} / \mathrm{pc}$. Since $\mathcal{M}_{\text {line }} \simeq \mathcal{M}_{\text {crit }}$, at this stage of the evolution, the filament in G35.03 seems to have reached a state of quasi-equilibrium resulting from the balance of self-gravity and turbulent pressure, even in the absence of magnetic fields. Taking into account that the ALMA interferometer is probably filtering out part of the extended continuum emission, then the estimated $\mathcal{M}_{\text {line }}$ should probably be considered a lower limit. On the other hand, because the ATLASGAL $870 \mu \mathrm{m}$ single-dish observations did not resolve the continuum emission toward G35.03, it is not possible to estimate the total length of the extended filament. Therefore, we can only estimate an upper limit for $\mathcal{M}_{\text {line }}$ by using the mass of the gas calculated from the ATLASGAL emission and assuming that the total length of the G35.03 filament is $\sim 0.12 \mathrm{pc}$. In this case, $\mathcal{M}_{\text {line }}$ would be $880-1670 M_{\odot} /$ pc. In case that the total length of the extended filament is $\sim 0.5 \mathrm{pc}$, then $\mathcal{M}_{\text {line }}$ should be comparable to $\mathcal{M}_{\text {crit }}$.

To investigate the stability of the cores, we estimated their virial masses, $M_{\text {virial }}$, by using the expression given by MacLaren et al. (1988), $M_{\text {virial }}=0.509 d \theta \Delta V^{2}$, where $d$ is the distance in kpc, $\theta$ is the size of the cores in arcsec, and $\Delta V$ is the line width in $\mathrm{km} \mathrm{s}^{-1}$. The virial mass depends on the density profile. For a power-law density distribution of the type $\rho \propto r^{-p}$, the virial mass in Table 5 should be multiplied by a factor $\alpha=3(5-2 p) / 5(3-p)$, which is $0.6 \leq \alpha \leq 1$ for $0 \leq p \leq 2$ (MacLaren et al. 1988). Thus, the values given in Table 5 should not be reduced by more than a factor 2 . To estimate $M_{\text {virial }}$, we used the size obtained from the continuum emission (Table 3) and the line width estimated from methanol. For core A, the line width estimated from $\mathrm{CH}_{3} \mathrm{OH}$ has a maximum contribution of $\sim 2 \mathrm{~km} \mathrm{~s}^{-1}$ (see Fig. 13) from the internal rotation motions. This contribution has been subtracted to estimate $M_{\text {virial }}$. The line width for cores B and F is a lower limit because the spectra show absorption, probably produced by the interferometer filtering out part of the extended emission. As seen in Table 5, despite the uncertainty on $M_{\text {virial }}$, the cores are, in general, subcritical $\left(M_{\text {virial }}>M_{\text {gas }}\right)$, in particular A and D. This suggests that the cores are unstable and should expand on timescales of the 


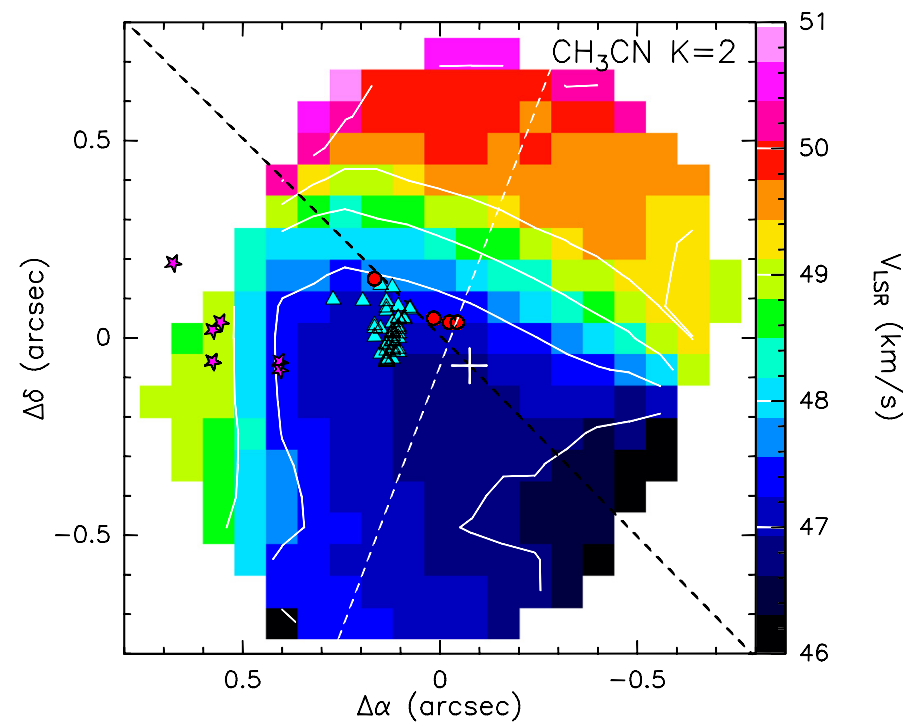

Fig. 13. Map of the line velocity (moment 1 ) of $\mathrm{CH}_{3} \mathrm{CN} K=2$ toward core A. Contour levels are the same as in Fig. 8. The white cross marks the position of the dust emission peak of core A. The dashed white line indicates the direction of the velocity gradient estimated as explained in Sect. 4.3. The dashed black line indicates the direction of the IR bipolar nebula (see Fig. 1). Red circles and magenta stars indicate the positions of $\mathrm{H}_{2} \mathrm{O}$ and $\mathrm{OH}$ masers, respectively (Forster \& Caswell 1999), and cyan triangles those of Class II $6.7 \mathrm{GHz} \mathrm{CH} \mathrm{CH}_{3} \mathrm{OH}$ masers (Cyganowski et al. 2009).

order of the sound crossing time, unless turbulence (probably injected from outside owing to instabilities associated with the winds rushing past) prevents it. Alternatively, ram pressure from external winds could confine the structures.

\subsection{Velocity gradients in core $A$}

The bottom panels of Figs. 8-10 show the line velocity (moment 1) maps of various lines of different molecular species toward core A. These have been selected to trace emission with upper level excitation energies ranging from 45 to $700 \mathrm{~K}$. As seen in these figures, all the species reveal a clear velocity gradient along the SE-NW direction independent of the excitation energy of the transition. For a given transition, the direction of the velocity gradient has been assumed to be the one passing through the peaks of the maps obtained by integrating the emission in the blue and red wings of the line. The corresponding PA ranges from $\sim-15^{\circ}$ to $\sim-30^{\circ}$. This is consistent with the major axis of the HMC obtained, after Gaussian deconvolution, with 2D-Gaussian fits to the integrated intensity maps of the most resolved species $\left(\sim-25^{\circ}\right.$ to $\left.\sim-35^{\circ}\right)$. This SE-NW direction is also consistent with the PA of $\sim-50^{\circ}$ of the major axis of the dust continuum emission, also estimated with 2D-Gaussian fitting to the source. The angle between the black and white dashed lines in Fig. 13 is $\sim 65^{\circ}$.

Given that the outflow axis is estimated using the wiggly IR nebula and considering possible inclination effects, this velocity gradient is roughly perpendicular to the direction of the NE-SW $4.5 \mu \mathrm{m}$ bipolar nebula (Figs. $2 \mathrm{a}$ and 13). Possible explanations for such a gradient could be expansion, infall, or rotation. The first can be ruled out because the velocity gradient should be oriented in the same direction as the molecular outflow, which is clearly not the case. Infall also seems impossible, because self-absorption would shift the peak velocity toward lower values at the core center, whereas we observe a steady velocity increase along a well-defined direction. That the velocity gradient is perpendicular to the direction of the NE-SW bipolar outflow (Fig. 13) strongly favors the rotation scenario, because this is exactly what is expected in the case of a disk rotating about the axis of the associated outflow. This scenario closely resembles the one for core B in the $3 \times 10^{4} L_{\odot}$ high-mass star-forming region G35.20-0.74N (see Sánchez-Monge et al. 2013), which is interpreted as a Keplerian disk rotating about the axis of a bipolar IR nebula. Finally, this velocity gradient might also be mimicked by two distinct cores with different $V_{\text {LSR }}$ that are too close to be resolved by our observations. In such a case, the velocity gradient would be a consequence of line emission from the two cores observed in the same instrumental beam. However, we believe this to be very unlikely and difficult to reconcile with the position-velocity (PV) cuts along the direction of the velocity gradient (Figs. 14, 15, 16, and 18) as we discuss below.

Figure 13 shows the line velocity (moment 1) map of $\mathrm{CH}_{3} \mathrm{CN} \mathrm{K}=2$ with the positions of the Class II $\mathrm{CH}_{3} \mathrm{OH}$ masers (Cyganowski et al. 2009) and $\mathrm{H}_{2} \mathrm{O}$ and $\mathrm{OH}$ masers (Forster \& Caswell 1999) as indicated. The methanol, water, and hydroxyl masers are all located in the blueshifted emission part of the core, with the $\mathrm{OH}$ masers located $\sim 0$ '. 4 eastward of the other maser positions. The $6.7 \mathrm{GHz}$ Class II $\mathrm{CH}_{3} \mathrm{OH}$ and $\mathrm{OH}$ maser emission is also blueshifted relative to the $V_{\mathrm{LSR}}$, with velocity shifts of $\sim 6-12 \mathrm{~km} \mathrm{~s}^{-1}$ for methanol and of $\sim 1-5 \mathrm{~km} \mathrm{~s}^{-1}$ for hydroxyl. This suggests that both Class $\mathrm{II} \mathrm{CH}_{3} \mathrm{OH}$ and $\mathrm{OH}$ maser emission could also be tracing the rotation of the core. On the other hand, the $\mathrm{H}_{2} \mathrm{O}$ masers are redshifted with respect to the $V_{\mathrm{LSR}}$ of the thermal gas by $\sim 13-17 \mathrm{~km} \mathrm{~s}^{-1}$. Because the $\mathrm{H}_{2} \mathrm{O}$ maser emission is collisionally pumped, it is more likely to trace outflows and/or shock-dominated regions. In such a scenario, the water masers would not be associated with the rotating disk but with the molecular outflow perpendicular to it. In fact, the velocity of the $\mathrm{H}_{2} \mathrm{O}$ maser spots, clearly dissociated from that of the $\mathrm{CH}_{3} \mathrm{OH}$ and $\mathrm{OH}$ maser emission by $>14 \mathrm{~km} \mathrm{~s}^{-1}$, indicates a different material origin. In conclusion, the various maser distributions and their velocity ranges indicate the complex kinematics of the material associated with core A.

To better study the kinematics of the gas around core A and investigate whether the velocity gradient could be consistent with Keplerian rotation, we calculated the PV cuts in the direction with $\mathrm{PA}=-45^{\circ}$, for $\mathrm{CH}_{3} \mathrm{CN} K=2$ and $8, \mathrm{CH}_{3}^{13} \mathrm{CN}$ $K=2$, and $\mathrm{CH}_{3} \mathrm{CN} v_{8}=1 K, l=3,1$, different transitions of $\mathrm{CH}_{3} \mathrm{OH}$ and $\mathrm{CH}_{3} \mathrm{OH} v_{t}=1$, and $\mathrm{SO}, \mathrm{HNCO}, \mathrm{SO}_{2}$, and $\mathrm{HC}_{3} \mathrm{~N}$ (Figs. 14-16). This direction is perpendicular to that of the bipolar nebula and is a compromise between the direction of the major axis of the dust continuum emission of core A and that of the velocity gradients for the different species. The PV plots show a "butterfly" pattern, which is especially evident for $\mathrm{CH}_{3} \mathrm{CN} K=2$, SO, $\mathrm{HC}_{3} \mathrm{~N}$, and $\mathrm{SO}_{2}$ (see Fig. 18). This pattern is characterized by the low-velocity "spurs" of emission at about \pm 0 .' 6 and $\pm 2 \mathrm{~km} \mathrm{~s}^{-1}$ (relative to the systemic velocity of $\sim 49.5 \mathrm{~km} \mathrm{~s}^{-1}$ ) and the high-velocity "spikes" close to the zero offset (corresponding to the position of the continuum peak). Such a "butterfly" shape is also visible for $\mathrm{CH}_{3} \mathrm{OH}\left(7_{2,6}-6_{2,5}\right)$, although in this case it is less clear because this transition is blended with another methanol line, $\mathrm{CH}_{3} \mathrm{OH}\left(7_{-4,4}-6_{-4,3}\right)$. These $\mathrm{PV}$ plots features are clear signatures of Keplerian rotation. The PV plots of a binary system with two distinct cores with different $V_{\text {LSR }}$ would not show the "spurs" and "spikes" visible in Fig. 18, unless each core had a Keplerian circumstellar disk oriented and rotating in such a way that it mimicked a single Keplerian disk. However, this seems to be too complicated and ad hoc an explanation. Therefore, the most plausible explanation 
M. T. Beltrán et al.: The high-mass star-forming region G35.03+0.35 imaged with ALMA

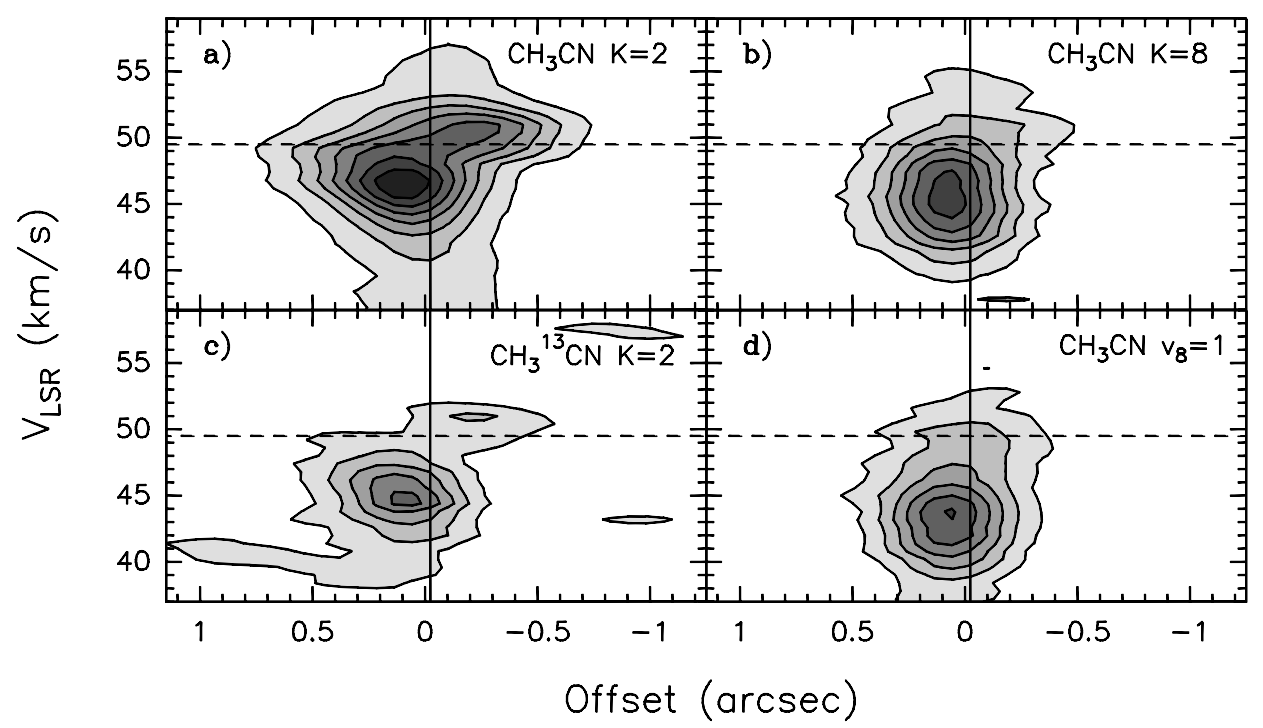

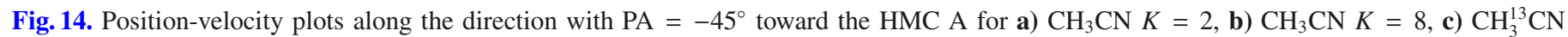
$K=2$, and d) $\mathrm{CH}_{3} \mathrm{CN} K, l=(3,1) v_{8}=1$. The offsets are measured from the phase center, positive to the southeast. Contour levels, which start at $3 \sigma$, are a) 0.03 to 0.39 by $0.06 \mathrm{Jy} \mathrm{beam}^{-1}$, b) 0.009 to 0.099 by $0.018 \mathrm{Jy}_{\text {beam }}^{-1}$, c) 0.006 to 0.055 by $0.012 \mathrm{Jy} \mathrm{beam}^{-1}$, and d) 0.01 to 0.09 by $0.015 \mathrm{Jy} \mathrm{beam}^{-1}$. The vertical solid line indicates the position of core A. The horizontal dashed line indicates the $V_{\mathrm{LSR}}$.

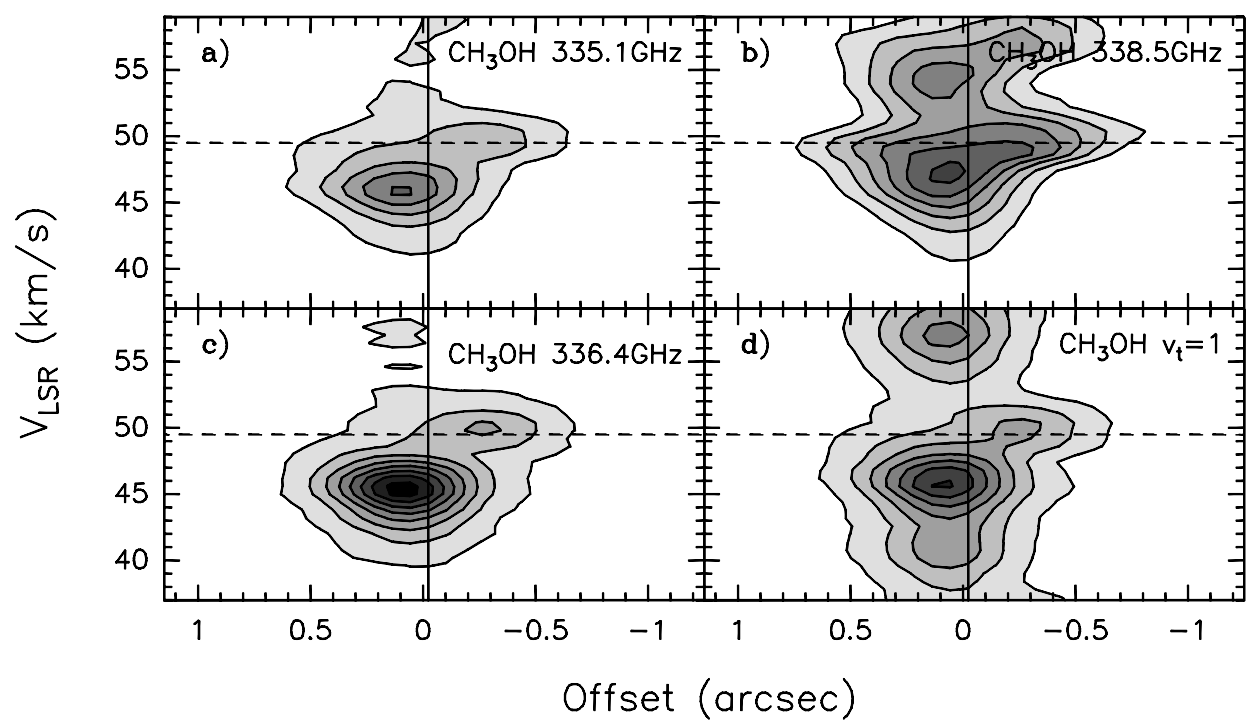

Fig. 15. Position-velocity plots along the direction with PA $=-45^{\circ}$ toward the $\mathrm{HMC}$ A for a) $\mathrm{CH}_{3} \mathrm{OH}\left(2_{2,1}-3_{1,2}\right)$, b) $\mathrm{CH}_{3} \mathrm{OH}\left(7_{2,6}-6_{2,5}\right)$, c) $\mathrm{CH}_{3} \mathrm{OH}\left(14_{7,7}-15_{6,9}\right)$, and d) $\mathrm{CH}_{3} \mathrm{OH} v_{t}=1\left(7_{1,7}-6_{1,6}\right)$. The methanol transition $\mathrm{CH}_{3} \mathrm{OH}\left(7_{2,6}-6_{2,5}\right)$ in panel b) is blended with another methanol line, $\mathrm{CH}_{3} \mathrm{OH}\left(7_{-4,4}-6_{-4,3}\right)$. The offsets are measured from the phase center, positive to the southeast. Contour levels, which start at $3 \sigma$, are a) 0.05 to 0.37 by $0.08 \mathrm{Jy} \mathrm{beam}^{-1}$, b) 0.09 to 0.54 by $0.09 \mathrm{Jy}_{\text {beam }}{ }^{-1}$, c) 0.01 to 0.22 by $0.03 \mathrm{Jy}_{\text {beam }}{ }^{-1}$, and d) 0.03 to 0.39 by $0.06 \mathrm{Jy} \mathrm{beam}^{-1}$. The vertical solid line indicates the position of core $\mathrm{A}$. The horizontal dashed line indicates the $V_{\mathrm{LSR}}$.

for the velocity gradient and the PV cuts in core A is that we are observing a Keplerian rotating disk. As clearly seen in the line velocity plots of the different tracers (Figs. 8-10) and the PV cuts (Figs. 14-16), the emission is lopsided, with much more emission to the blue and SE than to the red and NW. This indicates that the disk is asymmetric and non-homogeneous. This lack of uniformity has also been observed in other Keplerian disk candidates around B-type protostars, such as IRAS 20126+4104 (Cesaroni et al. 2014). Finally, the fact that, as seen in the PV plots, there is emission at positive offsets and redshifted velocities and at negative offsets and blueshifted velocities, i.e., outside the "butterfly" pattern, could indicate the presence of infall.
The PV plots in Fig. 14 show that the shift between the $V_{\mathrm{LSR}}$ and the velocity of the peak of the emission, $V_{\text {peak }}$, seems to increase with increasing excitation energy of the $\mathrm{CH}_{3} \mathrm{CN}$ and vibrationally excited transitions. To confirm this effect, we plot $\left|V_{\text {peak }}-V_{\text {sys }}\right|$, where $V_{\text {sys }}$ is the systemic velocity, as a function of the upper level excitation energy for different transitions of $\mathrm{CH}_{3} \mathrm{CN}$ and $\mathrm{CH}_{3} \mathrm{CN} v_{8}=1$ (Fig. 17). The transitions were selected to not be too blended with other transitions. The higher the energy of the transition, the higher the observed velocity. One expects this behavior for Keplerian rotation if the gas temperature and/or density increases when approaching the center of the disk. In this case, the high energy transitions, tracing gas 


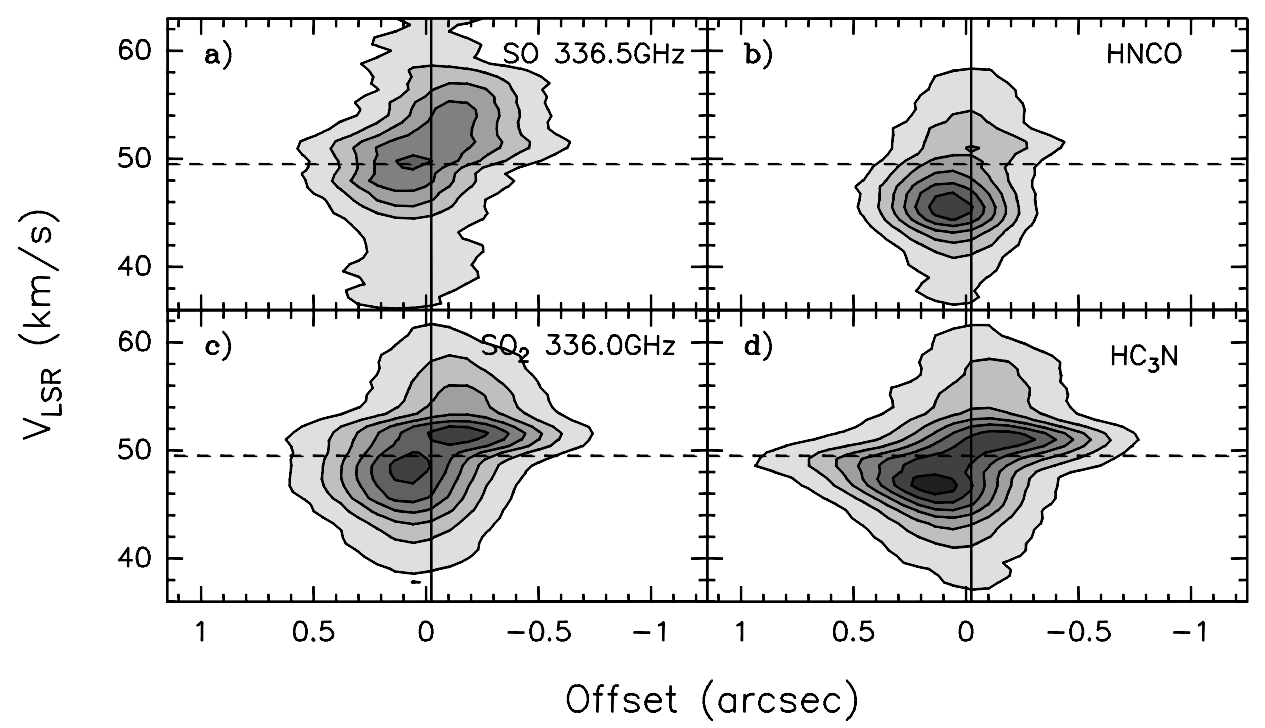

Fig. 16. Position-velocity plots along the direction with PA $=-45^{\circ}$ toward the HMC A for a) SO $\left(10_{11}-10_{10}\right)$, b) $\mathrm{HNCO}\left(16_{1,16}-15_{1,15}\right)$, c) $\mathrm{SO}_{2}\left(23_{3,21}-23_{2,22}\right)$, and d) $\mathrm{HC}_{3} \mathrm{~N}$ (37-36). The offsets are measured from the phase center, positive to the southeast. Contour levels, which start at $3 \sigma$, are a) 0.009 to 0.081 by $0.018 \mathrm{Jy} \mathrm{beam}^{-1}$, b) 0.04 to 0.24 by $0.04 \mathrm{Jy}_{\text {beam }}^{-1}$, c) 0.025 to 0.275 by $0.05 \mathrm{Jy}_{\text {beam }}^{-1}$, and d) 0.025 to 0.325 by $0.05 \mathrm{Jy} \mathrm{beam}^{-1}$. The vertical solid line indicates the position of core A. The horizontal dashed line indicates the $V_{\mathrm{LSR}}$.

close to the embedded protostar, are emitted with higher rotation velocities. Because infall produces a similar effect on the velocity field, we cannot rule out the possibility that the increase of the velocity toward the center is in part produced by infalling material.

In Fig. 18, we compare the PV plots for $\mathrm{CH}_{3} \mathrm{CN} K=2$, SO, $\mathrm{HC}_{3} \mathrm{~N}$, and $\mathrm{SO}_{2}$, with the patterns encompassing the region inside which emission is expected for an edge-on Keplerian disk of radius $0.43(\sim 0.007 \mathrm{pc})$ rotating about a star with a mass of $5 M_{\odot}$ and that of radius $0.75(\sim 0.012 \mathrm{pc})$ rotating about a star with a mass of $13 M_{\odot}$. These patterns agree reasonably well with the data, when considering that they are obtained for zero line width and infinite angular resolution. The mass of the central star, $M_{\star}$, has not been corrected for the inclination angle, $i$, of the disk with respect to the plane of the sky. The corrected mass is $M_{\star} / \sin ^{2} i$. The estimated range of values of $M_{\star}$ is consistent with the mass of a main-sequence star of spectral type B1 $\left(10.7 M_{\odot}\right)$ : i.e., the spectral type estimated for $\mathrm{CM} 2$, which is the centimeter continuum source associated with core A, from the $1.3 \mathrm{~cm}$ continuum emission assuming that it is optically thin (Codella et al. 2010) and following the tables of Davies et al. (2011) and Mottram et al. (2011). As already mentioned in Sect. 3.3, the stellar luminosity of $6.3 \times 10^{3} L_{\odot}$ estimated for a B1 main-sequence star like CM2 (Codella et al. 2010) is lower than the bolometric luminosity of $2 \times 10^{4} L_{\odot}$ computed from the SED for the whole star-forming region (see Sect. 3.3). However, the region over which the SED has been integrated also includes emission associated with the UC HII region CM1. This centimeter-continuum source has a $1.3 \mathrm{~cm}$ flux density that is consistent with a single ionizing star of spectral type B0.5 (Codella et al. 2010), corresponding to a stellar luminosity of $1.1 \times 10^{4} L_{\odot}($ Mottram et al. 2011).

The Keplerian pattern is less evident for transitions like $\mathrm{CH}_{3} \mathrm{CN} K=8, \mathrm{CH}_{3} \mathrm{CN} v_{8}=1 K, l=3,1$ or $\mathrm{HNCO}\left(16_{1,16^{-}}\right.$ $\left.15_{1,15}\right)$, which are only detected over a region close to the position of the star. One possible explanation for this different behavior could be the existence of a temperature gradient in the disk. In this scenario, the highest excitation transitions would only be excited in a small region closer to the central protostar

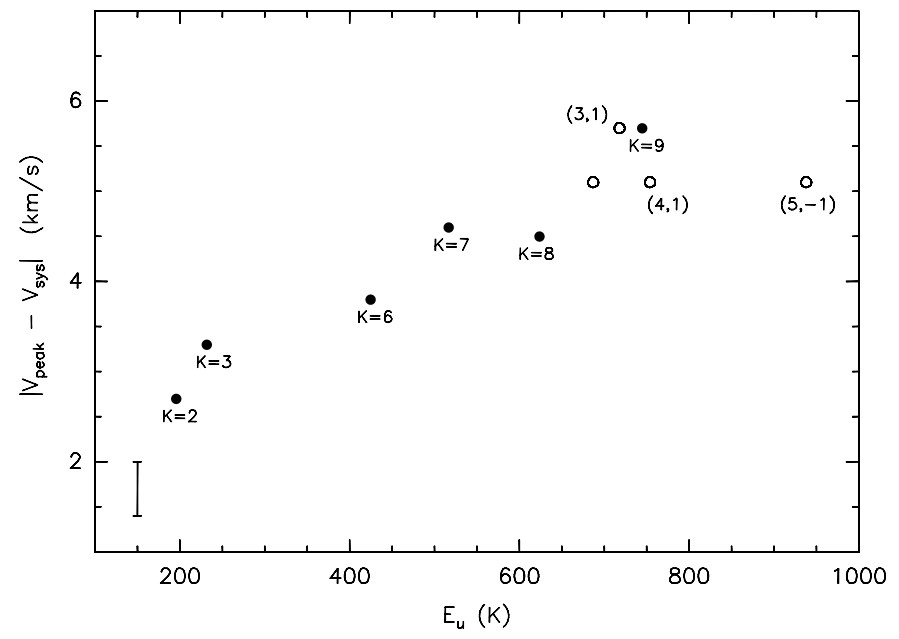

Fig. 17. Difference between the velocity of the peak of the emission in the $\mathrm{PV}$ plots along the direction with $\mathrm{PA}=-45^{\circ}$ toward core $\mathrm{A}$ and the systemic velocity for several transitions of $\mathrm{CH}_{3} \mathrm{CN}$ and $\mathrm{CH}_{3} \mathrm{CN} v_{8}=1$ as a function of upper level excitation energy. The systemic velocity $V_{\text {sys }}$ is $\sim 49.5 \mathrm{~km} \mathrm{~s}^{-1}$. The open circles correspond to the $v_{8}=1$ transitions. The different $K$ and $(K, l)$ transitions are indicated.

but at higher velocity than the lower energy lines. That $\mathrm{CH}_{3} \mathrm{CN}$ $K=8$ and $\mathrm{CH}_{3} \mathrm{CN} v_{8}=1 K, l=3,1$ have very high excitation energies $(>600 \mathrm{~K})$ and that $\left|V_{\text {peak }}-V_{\text {sys }}\right|$ increases with energy supports this hypothesis. On the other hand, the excitation energy of the HNCO $\left(16_{1,16}-15_{1,15}\right)$ line is $186 \mathrm{~K}$, similar to that of $\mathrm{CH}_{3} \mathrm{CN} \mathrm{K}=2$ and smaller than that of $\mathrm{HC}_{3} \mathrm{~N}$ (37-36). In this case, a possible variation in the molecular abundance of this species, hence of the line opacity, seems to be a more plausible explanation.

Beltrán et al. (2011a) made a comparative study of the stability of the rotating structures around B-type and O-type stars by plotting the ratio of the free-fall timescale $\left(t_{\mathrm{ff}}\right)$ to the rotational period ( $\left.t_{\text {rot }}\right)$ versus $M_{\text {gas }}$, where $t_{\mathrm{ff}}$ is proportional to the dynamical timescale needed to refresh the material of the rotating structure and $t_{\text {rot }}$ is the rotational period at the outer radius. Because $t_{\mathrm{ff}}$ has been estimated assuming spherical symmetry, the 


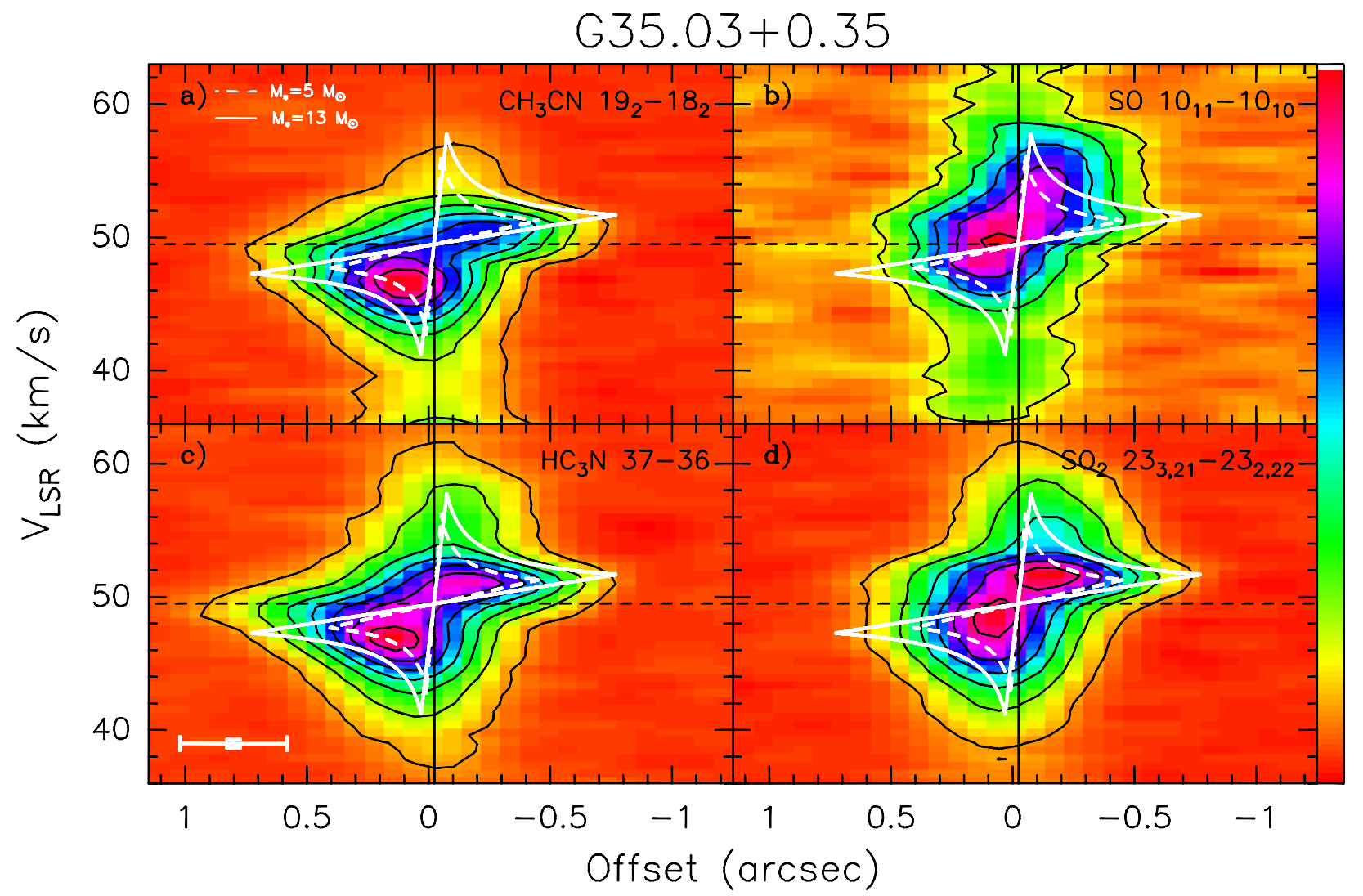

Fig. 18. Position-velocity plots along the direction with $\mathrm{PA}=-45^{\circ}$ toward the $\mathrm{HMC}$ A for a) $\mathrm{CH}_{3} \mathrm{CN} K=2$, b) $\mathrm{SO}\left(10_{11}-10_{10}\right)$, c) $\mathrm{HC} 3 \mathrm{~N}(37-$ $36)$, and d) $\mathrm{SO}_{2}\left(23_{3,21}-23_{2,22}\right)$. The thick white dashed and solid lines mark the border of the region where emission is expected for an edge-on Keplerian disk rotating about a $5 M_{\odot}$ and a $13 M_{\odot}$ point source, respectively. The offsets are measured from the phase center, positive to the southeast. Contour levels are the same as in Figs. 14 and 16. The vertical solid line indicates the position of core A. The horizontal dashed line indicates the $V_{\mathrm{LSR}}$. The error bars in the lower left-hand corner of panel c) indicate the angular and spectral resolution of the data.

$t_{\mathrm{ff}} / t_{\text {rot }}$ ratio for both disks and toroids would be overestimated. The correction factor, which depends on the scale height of the structures, would be higher when the rotating structures are flatter. However, we expect disks and toroids to be geometrically thick, unlike the very thin disks around low-mass objects such as, e.g., T Tauri stars, and therefore the correction factor to be small. For IRAS 20126+4104, which is the prototypical disk around a B-type star and one of the sources in the Beltrán et al. (2011a) comparative study, the hydrostatic scale height of the disk is $43 \%$ of the disk radius (Cesaroni et al. 2005, 2014), which reduces the free-fall time by only a factor 1.8. The conclusion of Beltrán et al. (2011a) is that disks around B-type stars and toroids around O-type stars occupy two distinct regions of the plot, with disks having a higher $t_{\mathrm{ff}} / t_{\mathrm{rot}}$ ratio and lower $M_{\mathrm{gas}}$.

To check whether this is also the case for core A in G35.03, we calculated $t_{\mathrm{ff}} / t_{\mathrm{rot}}$, for a rotation velocity $V_{\text {rot }}$ of $\sim 2 \mathrm{~km} \mathrm{~s}^{-1}$, which was estimated as half the velocity range measured in the gradients (see Figs. 8-10), and a radius $R \simeq 0.003 \mathrm{pc}$ $(\sim 600 \mathrm{AU})$, which is the typical deconvolved average radius of the $50 \%$ contour level of the line emission toward the HMC A, and a $M_{\text {gas }}$ of $0.75 M_{\odot}$ (Table 5). As shown in Fig. 19, G35.03 is consistent with the other Keplerian disk candidates found around B-type stars, in particular with the prototypical disk source IRAS 20126+4104 (Cesaroni et al. 2005; Beltrán et al. 2011a), which suggests that the structure rotates fast and that the accreting material can settle into a centrifugally supported disk. In Fig. 19, we also plotted the theoretical $t_{\mathrm{ff}} / t_{\text {rot }}$ curves for spherical clouds of mass $M_{\text {gas }}$ containing a star of mass $M_{\star}$ at the center, in which the gas is rotationally supported against the gravity of the gas plus the star. These curves can be expressed as $t_{\text {ff }} / t_{\text {rot }}=\left[\left(M_{\text {gas }}+M_{\star}\right) / 32 M_{\text {gas }}\right]^{1 / 2}$. The curves plotted in Fig. 19 correspond to $M_{\star}=0 M_{\odot}$ and $10 M_{\odot}$. As seen in the figure, the objects in the region of the toroids cannot be rotationally supported structures, unlike the objects located in the disks region that can indeed be rotationally supported. This strengthens the conclusion that disks and toroids are kinematically and dynamically different structures.

In conclusion, the structure observed toward core A in G35.03 seems to be consistent with a circumstellar disk in Keplerian rotation about a 5-13 $M_{\odot}$ star.

\subsection{Large-scale kinematics}

As already discussed in Sect. 3.2.1, the emission of some species, such as $\mathrm{H}^{13} \mathrm{CO}^{+}, \mathrm{C}_{2} \mathrm{H}, \mathrm{C}^{17} \mathrm{O}, \mathrm{H}_{2} \mathrm{CS}, \mathrm{C}^{34} \mathrm{~S}$, and $\mathrm{SiO}$, is extended and well correlated with the filamentary dust structure seen in the continuum at $343 \mathrm{GHz}$ (Fig. 5). Both the extended gas and dust emission seem to avoid a region located SW of core A and coincident with an IR emission peak (Fig. 20). The gas and dust emission from this region is so optically thin that it is not detected at submillimeter wavelengths, creating a cavity. This cavity is also visible in the PV plots along the direction with $\mathrm{PA}=+45^{\circ}$ for $\mathrm{H}^{13} \mathrm{CO}^{+}$and $\mathrm{C}^{34} \mathrm{~S}$ (see Fig. 21).

This cavity could be the result of a molecular outflow powered by the embedded protostar associated with core A that would have cleared the circumstellar material. Figure 5 shows the line velocity and the velocity dispersion maps of these 


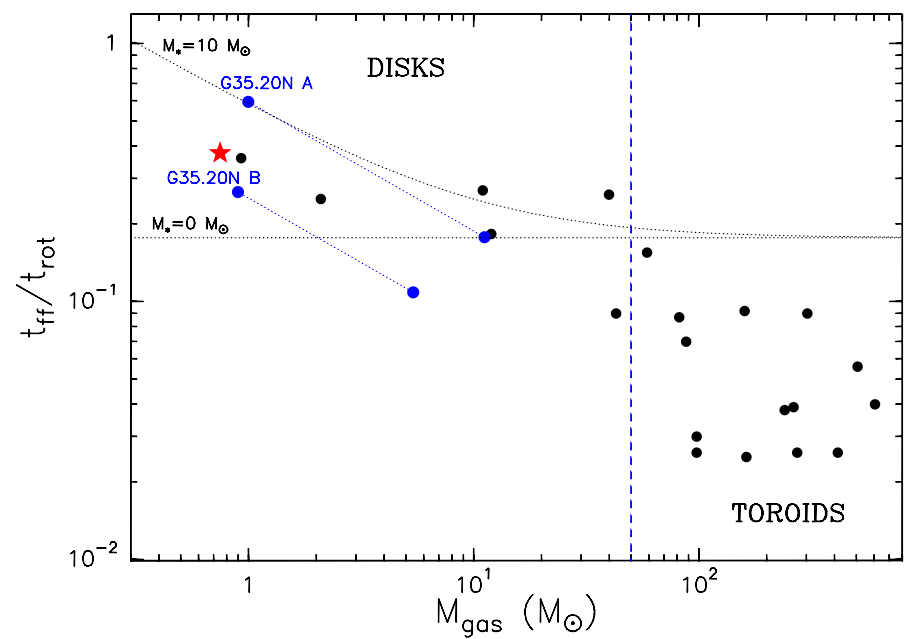

Fig. 19. Free-fall timescale to rotational period ratio, $t_{\mathrm{ff}} / t_{\text {rot }}$, versus gas mass, $M_{\text {gas }}$, of known rotating disks or toroids. Adapted from Fig. 12 of Beltrán et al. (2011a). The red star corresponds to core A in G35.03. Blue filled dots connected by a dotted line correspond to cores A and B in G35.20-0.74N, which occupy two different positions in the diagram depending on the value of the mass adopted (Sánchez-Monge et al. 2014). The parameters of the HMC G29.96-0.02 have been recalculated for a more correct distance of $6.2 \mathrm{kpc}$ (Russeil et al. 2011) instead of $3.5 \mathrm{kpc}$, which was the one used by Beltrán et al. (2011a). Black dotted lines correspond to the theoretical values of $t_{\mathrm{ff}} / t_{\text {rot }}$ for spherical clouds of mass $M_{\text {gas }}$ containing a star of mass $M_{\star}$ at the center, in which the gas is rotationally supported against the gravity of both the gas plus the star (see Sect. 4.3). The two dotted black lines correspond to $M_{\star}=0 M_{\odot}$ and $10 M_{\odot}$. The blue dashed line indicates a mass of $50 M_{\odot}$. The rotating structures with masses higher than this value are toroids.

extended species. The line velocity maps show a clear velocity gradient with blueshifted emission to the NE of core A and redshifted emission to the SW, consistent with the CO (3-2) integrated emission map obtained with the 10-m ASTE radio telescope (Paron et al. 2012). The velocity gradient is also visible in the PV plots (Fig. 21). The $V_{\mathrm{LSR}}$ of G35.03 is about $49.5 \mathrm{~km} \mathrm{~s}^{-1}$ (see Fig. 18), so apparently all this extended emission is moving at redshifted velocities with respect to $V_{\text {sys }}$ (see Fig. 21). This effect is particularly visible in Fig. 6, which shows that the $\mathrm{SiO}$ spectra at different positions in the core are all redshifted. This velocity gradient, including the range of velocities, is similar to the one detected in $\mathrm{NH}_{3}(1,1)$ with a much larger synthesized beam of 3". 5 by Brogan et al. (2011). As a result, the extended morphology, together with the NE-SW velocity gradient, would support the scenario in which we are observing a large-scale molecular outflow, whose direction would be coincident with that of the extended $4.5 \mu \mathrm{m}$ bipolar nebula, hence with that of the EGO. In this scenario, the IR emission peak visible SW of core A (Fig. 20) would be due to mid-infrared photons escaping through the cavity and produced by the embedded protostar that drives the outflow. This possibility has already been suggested by Cyganowski et al. (2011), who propose that the elongated MIPSGAL $24 \mu \mathrm{m}$ emission NE and SW of core A could be tracing warm dust in an outflow cavity. In this case, the almost symmetric location of the centimeter continuum sources $\mathrm{CM} 3$ and $\mathrm{CM} 4$ with respect to $\mathrm{CM} 2$ (core A) could indicate that these are part of a radio jet driven by the B-type protostar embedded in core A (Cyganowski et al. 2011). This situation would resemble that of IRAS 18162-2048 (HH 80-81: Martí et al. 1995) and IRAS 20126+4104 (Hofner et al. 1999), two high-mass protostars powering thermal radio jets. However, this

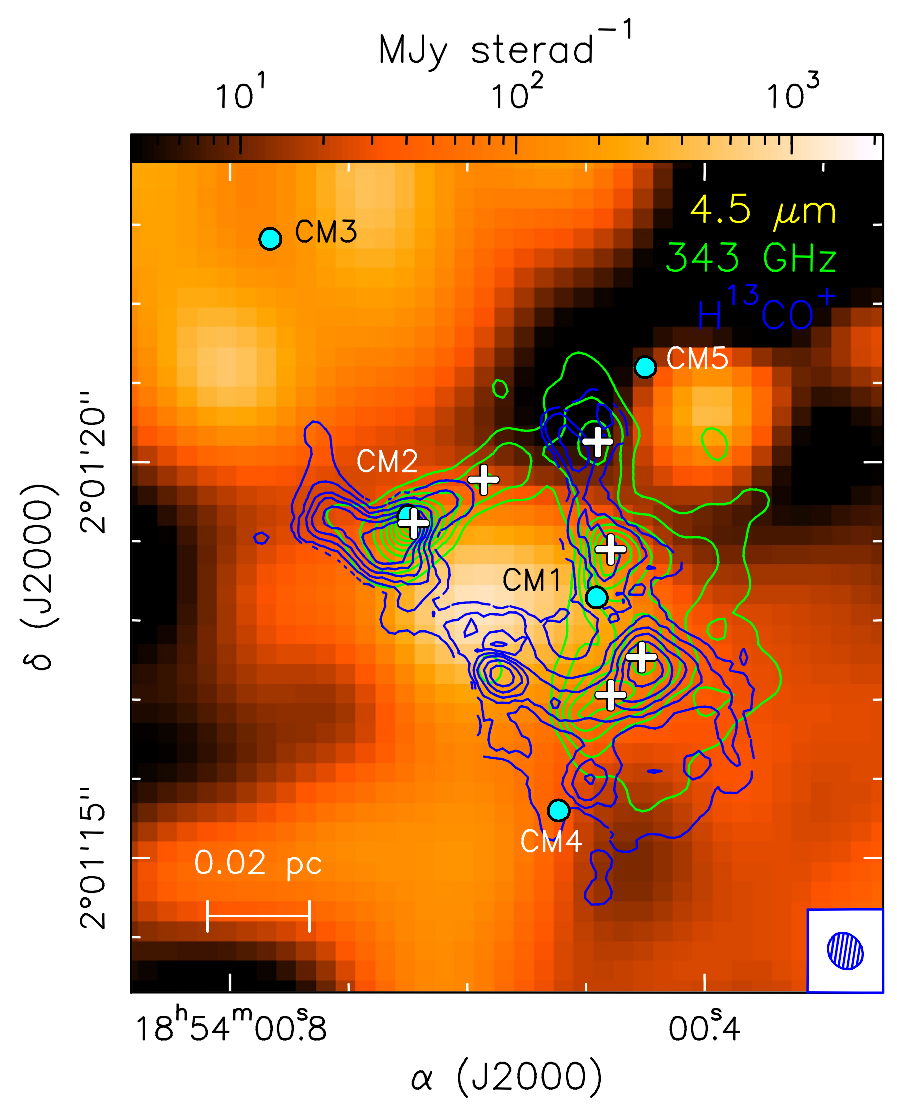

Fig. 20. Enhanced-resolution Spitzer $4.5 \mu \mathrm{m}$ image on logarithmic scale toward the star-forming region G35.03 overlaid with a contour map of the $343 \mathrm{GHz}$ continuum emission (green contours) and of the $\mathrm{H}^{13} \mathrm{CO}^{+}(3-2)$ (blue contours) integrated emission (moment 1) obtained with ALMA. The $\mathrm{H}^{13} \mathrm{CO}^{+}(3-2)$ emission has been integrated in the $[46,60] \mathrm{km} \mathrm{s}^{-1}$ velocity range. The submillimeter maps have been corrected for primary beam attenuation. Green contours are the same as in Fig. 2. The blue contours range from 105 to $840 \mathrm{mJy} \mathrm{beam}^{-1}$ in steps of $105 \mathrm{mJy}_{\text {beam }}{ }^{-1}$. The cyan circles indicate the positions of the 5 compact centimeter sources detected by Cyganowski et al. (2011), and the white crosses the positions of the submillimeter continuum cores (Table 3). The ALMA synthesized beam is shown in the lower right corner.

scenario presents a problem. Taking the geometry of the outflow into account, with blueshifted emission to the NE and redshifted to the SW, if the photons of the central protostar are escaping through the cavity open by the outflow, one would expect the brightest IR emission to the NE, unlike what is observed toward G35.03. In fact, the $2.2 \mu \mathrm{m} K$-band image from the UKIDSS survey $^{3}$ (Fig. 22) and the Spitzer $3.6 \mu \mathrm{m}$ image (Fig. A.1) show that the scattered emission from the near-infrared photons is indeed located toward the NE blueshifted lobe.

A more plausible explanation for the origin of the cavity is that it has been excavated by an expanding bubble created by the ionizing photons of an UC HII region. In this scenario, part of the extended material (Figs. 5 and 6) would be associated with the bipolar molecular outflow, and part could be material swept up by the ionization and shock fronts and, therefore, associated with the cavity, as the PV plots seem to suggest (Fig. 21).

3 The UKIDSS project is defined in Lawrence et al. (2007). UKIDSS uses the UKIRT Wide Field Camera (WFCAM; Casali et al. 2007). The photometric system is described in Hewett et al. (2006), and the calibration is described in Hodgkin et al. (2009). The pipeline processing and science archive are described in Hambly et al. (2008). 
M. T. Beltrán et al.: The high-mass star-forming region G35.03+0.35 imaged with ALMA

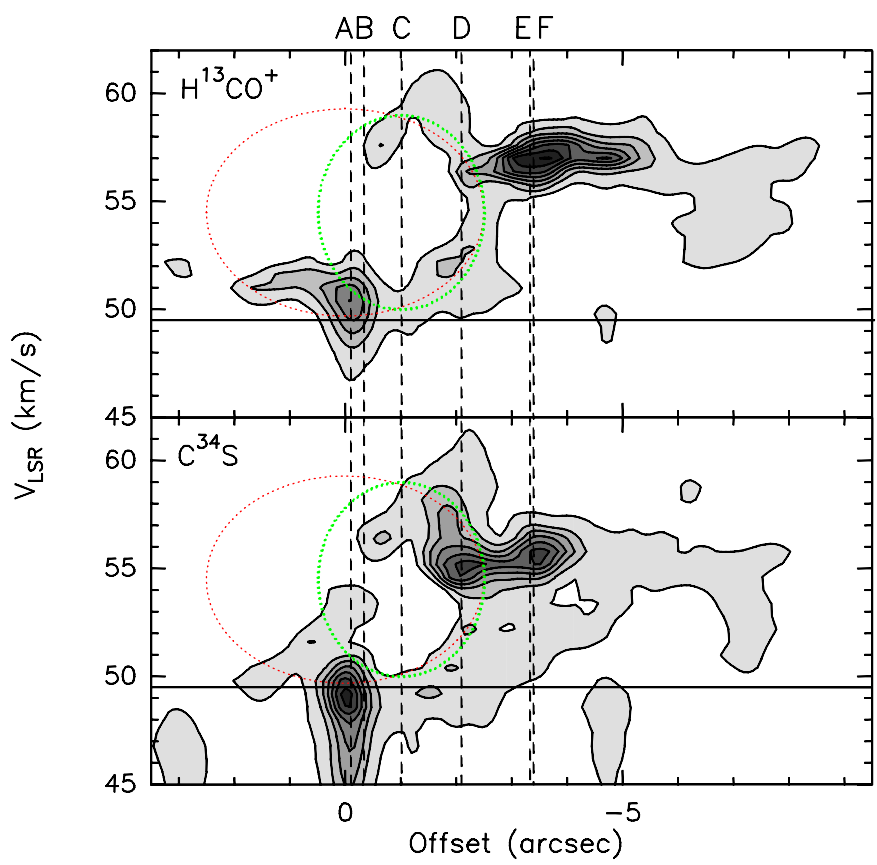

Fig. 21. Position-velocity plots along the direction with $\mathrm{PA}=+45^{\circ}$ for $\mathrm{H}^{13} \mathrm{CO}^{+}$and $\mathrm{C}^{34} \mathrm{~S}$. The offsets are measured from the phase center, positive to the northeast. The vertical dashed lines indicate the position of cores A, B, C, D, E, and F. The horizontal solid line indicates the $V_{\text {LSR }}$. The dotted ellipses outline two possibilities for explaining the morphology of the emission: a cavity centered on core A (red ellipse) or alternatively, a cavity centered close to the IR peak (green ellipse; see Sect. 4.4).

In fact, the green dotted ellipse in Fig. 21 outlines what could be a sort of expanding bubble. The two centimeter sources that could be powering this expanding bubble are the HC HII region $\mathrm{CM} 2$ and the UC HII region CM1, because they are the closest to the cavity. By taking a look at the PV plots (Fig. 21), none of these centimeter sources is located close to the center of the cavity. However, this could be a visual effect. Figure 23 shows the $870 \mu \mathrm{m}$ and the $8.4 \mathrm{GHz}$ (Cyganowski et al. 2011) continuum emission maps overlaid on the Spitzer $8.0 \mu \mathrm{m}$ image obtained with the HiRES deconvolution algorithm (Velusamy et al. 2008) and on the COMICS $25 \mu \mathrm{m}$ image. As seen in this figure, the centimeter continuum emission associated with the UC HII region CM1 is located closer to the SW IR peak than the submillimeter emission. In fact, the free-free emission associated with this UC HII region is very extended, with the low-level emission encompassing the bright IR emission peak. This suggests that CM1 could be, in fact, the driving source of the expanding bubble. This scenario could also explain the remarkable enhancement of the velocity dispersion seen south of core A in Fig. 5, which reaches a maximum value of $\sim 10 \mathrm{~km} \mathrm{~s}^{-1}$. In fact, this enhancement could be produced by the interaction of the redshifted material of the molecular outflow driven by core A and the material swept up by the ionization and shock fronts of the expanding UC HII region.

\section{Conclusions}

We carried out ALMA Cycle 0 observations at $870 \mu \mathrm{m}$ toward the high-mass star-forming region G35.03, which is associated with a NE-SW IR bipolar nebula. The continuum and the line emission of several species were observed with an angular

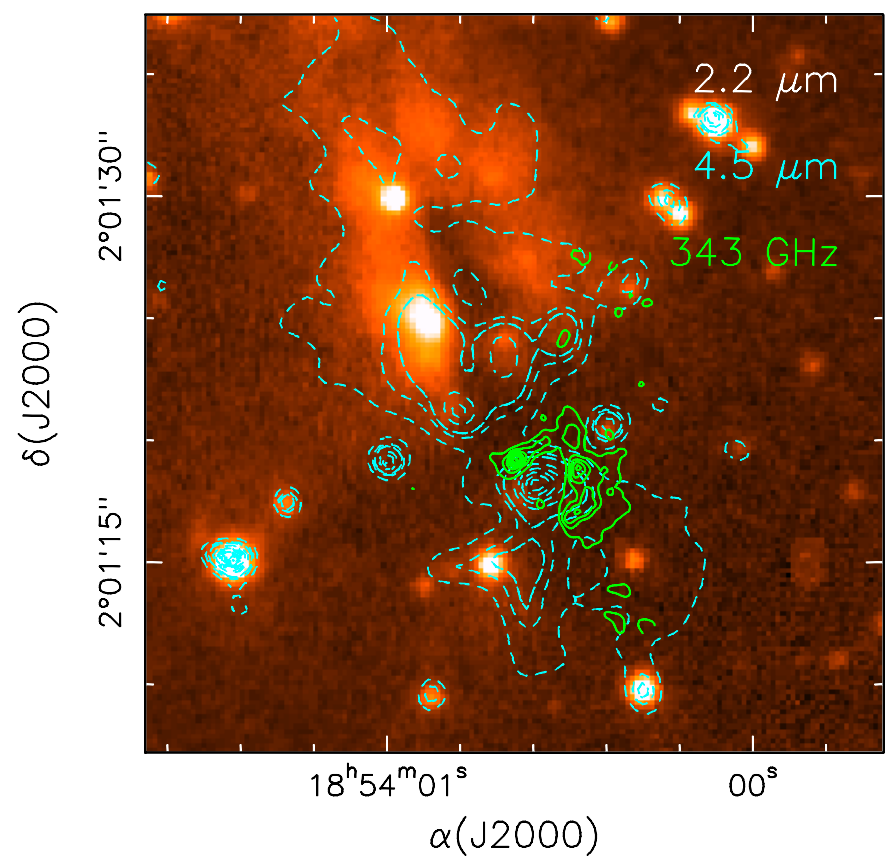

Fig. 22. 2.2 $\mu \mathrm{m} K$-band image toward G35.03 from the UKIDSS survey overlaid with a contour map of the enhanced resolution Spitzer $4.5 \mu \mathrm{m}$ image (cyan contours) and of the $343 \mathrm{GHz}$ continuum emission (green contours). Green contours are the same as in Fig. 2. Only the submillimeter continuum emission inside the ALMA primary beam has been taken into account.

resolution of $\sim 0$.' $^{\prime} 45$, which corresponds to $\sim 1400$ AU at the distance of the source.

The dust emission has revealed a filamentary U-shaped structure, which fragments into six cores, called A-F. Core A, which is the strongest at submillimeter wavelengths, lies at the center of the bipolar nebula and could be the driving source of the putative molecular outflow associated with the IR nebula. The temperature of the cores were estimated from $\mathrm{CH}_{3} \mathrm{OH}$ and are in the range of 35-50 K, except for cores A and D, which have temperatures of 240 and $110 \mathrm{~K}$, respectively. The latter cores are associated with compact centimeter sources and their free-free emission is consistent with that of a single ionizing star of spectral type B1 and B0.5, respectively. The masses of the cores are in the range $\sim 1-5 M_{\odot}$. Such low masses are probably the result of the interferometer filtering out $\sim 80 \%$ of the continuum emission.

The mass per unit length of the filament, $\mathcal{M}_{\text {line }} \sim$ 200-375 $M_{\odot} / \mathrm{pc}$, is similar to the critical mass of a thermally and turbulently supported infinite cylinder, $\mathcal{M}_{\text {crit }} \sim 335 M_{\odot} / p c$. This suggests that the filament can be in quasi-equilibrium even in the absence of magnetic fields. On the other hand, the cores are subcritical with $M_{\text {virial }}>M_{\text {gas }}$, which suggests that they are unstable and should expand unless turbulence and/or external ram pressure prevent it.

The emission of $\mathrm{H}^{13} \mathrm{CO}^{+}, \mathrm{C}_{2} \mathrm{H}, \mathrm{C}^{17} \mathrm{O}, \mathrm{C}^{34} \mathrm{~S}$, and $\mathrm{H}_{2} \mathrm{CS}$ is very extended and well correlated with the filamentary dust continuum emission, and it shows a NE-SW velocity gradient. Part of this extended material could be associated with the bipolar molecular outflow likely driven by core A. The gas emission seems to surround a mid-IR peak, which is clearly visible from 3.6 to $25 \mu \mathrm{m}$, and outlines a cavity. The most plausible explanation for the origin of this cavity is that it has been excavated by an expanding bubble created by the ionizing photons of the UC HII region CM1, which is associated with core D. 
A\&A 571, A52 (2014)
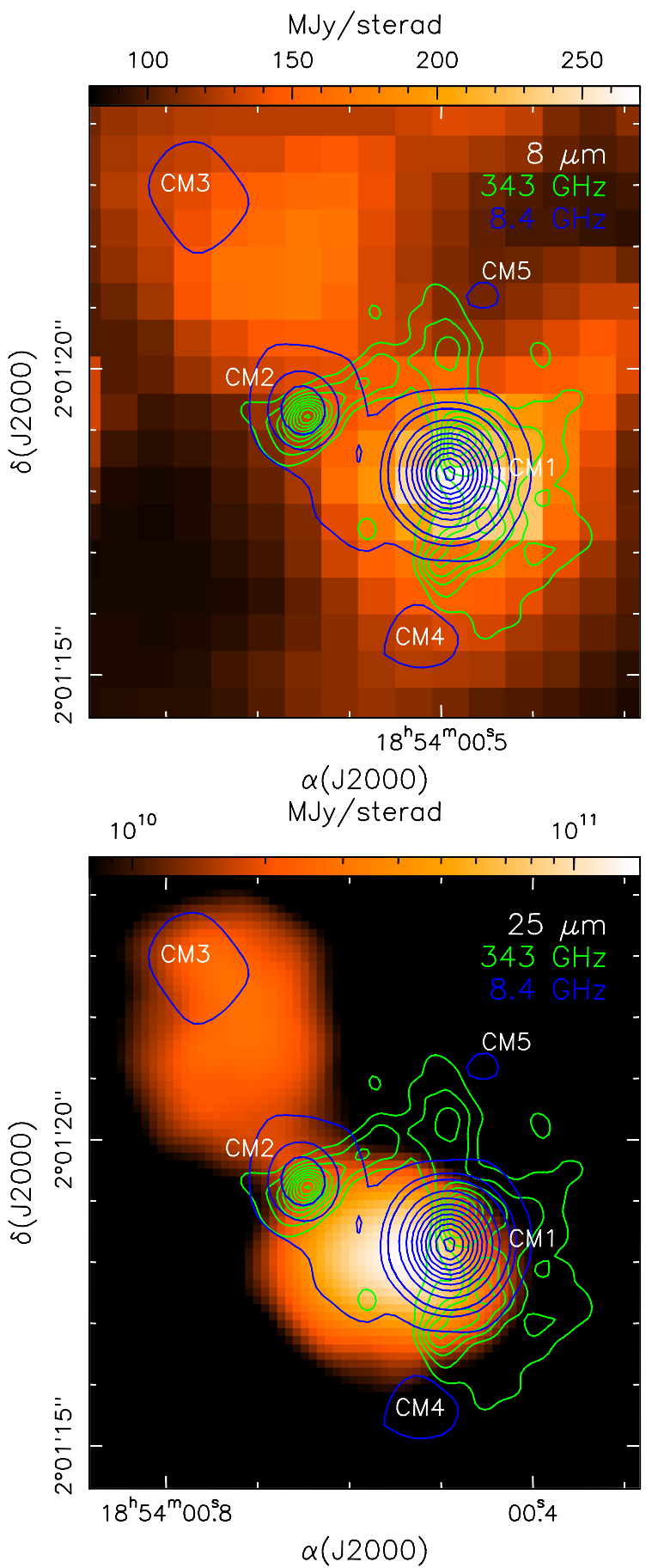

Fig. 23. Enhanced-resolution Spitzer $8.0 \mu \mathrm{m}$ image on linear scale (upper panel) and Subaru COMICS $25 \mu \mathrm{m}$ image in logarithmic scale (lower panel) toward the central part of G35.03 overlaid with a contour map of the $343 \mathrm{GHz}$ continuum emission (green contours) and of the $8.4 \mathrm{GHz}$ continuum emission (blue contours) from Cyganowski et al. (2011). The original COMICS $25 \mu \mathrm{m}$ image has been convolved with a Gaussian to obtain an angular resolution of $1^{\prime \prime}$. Green contours range from 8 to $108.8 \mathrm{mJy}_{\text {beam }}{ }^{-1}$ in steps of $14.4 \mathrm{mJy}_{\text {beam }}^{-1}$. Blue contours range from 0.108 to $0.918 \mathrm{mJybeam}^{-1}$ in steps of $0.405 \mathrm{mJy}^{-1}$ beam $^{-1}$, and from 0.918 to $11.853 \mathrm{mJy}^{-1}$ beam $^{-1}$ in steps of 1.215 mJy beam $^{-1}$.

The emission of typical hot core tracers, such as $\mathrm{CH}_{3} \mathrm{CN}$, has only been clearly detected toward core A and weakly toward core D. Core A is the most chemically rich source with strong emission of high excitation species and energies ranging up to $\sim 1000 \mathrm{~K}$. The emission of these high-density and highexcitation tracers is elongated in the SE-NW direction and shows a clear velocity gradient along this major axis of the core. That the velocity gradient is roughly perpendicular to the direction of the NE-SW bipolar outflow suggests that the emission is tracing a disk rotating about the axis of the associated outflow. The PV plots along the SE-NW direction of the velocity gradient show clear signatures of Keplerian rotation, although infall could also be present and are consistent with the pattern of an edge-on Keplerian disk rotating about a star with a mass in the range 5-13 $M_{\odot}$. The mass of the central star would agree with that of a main-sequence star of spectral type B1. The $t_{\text {ff }} / t_{\text {rot }}$ ratio for core A is consistent with that of the other Keplerian disk candidates found around B-type stars and is much higher than the values found toward rotating toroids around O-type stars. This high $t_{\mathrm{ff}} / t_{\text {rot }}$ ratio suggests that the structure rotates fast and that the accreting material has time to settle into a centrifugally supported disk. So far, together with G35.20-0.74N and the prototypical disk source IRAS 20126+4104 (Cesaroni et al. 2005), and maybe the sub-Keplerian disk candidate AFGL 2591-VLA3 (Wang et al. 2012), G35.03 is one of the most convincing examples of Keplerian disks rotating about high-mass protostars.

Acknowledgements. We thank Claudia Cyganowski for kindly providing the $8.4 \mathrm{GHz}$ continuum emission data of this region. This paper makes use of the following ALMA data: ADS/JAO.ALMA\#2011.0.00275.S. ALMA is a partnership of ESO (representing its member states), the NSF (USA), and NINS (Japan), together with NRC (Canada) and NSC and ASIAA (Taiwan), in cooperation with the Republic of Chile. The Joint ALMA Observatory is operated by ESO, AUI/NRAO, and NAOJ. We acknowledge the Italian ARC for the computational support. R.S.F. acknowledges T. Usuda, T. Inagaki, S. S. Hayashi, and H. Shinnaga for their help with the Subaru observations and data reduction. This work is based (in part) on archival data obtained with the Spitzer Space Telescope, which is operated by the Jet Propulsion Laboratory, California Institute of Technology under a contract with NASA. Support for this work was provided by an award issued by JPL/Caltech. The ATLASGAL project is a collaboration between the Max-Planck-Gesellschaft, the European Southern Observatory (ESO), and the Universidad de Chile. This publication makes use of data products from the Wide-field Infrared Survey Explorer, which is a joint project of the University of California Los Angeles, and the Jet Propulsion Laboratory/California Institute of Technology, funded by the National Aeronautics and Space Administration. This research made use of data products from the Midcourse Space Experiment. Processing of the data was funded by the Ballistic Missile Defense Organization with additional support from the NASA Office of Space Science. This research also made use of the NASA/ IPAC Infrared Science Archive, which is operated by the Jet Propulsion Laboratory, California Institute of Technology, under contract with NASA.

\section{References}

Anglada, G., Estalella, R., Pastor, J., Rodríguez, L. F., \& Haschick, A. D. 1996, ApJ, 463, p.205

Argon,. A. L., Reid, M. J., \& Menten, K. M. 2000, ApJS, 129, 159

Baudry, A., Desmurs, J. F., Wilson, T. L., \& Cohen, R. J. 1997, A\&A, 325, 255

Beltrán, M. T. 2011, in Computational Star Formation, eds. J. Alves, B. G. Elmegreen, J. M. Girart et al., Proc. IAU Symp., 270, 33

Beltrán, M. T., Cesaroni, R., Neri, R., Codella, C. et al. 2005, A\&A, 435, 901

Beltrán, M. T., Brand, J., Cesaroni, R., Fontani, F., Pezzuto, S. et al. 2006, A\&A, 447, 221

Beltrán, M. T., Cesaroni, R., Neri, R., \& Codella, C. 2011a, A\&A, 525, A151

Beltrán, M. T., Cesaroni, R., Zhang, Q., Galván-Madrid, R. et al. 2011b, A\&A 532, A91

Benjamin, R. A., Churchwell, E., Babler, B. L., et al. 2003, PASP, 115, 953

Bernst, I., Schilke, P., Moeller, T., et al. 2011, Astronomical Data Analysis Software and Systems XX, 442, 505

Bisschop, S. E., Jørgensen, J. K., van Dishoeck, E. F., \& de Wachter, E. B. M. 2007, A\&A, 465, 913

Blum, R. D., Damineli, A., \& Conti, P. S. 2001, AJ, 121, 3149

Bonnell, I. A., \& Bate, M. R. 2006, MNRAS, 370, 488

Bonnell, I. A., Bate, M. R., \& Zinnecker, H. 1998, MNRAS, 298, 93

Briggs, D. 1995, Ph.D. Thesis, New Mexico Inst. Mining \& Tech., USA

Brogan, C. L., Hunter, T. R., Cyganowski, C. J., et al. 2011, ApJ, 739, L16 
M. T. Beltrán et al.: The high-mass star-forming region G35.03+0.35 imaged with ALMA

Bronfman, L., Nyman, L-A., \& May, J. 1996, A\&AS, 115, 81

Burrows, C. J., Stapelfeldt, K. R., Watson, A. M., et al. 1996, ApJ, 473, 437

Carey, S. J., Noriega-Crespo, A., Mizuno, D. R., et al. 2009, PASP, 121, 76

Casali, M., Adamson, A., Alves de Oliveira, C., et al. 2007, A\&A, 467, 777

Cesaroni, R., Neri, R., Olmi, L., Testi, L. et al. 2005, A\&A, 434, 1039

Cesaroni, R., Galli, D., Lodato, G., Walmsley, C. M., \& Zhang, Q. 2007, in Protostars and Planets V, eds. B. Reipurth, D. Jewitt, \& K. Keil (University of Arizona Press), 197

Cesaroni, R., Galli, D., Neri, R., Walmsley, C. M. 2014, A\&A, 566, A73

Churchwell, E., Povich, M. S., Allen, D. et al. 2006, ApJ, 649, 759

Churchwell, E., Babler, B. L., Meade, M. R., et al. 2009, PASP, 121, 213

Codella, C., Cesaroni, R., López-Sepulcre, A., Beltrán, M. T., et al. 2010, A\&A, 510, A86

Cohen, M., Walker, R. G., Carter, B. et al. 1999, AJ, 117, 1864

Comito, C., Schilke, P., Phillips, T. G., et al. 2005, ApJS, 156, 1277

Cyganowski, C. J., Whitney, B. A., Holden, E., Braden, E., et al. 2008, AJ, 136, 2391

Cyganowski, C. J., Brogan, C. L., Hunter, T. R., \& Churchwell, E. 2009, ApJ, 702,1615

Cyganowski, C. J., Brogan, C. L., Hunter, T. R., \& Churchwell, E. 2011, ApJ, 743,56

Davies, B., Hoare, M. G., Lumsden, S. L. et al. 2011, MNRAS, 416, 972

Drosback, M. M., Aguirre, J., Bally, J., et al. 2008, BAAS, 40, 271

Forster, J. R., \& Caswell, J. L. 1989, A\&A, 213, 339

Forster, J. R., \& Caswell, J. L. 1999, A\&AS, 137, 43

Hambly, N. C., Collins, R. S., Cross, N. J. G. et al. 2008, MNRAS, 384, 637

Harju, J., Walmsley, C. M., \& Wouterloot, J. G. A. 1993, A\&AS, 98, 51

Hewett, P. C., Warren, S. J., Leggett, S. K., \& Hodgkin, S. T. 2006, MNRAS, 367,454

Hodgkin, S. T., Irwin, M. J., Hewett, P. C., \& Warren, S. J. 2009, MNRAS, 394, 675

Hofner, P., Cesaroni, R., Rodríguez, L. F., \& Martí, J. 1999, A\&A, 345, L43

Jijina, J., \& Adams, F. C. 1996, ApJ, 462, 874,

Kataza, H., Okamoto, Y., Takubo, S., et al. 2000, SPIE, 4008, 1144

Keto, E. 2007, ApJ, 666, 976

Krumholz, M. R., Klein, R. I., McKee, C. F., Offner, S. S. R., \& Cunningham, A. J. 2009, Science, 323, 754

Lanz, T., \& Hubeny, I. 2007, ApJS, 169, 83

Larionov, G. M., Val'tts, I. E., Winnberg, A., et al. 1999, A\&AS, 139, 257
Lawrence, A., Warren, S. J., Almaini, O. et al. 2007, MNRAS, 379, 1599

Leurini, S., Schilke, P., Wyrowski, F., \& Menten, K. M. 2007, A\&A, 466, 215

MacLaren, I., Richardson, K. Mn., \& Wolfendale, A. W. 1988, ApJ, 333, 821

Martí, J., Rodríguez, L. F., \& Reipurth, B. 1995, ApJ, 449, 184

Martins, F., Schaerer, D., \& Hillier, D. J. 2005, A\&A, 436, 1049

McKee, C. F., \& Tan, J. C. 2002, Nature, 416, 59

Menten, K. M. 1991, ApJ, 380. L75

Molinari, S., Swinyard, B., Bally, J., et al. 2010a, A\&A, 518, L100

Molinari, S., Swinyard, B., Bally, J., et al. 2010b, PASP, 122, 314

Möller, T., Bernst, I., Panoglou, D., et al. 2013, A\&A, 549, A21

Mottram, J. C., Hoare, M. G., Davies, B., Lumsden, S. L., et al. 2011, ApJ, 730, 33

Müller, H. S. P., Thorwirth, S., Roth, D. A., \& Winnewisser, G. 2001, A\&A, 370, L49

Nakano, T., Hasegawa, T., \& Norman, C. 1995, ApJ, 450, 183

Ossenkopf, V., \& Henning, Th. 1994, A\&A, 291, 943

Ostriker, J. 1964, ApJ, 140, 1056

Paron, S., Ortega, M. E., Petriella, A., et al. 2012, MNRAS, 419, 2206

Pickett, H. M., Poynter, R. L., Cohen, E. A., et al. 1998, J. Quant. Spectr. Rad. Transf., 60, 883

Price, S. D., Egan, M. P., \& Shipman, R. F. 1999, Astrophysics with Infrared Surveys: A Prelude to SIRTF, ASP Conf. Ser., 177, 394

Reid, M. J., Menten, K. M., Zheng, X. W., et al. 2009, ApJ, 700, 137

Russeil, D., Pestalozzi, M., Mottram, J. C., et al. 2011, A\&A, 526, A151

Sánchez-Monge, Á., Cesaroni, R., Beltrán, M. T., et al. 2013, A\&A, 552, L10

Sánchez-Monge, Á., Beltrán, M. T., Cesaroni, R., et al. 2014, A\&A, 569, A11

Schuller, F., Menten, K. M., Contreras, Y., et al. 2009, A\&A, 504, 415

Shepherd, D. S., \& Churchwell, E. 1996, ApJ, 457, 267

Schilke, P., Benford, D. J., Hunter, T. R., Lis, D. C., \& Phillips, T. G. 2001, ApJS, 132,281

Simon, M., Dutrey, A., \& Guilloteau, S. 2000, ApJ, 545, 1034

Velusamy, T., Marsh, K. A., Beichman, C. A., Backus, C. R., \& Thompson, T. J. 2008, AJ, 136, 197

Wang, K.-S., van der Tak, F. F. S., \& Hogerheijde, M. R. 2012, A\&A, 543, A 22

Wang, K.-S., Bourke, T. L., Hogerheijde, M. R., et al. 2013, A\&A, 558, A69

Wilson, T. L., \& Rood, R. T. 1994, ARA\&A, 86, 317

Wright, E. L., Eisenhardt, P. R. M., Mainzer, A. K., et al. 2010, AJ, 140, 1868

Zernickel, A., Schilke, P., Schmiedeke, A., et al. 2012, A\&A, 546, A87 


\section{Appendix A: IR images}

In Figs. A.1 and A.2, we show the IR and submillimeter images of G35.03 from the Spitzer GLIMPSE survey, Subaru COMICS, the Herschel Hi-GAL survey, and the CSO BGPS survey at representative wavelengths.

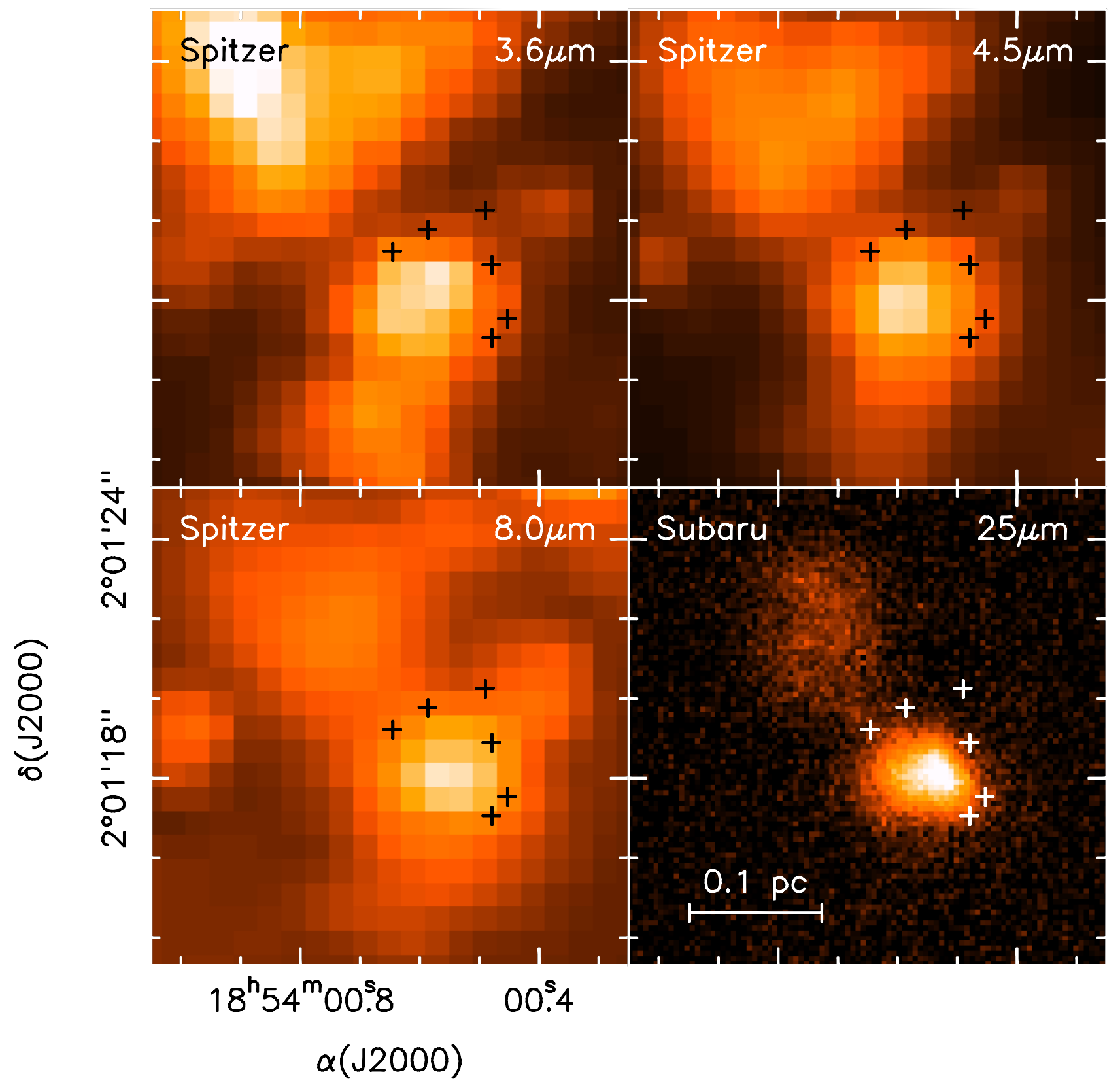

Fig. A.1. Spitzer 3.6, 4.5, and $8.0 \mu \mathrm{m}$ (from the GLIMPSE survey), and Subaru COMICS $25 \mu \mathrm{m}$ images on linear scale toward the star-forming region G35.03. The crosses indicate the positions of the submillimeter continuum cores (Table 3 ). 
M. T. Beltrán et al.: The high-mass star-forming region G35.03+0.35 imaged with ALMA

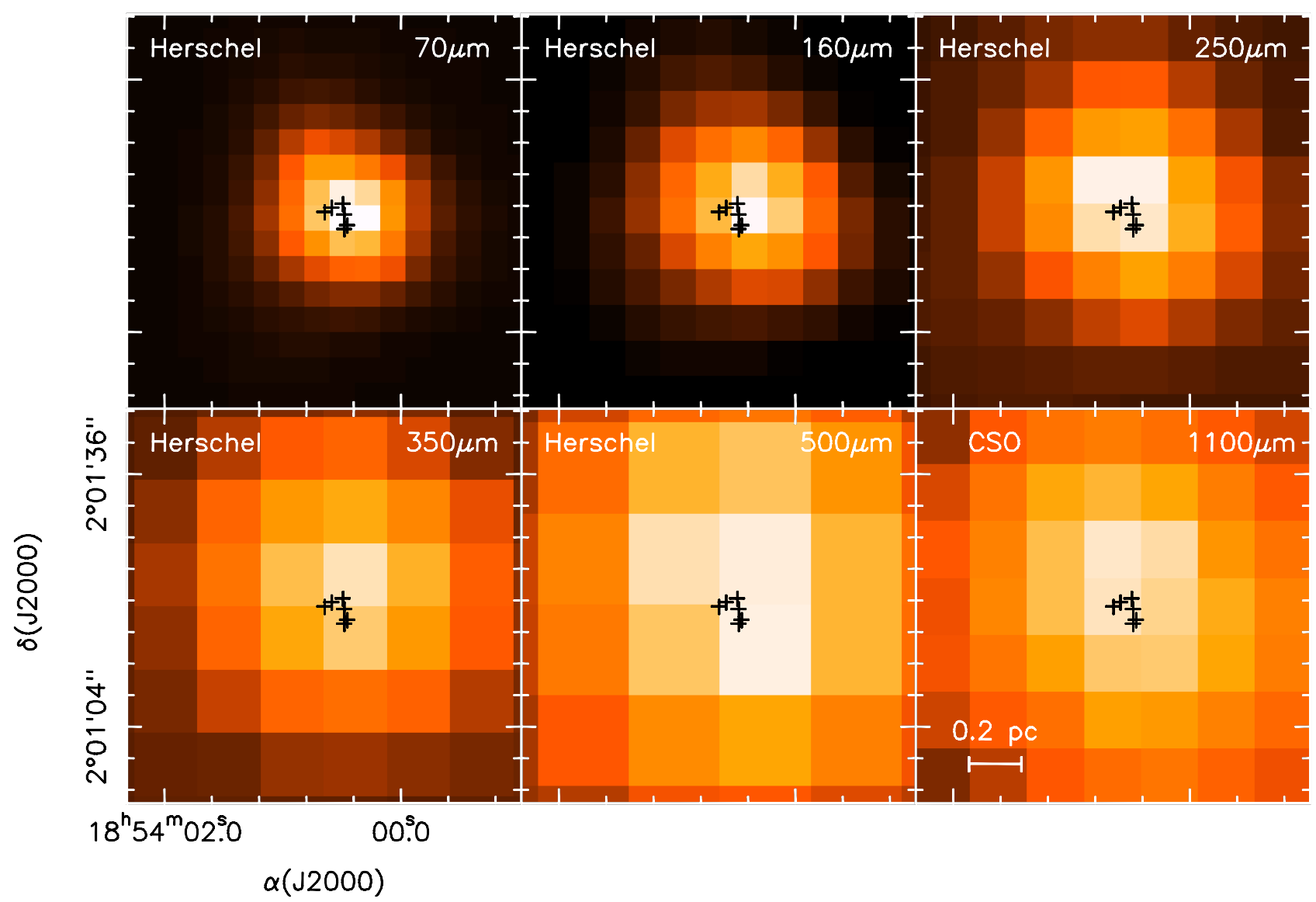

Fig. A.2. Herschel 70, 160, 250, 350, and $500 \mu \mathrm{m}$ (from the Hi-GAL survey), and CSO $1100 \mu \mathrm{m}$ (from the Bolocam survey) images on linear scale toward the star-forming region G35.03. The crosses indicate the positions of the submillimeter continuum cores (Table 3). 
A\&A 571, A52 (2014)

Appendix B: $\mathrm{CH}_{3} \mathrm{OH}$ myXCLASS fits

In Figs. B.1-B.6, we show the spectra of the methanol lines observed toward cores A, B, C, D, E, and F. The observed spectra (black lines) have been fitted (red lines) using myXCLASS (see Sect. 4.1). The procedure searches, by minimizing the $\chi^{2}$, the best fit of five input parameters: size (in $\operatorname{arcsec}$ ), temperature (in K), column density (in $\mathrm{cm}^{-2}$ ), line width (in $\mathrm{km} \mathrm{s}^{-1}$ ), and LSR velocity (in $\mathrm{km} \mathrm{s}^{-1}$ ). In Table 5, we list the values of the temperature, column density, and line width obtained in the best fits.

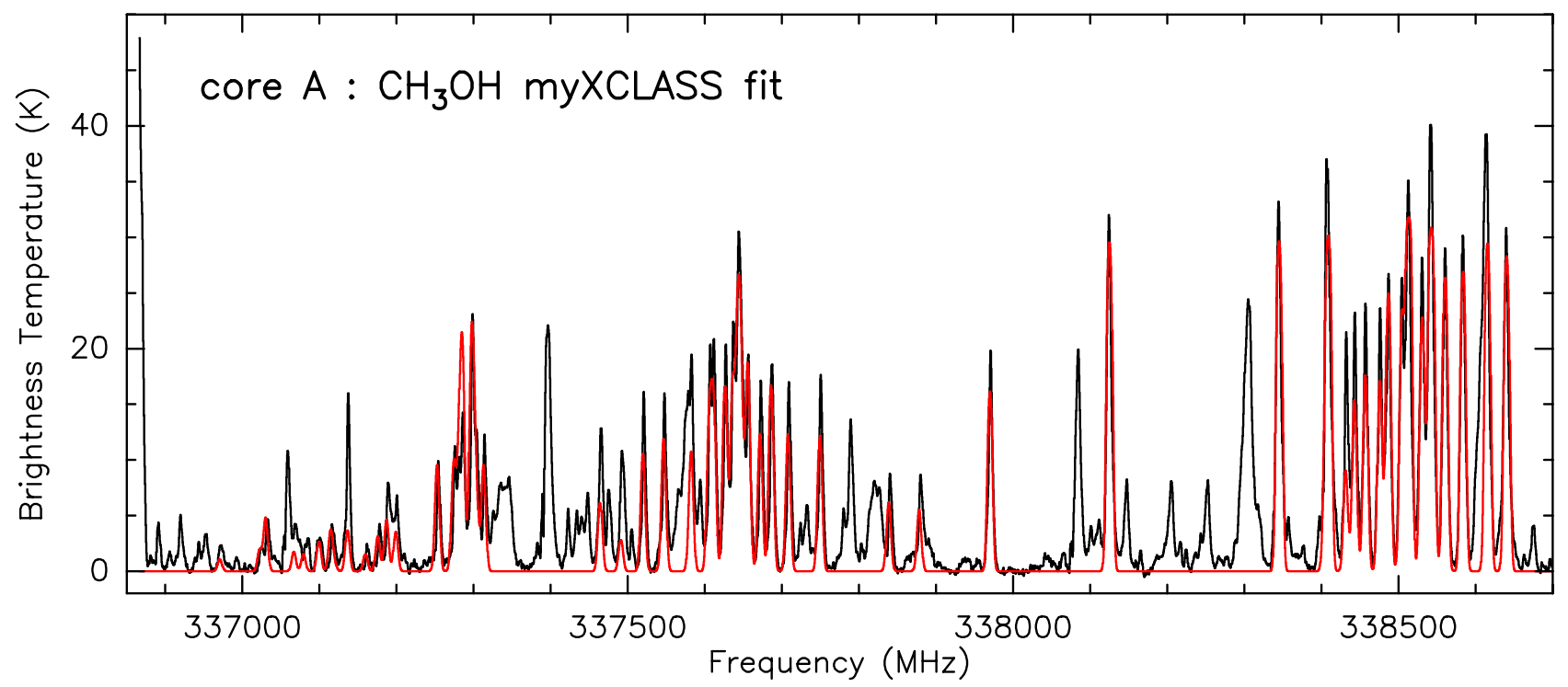

Fig. B.1. Observed $\mathrm{CH}_{3} \mathrm{OH}$ lines (black lines) toward core A in G35.03. The red line shows the best fit obtained with myXCLASS (see Sect. 4.1). The fit includes ground-state $\mathrm{CH}_{3} \mathrm{OH}$ and ${ }^{13} \mathrm{CH}_{3} \mathrm{OH}$ and torsionally excited $\mathrm{CH}_{3} \mathrm{OH}$ lines in the frequency ranges shown in the panel.

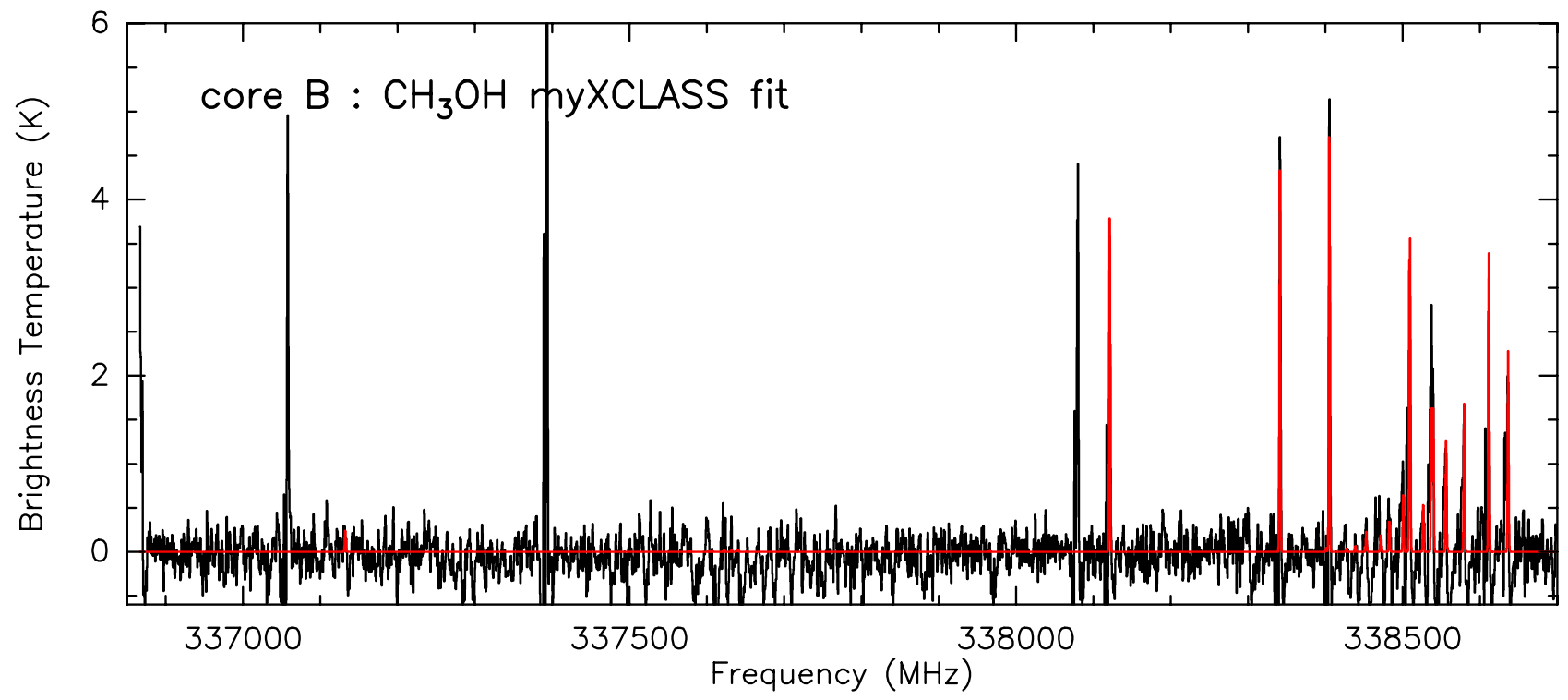

Fig. B.2. Same as Fig. B.1 for core B.

A52, page 22 of 24 
M. T. Beltrán et al.: The high-mass star-forming region G35.03+0.35 imaged with ALMA

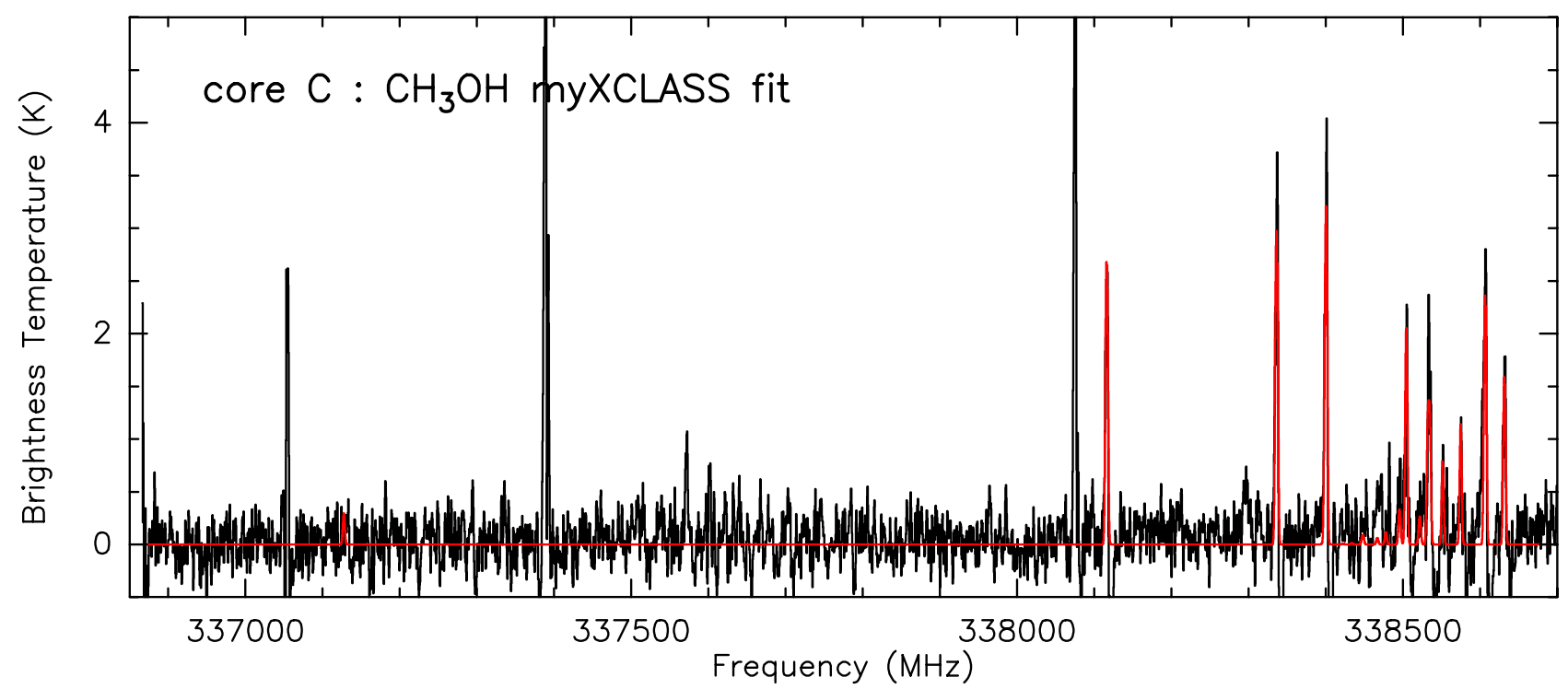

Fig. B.3. Same as Fig. B.1 for core C.

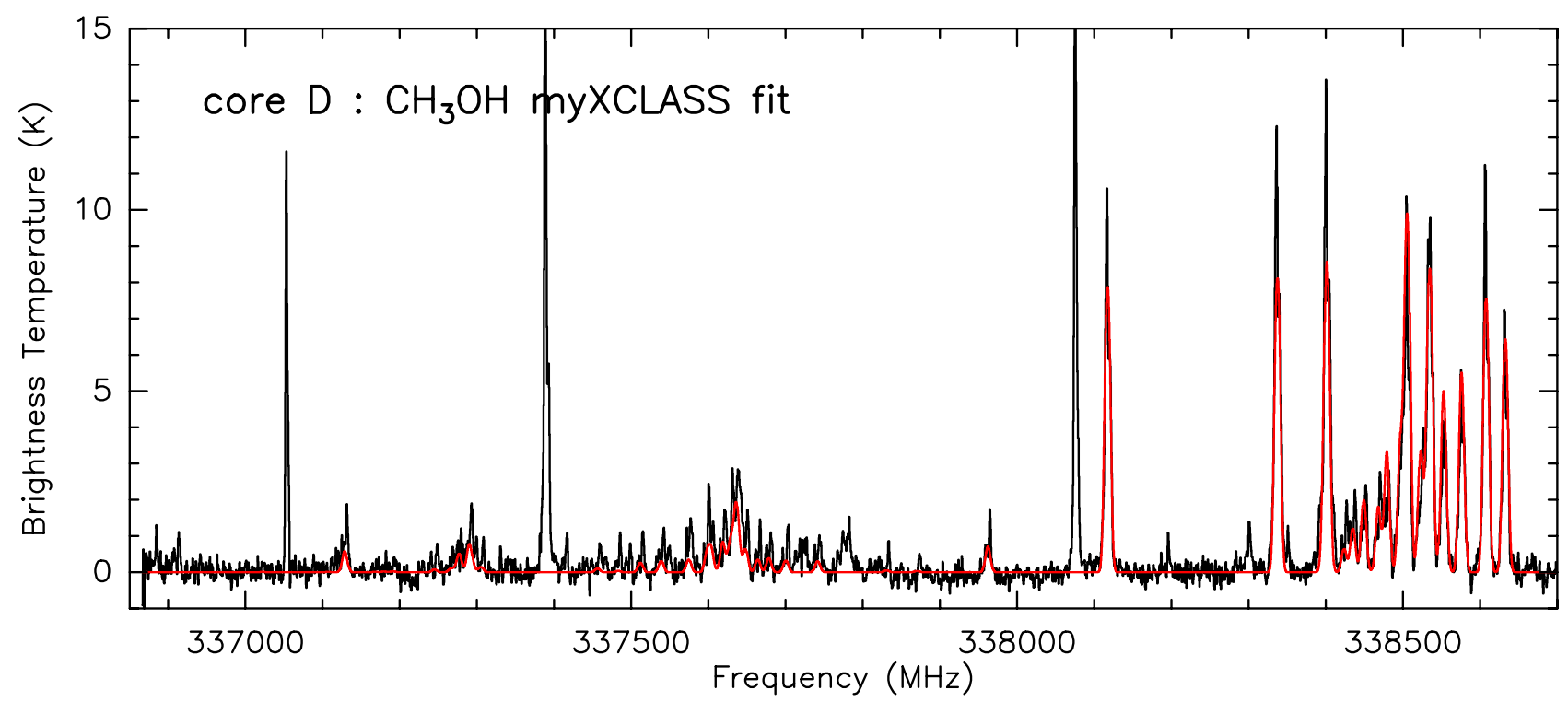

Fig. B.4. Same as Fig. B.1 for core D. 
A\&A 571, A52 (2014)

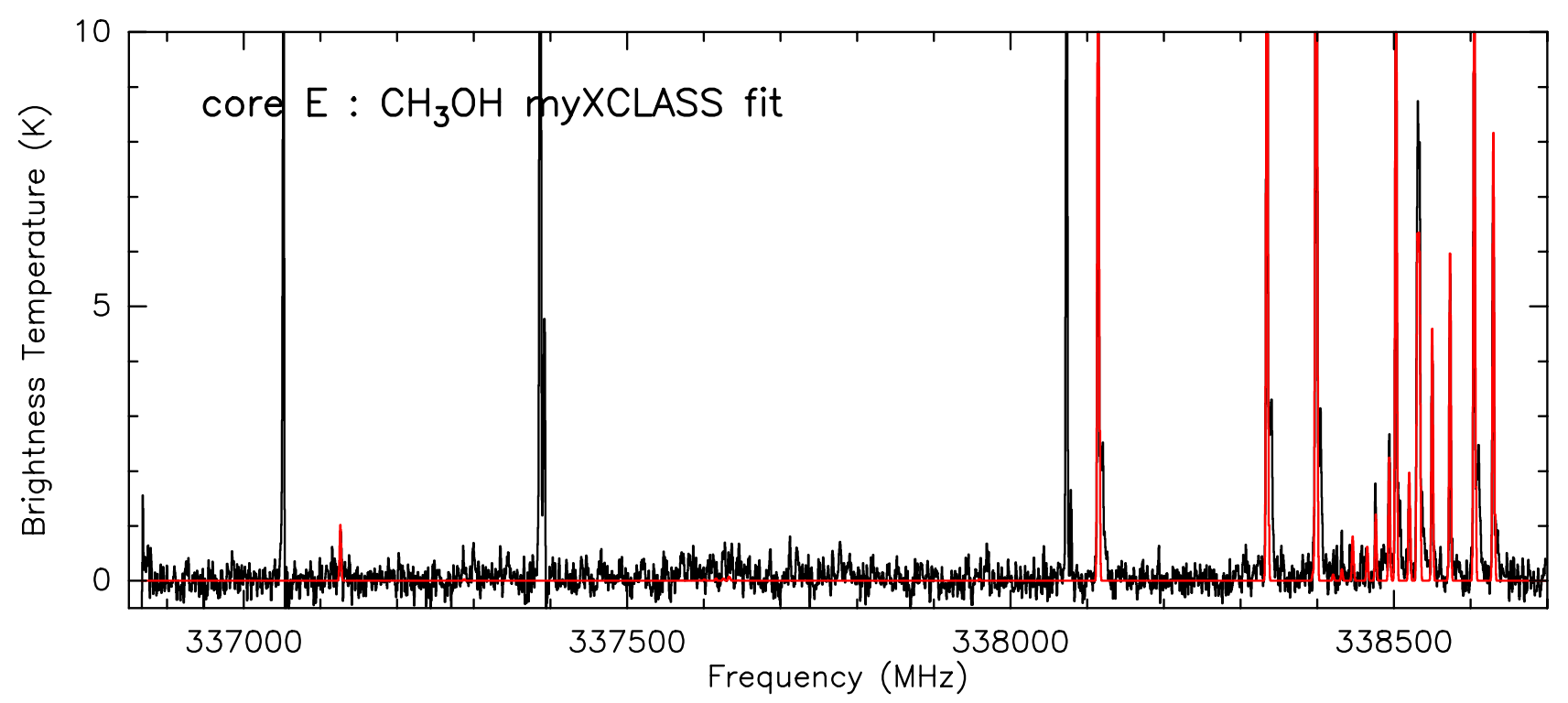

Fig. B.5. Same as Fig. B.1 for core E.

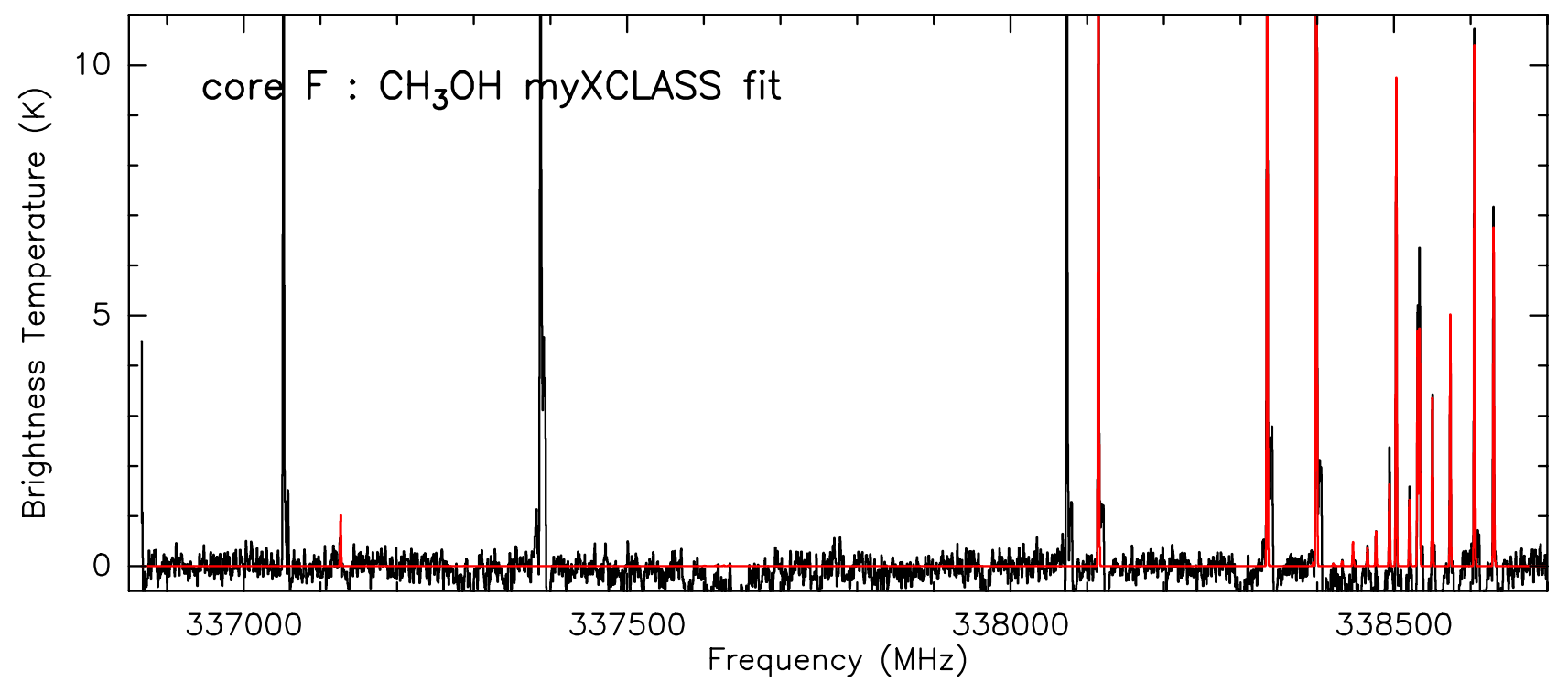

Fig. B.6. Same as Fig. B.1 for core F. 